\title{
The electroweak sector of the NMSSM at the one-loop level
}

\author{
Florian Staub, ${ }^{a}$ Werner Porod ${ }^{a, b}$ and Björn Herrmann ${ }^{c, a}$ \\ ${ }^{a}$ Institut für Theoretische Physik und Astrophysik, Universität Würzburg, \\ D-97074 Würzburg, Germany \\ ${ }^{b}$ AHEP Group, Institut de Física Corpuscular - C.S.I.C., \\ Universitat de València, E-46071 València, Spain \\ ${ }^{c}$ Deutsches Elektronen-Synchrotron (DESY), Theory group, \\ Notkestraße 85, D-22603 Hamburg, Germany \\ E-mail: florian.staub@physik.uni-wuerzburg.de, \\ porod@physik.uni-wuerzburg.de, bjoern.herrmann@desy.de
}

ABSTRACT: We present the electroweak spectrum for the Next-to-Minimal Supersymmetric Standard Model at the one-loop level, e.g. the masses of Higgs bosons, sleptons, charginos and neutralinos. For the numerical evaluation we present a mSUGRA variant with nonuniversal Higgs mass parameters squared and we compare our results with existing ones in the literature. Moreover, we briefly discuss the implications of our results for the calculation of the relic density.

Keywords: Supersymmetry Phenomenology

ARXIV EPRINT: 1007.4049 


\section{Contents}

1 Introduction 1

2 Calculation of the one-loop mass spectrum 2

2.1 Superpotential and soft SUSY breaking terms of the NMSSM 2

2.2 Minimum conditions of the vacuum 3

2.3 Masses of the Higgs bosons 4

2.4 Chargino and neutralino masses 5

2.5 Masses of sleptons 6

$\begin{array}{lll}3 & \text { The constrained NMSSM } & 7\end{array}$

$\begin{array}{lll}3.1 & \text { The model and its free parameters } & 7\end{array}$

3.2 Procedure to evaluate the SUSY parameters at the electroweak scale 8

$\begin{array}{lll}3.3 & \text { An example spectrum } & 8\end{array}$

4 Comparison with the literature $\quad 11$

$\begin{array}{ll}4.1 \text { Differences between the programs } & 12\end{array}$

$\begin{array}{lll}4.2 & \text { Results of the comparison } & 13\end{array}$

5 Effects on the relic density of dark matter 13

6 Conclusion $\quad 16$

$\begin{array}{ll}\text { A Squark mass matrices } & 17\end{array}$

$\begin{array}{ll}\text { B Anomalous dimensions } & 18\end{array}$

$\begin{array}{ll}\text { C Couplings } & 18\end{array}$

$\begin{array}{ll}\text { C.1 Two fermion - one scalar } & 19\end{array}$

C.2 Two fermion - one vector boson interaction 21

C.3 Two scalar - one vector boson interaction 23

C.4 One scalar - two vector boson — interaction 23

C.5 Two scalar - two vector boson interaction 24

C.6 Four scalar 24

$\begin{array}{lll}\text { C.7 Three scalar } & 30\end{array}$

$\begin{array}{ll}\text { D One-loop tadpoles } & 33\end{array}$

E One-loop self-energies $\quad 33$

E.1 Self energy of $Z$-boson 33

E.2 Self-energy of CP-even Higgs-bosons 34

E.3 Self-energy of CP-odd Higgs-bosons 36

E.4 Self-energy of the charged Higgs-boson 38 
$\begin{array}{lll}\text { E.5 Self-energy of neutralinos } & 40\end{array}$

E.6 Self-energy of charginos $\quad 42$

E.7 Self-energy of sleptons 43

E.8 Self-energy of sneutrinos 45

\section{Introduction}

Supersymmetric extensions of the standard model (SM) are promising candidates for new physics at the TeV scale $[1,3,4]$ as the solve several short-comings of the Standard Model (SM). The Minimal Supersymmetric Standard Model (MSSM) solves the hierarchy problem of the SM [5], leads to a unification of the gauge couplings [6-10] and introduces several candidates for dark matter depending on how SUSY is broken $[11,12]$. On the other hand, a new problem arises in the MSSM: the superpotential contains a parameter with mass dimension, namely the so called $\mu$ parameter which gives mass to the Higgs bosons and higgsinos. From a purely theoretical point of view, the value of this parameter is expected to be either of the order of the GUT/Planck scale or exactly zero, if it is protected by a symmetry. For phenomenological aspects, however, it is necessary that it is of the order of the scale of electroweak symmetry breaking (EWSB) and it has to be non-zero to be consistent with experimental data. This discrepancy is the so called $\mu$-problem of the MSSM [13].

The Next-to-Minimal Supersymmetric Standard Model (NMSSM) [14-27] provides an elegant solution to this problem. The particle content of the MSSM is extended by an additional gauge singlet, which receives a vacuum expectation value when supersymmetry is broken. The corresponding term in the superpotential gives then rise to an effective $\mu$-term which is naturally of the order of the EWSB scale. Also in this model several regions exist in parameter space where one obtains the correct relic density to explained the observed dark matter $[28,29]$. It turns out that in high scale models like mSUGRA several regions exist which are rather sensitive to mass differences of the various supersymmetric particles, in particular the masses of the Higgs bosons, the neutralinos and the staus, the supersymmetric partners of the tau-lepton, and require precise calculations of these masses. The corresponding regions are the so-called Higgs funnel(s) and the co-annihilation regions. Motivated by this observation we calculate the Higgs masses, the neutralino masses and the staus at the one-loop level.

This paper is organized as follows. We first detail our calculation of the mass spectrum in section 2. In section 3 we present the constrained NMSSM, which serves as our reference scenario and perform a numerical analysis of our implementation. section 4 is devoted to a comparison of our results with the public program package NMSSM-Tools [30-32]. Finally, we give a few examples for the calculation of the dark matter relic density in section 5 and draw our conclusions in section 6 . We collect the couplings and one-loop self-energies in the appendix where we include for completeness also those for the $Z$-boson and neutral Higgs bosons which have already been given in ref. [33]. 


\section{Calculation of the one-loop mass spectrum}

In this section we fix our notation and discuss briefly the $\overline{\mathrm{DR}}$ renormalization of the relevant masses, where we follow closely ref. [34].

\subsection{Superpotential and soft SUSY breaking terms of the NMSSM}

As already stated above, the solution to the $\mu$-problem of the MSSM is the replacement of the bilinear $\mu$-term by a coupling between the Higgs superfields and an additional gauge singlet $\hat{S}$ leading to the superpotential

$$
W_{\mathrm{NMSSM}}=-\hat{H}_{u} \hat{Q} Y_{u} \hat{U}^{c}+\hat{H}_{d} \hat{Q} Y_{d} \hat{D}^{c}+\hat{H}_{d} \hat{L} Y_{e} \hat{E}^{c}+\lambda \hat{H}_{u} \hat{H}_{d} \hat{S}+\frac{1}{3} \kappa \hat{S} \hat{S} \hat{S}
$$

where the last term is introduced to forbid a Peccei-Quinn symmetry which would lead to an axion in contradiction to experimental results, see e.g. ref. [35] and refs. therein. Moreover, we have only taken into account dimensionless couplings to avoid the $\mu$-problem of the MSSM.

The scalar component $S$ of $\hat{S}$ receives after SUSY breaking a vacuum expectation value (VEV), denoted $v_{s}$, which leads to

$$
\mu_{\mathrm{eff}}=\frac{1}{\sqrt{2}} \lambda v_{s}
$$

where we have used the decomposition

$$
S=\frac{1}{\sqrt{2}}\left(\phi_{s}+i \sigma_{s}+v_{s}\right) .
$$

Since $v_{s}$ and thus also $\mu_{\text {eff }}$ are a consequence of SUSY breaking one finds that $\mu_{\text {eff }}$ is naturally of the order of the SUSY breaking scale.

All interactions are fixed by the gauge structure and the above superpotential. We have used the Mathemtica package SARAH [36-38] to calculate all vertices, mass matrices including the one-loop corrections and renormalization group equations of the model.

In the following, we use the standard conventions, where for a matter superfield $\hat{X}$, $\tilde{X}$ denotes its scalar component and $X$ denotes its fermionic component. In case of the Higgs fields and the gauge singlet, $H_{u, d} / S$ are the scalar components, while $\tilde{H}_{u, d} / \tilde{S}$ are the fermionic higgsinos and the singlino.

At tree level the scalar potential receives contributions from several sources: from the the superpotential in eq. (2.1) the so-called $F$-terms given by

$$
\nu_{\mathrm{F}}=\sum_{i}\left|\frac{\partial W\left(\phi_{j}\right)}{\partial \phi_{i}}\right| .
$$

The sum runs over all chiral superfields $\hat{\phi}_{i}$, which are then replaced by their scalar component $\phi_{j}$. The $D$-terms are

$$
\mathcal{V}_{\mathrm{D}}=\frac{1}{2} \sum_{g} \sum_{a}\left|\sum_{i, j} \phi_{i}^{*} T_{g}^{a} \phi_{j}\right|^{2}
$$


and finally the soft breaking terms

$$
\begin{aligned}
\mathcal{V}_{\mathrm{SB}, 2}= & m_{H_{u}}^{2}\left|H_{u}\right|^{2}+m_{H_{d}}^{2}\left|H_{d}\right|^{2}+m_{S}^{2}|S|^{2}+\tilde{Q}^{\dagger} m_{\tilde{Q}}^{2} \tilde{Q}+ \\
& +\tilde{L}^{\dagger} m_{\tilde{L}}^{2} \tilde{L}+\tilde{D}^{\dagger} m_{\tilde{D}}^{2} \tilde{D}+\tilde{U}^{\dagger} m_{\tilde{U}}^{2} \tilde{U}+ \\
& +\frac{1}{2}\left(M_{1} \tilde{B} \tilde{B}+M_{2} \tilde{W}_{a} \tilde{W}^{a}+M_{3} \tilde{g}_{\alpha} \tilde{g}^{\alpha}+\text { h.c. }\right) \\
\mathcal{V}_{\mathrm{SB}, 3}= & -H_{u} \tilde{Q} T_{u} \tilde{U}^{\dagger}+H_{d} \tilde{Q} T_{d} \tilde{D}^{\dagger}+H_{d} \tilde{L} T_{e} \tilde{E}^{\dagger}+T_{\lambda} H_{u} H_{d} S+\frac{1}{3} T_{\kappa} S S S
\end{aligned}
$$

The sum in eq. (2.5) runs over all gauge groups $g$ and over the corresponding generators $a$, i.e. $\frac{1}{2} \lambda^{a}$ in the case of $\mathrm{SU}(3), \frac{1}{2} \sigma^{a}$ in the case of $\mathrm{SU}(2)$, and $\frac{3}{5} Y^{2}$ for the $\mathrm{U}(1)$. Here, $\lambda^{a}$ are the Gell-Mann matrices, $\sigma^{a}$ the Pauli-matrices, and $Y$ is the hypercharge.

\subsection{Minimum conditions of the vacuum}

Once electroweak symmetry gets broken, both Higgs doublets receive a VEV and we decompose the scalars similar to eq. (2.3)

$$
H_{u, d}=\frac{1}{\sqrt{2}}\left(\phi_{u, d}+i \sigma_{u, d}+v_{u, d}\right) .
$$

At tree level, the minimum conditions for the vacuum are the so-called tadpole equations

$$
T_{i}=\left.\frac{\partial V}{\partial v_{i}}\right|_{\phi_{i}=0, \sigma_{i}=0}=0
$$

with

$$
\begin{aligned}
T_{d}=\frac{\partial V}{\partial v_{d}}= & m_{H_{d}}^{2} v_{d}+\frac{1}{8} v_{d}\left(v_{d}^{2}-v_{u}^{2}\right)\left(g_{1}^{2}+g_{2}^{2}\right)+\frac{1}{2} v_{d}\left(v_{u}^{2}+v_{s}^{2}\right)|\lambda|^{2} \\
& -\frac{1}{2} v_{s}^{2} v_{u} \operatorname{Re}\{\kappa \lambda\}-\frac{1}{\sqrt{2}} v_{s} v_{u} \operatorname{Re}\left\{T_{\lambda}\right\}, \\
T_{u}=\frac{\partial V}{\partial v_{u}}= & m_{H_{u}}^{2} v_{d}+\frac{1}{8} v_{u}\left(v_{u}^{2}-v_{d}^{2}\right)\left(g_{1}^{2}+g_{2}^{2}\right)+\frac{1}{2} v_{u}\left(v_{d}^{2}+v_{s}^{2}\right)|\lambda|^{2} \\
& -\frac{1}{2} v_{s}^{2} v_{d} \operatorname{Re}\{\kappa \lambda\}-\frac{1}{\sqrt{2}} v_{s} v_{d} \operatorname{Re}\left\{T_{\lambda}\right\}, \\
T_{s}=\frac{\partial V}{\partial v_{s}}= & m_{S}^{2} v_{s}+v_{s}^{3}|\kappa|^{2}-v_{d} v_{s} v_{u} \operatorname{Re}\{\kappa \lambda\}+\frac{1}{2}\left(v_{d}^{2}+v_{u}^{2}\right) v_{s}|\lambda|^{2} \\
& +\frac{1}{\sqrt{2}} v_{s}^{2} \operatorname{Re}\left\{T_{\kappa}\right\}-\frac{1}{\sqrt{2}} v_{d} v_{u} \operatorname{Re}\left\{T_{\lambda}\right\} .
\end{aligned}
$$

Here we have chosen a phase convention where all VEVs are real. For the later calculation of the one-loop corrections to the Higgs boson masses one needs the evaluation of the tadpole equations at the one-loop level, leading to corrections $\delta t_{i}^{(1)}$. As renormalization condition we demand

$$
T_{i}+\delta t_{i}^{(1)}=0 \quad \text { for } \quad i=d, u, s .
$$

All calculations are performed in 't Hooft gauge using the diagrammatic approach. The explicit formulas for $\delta t_{i}^{(1)}$ are given in appendix D. In our subsequent analysis we will solve eqs. (2.13) for the soft SUSY breaking masses squared: $m_{H_{d}}^{2}, m_{H_{u}}^{2}$, and $m_{S}^{2}$. 
All parameters in eqs. (2.10)-(2.12) are understood as running parameters at a given renormalization scale $Q$. Note that the VEVs $v_{d}$ and $v_{u}$ are obtained from the running mass $m_{Z}(Q)$ of the $Z$-boson, which is related to the pole mass $m_{Z}$ through

$$
m_{Z}^{2}(Q)=\frac{g_{1}^{2}+g_{2}^{2}}{4}\left(v_{u}^{2}+v_{d}^{2}\right)=m_{Z}^{2}+\operatorname{Re}\left\{\Pi_{Z Z}^{T}\left(m_{Z}^{2}\right)\right\} .
$$

The transverse self-energy $\Pi_{Z Z}^{T}$ is given in appendix E.1. Details on the calculation of the running gauge couplings at $Q=m_{Z}$ can be found in ref. [34]. The ratio of these VEVs is denoted as in the MSSM by $\tan \beta=v_{u} / v_{d}$.

\subsection{Masses of the Higgs bosons}

The tree-level mass matrices for the neutral scalar Higgs bosons and pseudo scalar Higgs bosons can be calculated from the scalar potential according to

$$
m_{T, i j}^{2, h}=\left.\frac{\partial^{2} V}{\partial \phi_{i} \partial \phi_{j}}\right|_{\phi_{k}=0, \sigma_{k}=0}, \quad m_{T, i j}^{2, A^{0}}=\left.\frac{\partial^{2} V}{\partial \sigma_{i} \partial \sigma_{j}}\right|_{\phi_{k}=0, \sigma_{k}=0},
$$

respectively, with $i, j=1,2,3=u, d, s$. The matrices are symmetric and the entries in case of the scalar Higgs bosons are

$$
\begin{aligned}
& m_{T, 11}^{2, h}=m_{H_{d}}^{2}+\frac{1}{2}\left(v_{u}^{2}+v_{s}^{2}\right)|\lambda|^{2}+\frac{1}{8}\left(g_{1}^{2}+g_{2}^{2}\right)\left(3 v_{d}^{2}-v_{u}^{2}\right), \\
& m_{T, 12}^{2, h}=-\frac{1}{\sqrt{2}} v_{s} \operatorname{Re}\left\{T_{\lambda}\right\}-\frac{1}{2} v_{s}^{2} \operatorname{Re}\{\kappa \lambda\}-\frac{1}{4}\left(g_{1}^{2}+g_{2}^{2}-4|\lambda|^{2}\right) v_{d} v_{u}, \\
& m_{T, 13}^{2, h}=-\frac{1}{\sqrt{2}} v_{u} \operatorname{Re}\left\{T_{\lambda}\right\}+v_{s} v_{d}|\lambda|^{2}-v_{s} v_{u} \operatorname{Re}\{\kappa\}, \\
& m_{T, 22}^{2, h}=m_{H_{u}}^{2}+\frac{1}{2}\left(v_{d}^{2}+v_{s}^{2}\right)|\lambda|^{2}+\frac{1}{8}\left(g_{1}^{2}+g_{2}^{2}\right)\left(3 v_{u}^{2}-v_{d}^{2}\right), \\
& m_{T, 23}^{2, h}=-\frac{1}{\sqrt{2}} v_{d} \operatorname{Re}\left\{T_{\lambda}\right\}+v_{s} v_{u}|\lambda|^{2}-v_{s} v_{d} \operatorname{Re}\{\lambda \kappa\}, \\
& m_{T, 33}^{2, h}=m_{S}^{2}+3 v_{s}^{2}|\kappa|^{2}+\frac{1}{2}\left(v_{d}^{2}+v_{u}^{2}\right)|\lambda|^{2}+\sqrt{2} v_{s} \operatorname{Re}\left\{T_{\kappa}\right\}-v_{d} v_{u} \operatorname{Re}\{\kappa \lambda\},
\end{aligned}
$$

while those of the pseudo-scalar ones are

$$
\begin{aligned}
& m_{T, 11}^{2, A^{0}}=m_{H_{d}}^{2}+\frac{1}{2}\left(v_{u}^{2}+v_{s}^{2}\right)|\lambda|^{2}+\frac{1}{8}\left(g_{1}^{2}+g_{2}^{2}\right)\left(v_{d}^{2}-v_{u}^{2}\right), \\
& m_{T, 12}^{2, A^{0}}=\frac{1}{\sqrt{2}} v_{s} \operatorname{Re}\left\{T_{\lambda}\right\}+\frac{1}{2} v_{s}^{2} \operatorname{Re}\{\kappa \lambda\}, \\
& m_{T, 13}^{2, A^{0}}=\frac{1}{\sqrt{2}} v_{u} \operatorname{Re}\left\{T_{\lambda}\right\}-v_{s} v_{u} \operatorname{Re}\{\kappa \lambda\}, \\
& m_{T, 22}^{2, A^{0}}=m_{H_{u}}^{2}+\frac{1}{2}\left(v_{d}^{2}+v_{s}^{2}\right)|\lambda|^{2}+\frac{1}{8}\left(g_{1}^{2}+g_{2}^{2}\right)\left(v_{u}^{2}-v_{d}^{2}\right), \\
& m_{T, 23}^{2, A^{0}}=\frac{1}{\sqrt{2}} v_{d} \operatorname{Re}\left\{T_{\lambda}\right\}-v_{d} v_{s} \operatorname{Re}\{\kappa \lambda\}, \\
& m_{T, 33}^{2, A^{0}}=m_{S}^{2}+v_{s}^{2} \kappa^{2}+\frac{1}{2}\left(v_{d}^{2}+v_{u}^{2}\right)|\lambda|^{2}-\sqrt{2} v_{s} \operatorname{Re}\left\{T_{\kappa}\right\}+v_{d} v_{u} \operatorname{Re}\{\kappa \lambda\},
\end{aligned}
$$

where $m_{H_{d}}^{2}, m_{H_{u}}^{2}$ and $m_{S}^{2}$ satisfy the tadpole equations. 
The diagonalization of the mass matrices $m_{T}^{2, h}$ and $m_{T}^{2, A^{0}}$ leads in total to five physical mass eigenstates and one neutral Goldstone boson which becomes the longitudinal component of the Z-boson. The five physical degrees of freedom are: three CP-even Higgs bosons denoted $h_{1,2,3}$ and two CP-odd bosons denoted $A_{1,2}^{0}$. The corresponding rotation matrices $Z^{h}$ und $Z^{A^{0}}$ are defined through

$$
Z^{x} m^{2, x} Z^{x, T}=m_{\text {diag }}^{2, x}, \quad x=h, A^{0} .
$$

Moreover, we note that we order all masses such, that $m_{i} \leq m_{j}$ if $i<j$.

The one-loop scalar Higgs masses are then calculated by taking the real part of the poles of the corresponding propagator matrices

$$
\operatorname{Det}\left[p_{i}^{2} \mathbf{1}-m_{1 L}^{2, h}\left(p^{2}\right)\right]=0
$$

where

$$
m_{1 L}^{2, h}\left(p^{2}\right)=\tilde{m}_{T}^{2, h}-\Pi_{h h}\left(p^{2}\right) .
$$

Here, $\tilde{m}_{h}$ is the tree-level mass matrix from eq. (2.15). Equation (2.29) has to be solved for each eigenvalue $p^{2}=m_{i}^{2}$. The same procedure is also applied for the pseudo scalar Higgs bosons. The complete 1-loop expressions for the self energy of the CP-odd and even Higgs bosons are given in appendices E.2 and E.3.

The charged Higgs sector consists of $H_{d}^{-}$and $H_{u}^{+}$. The mass matrix in the basis $\left(H_{d}^{-}, H_{u}^{+, *}\right)$ is diagonalized by a unitary matrix $Z^{+}$

$$
Z^{+} m^{2, H^{+}} Z^{+, \dagger}=m_{\text {diag }}^{2, H^{+}} .
$$

The eigenstates yield as in the MSSM the longitudinal component of the $W$-boson and a charged Higgs boson $H^{+}$with mass

$$
m_{T}^{2, H^{+}}=\frac{\left(v_{d}^{2}+v_{u}^{2}\right)\left(2 v_{s} \operatorname{Re}\{\kappa \lambda\}+v_{d} v_{u}\left(g_{2}^{2}-2|\lambda|^{2}\right)+2 \sqrt{2} v_{s} \operatorname{Re}\left\{T_{\lambda}\right\}\right)}{4 v_{d} v_{u}}
$$

and the one-loop mass

$$
m_{1 L}^{2, H^{+}}=m_{T}^{2, H^{+}}-\operatorname{Re}\left\{\Pi_{H^{+} H^{+}}\left(m_{1 L}^{2, H^{+}}\right)\right\}
$$

where the self-energy can be found in appendix E.4

\subsection{Chargino and neutralino masses}

As for the Higgs bosons discussed in the previous section, one has to find the real parts of the poles of the propagator matrix to obtain the masses of charginos and neutralinos. At the tree-level the chargino mass matrix in the basis $\tilde{\psi}^{-}=\left(\tilde{W}^{-}, \tilde{H}_{d}^{-}\right)^{T}, \tilde{\psi}^{+}=\left(\tilde{W}^{+}, \tilde{H}_{u}^{+}\right)$is given by

$$
\mathcal{L}_{\tilde{\psi}^{+}}=-\tilde{\psi}^{-T} M_{T}^{\tilde{\chi}^{+}} \tilde{\psi}^{+}+\text {h.c. }
$$

with

$$
M_{T}^{\tilde{\chi}^{+}}=\left(\begin{array}{cc}
M_{2} & \frac{1}{\sqrt{2}} g_{2} v_{u} \\
\frac{1}{\sqrt{2}} g_{2} v_{d} & \frac{1}{\sqrt{2}} v_{s} \lambda
\end{array}\right) .
$$


This mass matrix is diagonalized by a biunitary transformation such that $U^{*} M_{T}^{\tilde{\chi}^{+}} V^{\dagger}$ is diagonal. The matrices $U$ and $V$ are obtained by diagonalizing $M_{T}^{\tilde{\chi}^{+}}\left(M_{T}^{\tilde{\chi}^{+}}\right)^{\dagger}$ and $\left(M_{T}^{\tilde{\chi}^{+}}\right)^{*}\left(M_{T}^{\tilde{\chi}^{+}}\right)^{T}$, respectively. At the one-loop level, one has to add the self-energies

$$
M_{1 L}^{\tilde{\chi}^{+}}\left(p_{i}^{2}\right)=M_{T}^{\tilde{\chi}^{+}}-\Sigma_{S}^{+}\left(p_{i}^{2}\right)-\Sigma_{R}^{+}\left(p_{i}^{2}\right) M_{T}^{\tilde{\chi}^{+}}-M_{T}^{\tilde{\chi}^{+}} \Sigma_{L}^{+}\left(p_{i}^{2}\right) .
$$

In case of the neutralinos one has a complex symmetric $5 \times 5$ mass matrix which in the basis $\tilde{\psi}^{0}=\left(\tilde{B}, \tilde{W}^{0}, \tilde{H}_{d}^{0}, \tilde{H}_{u}^{0}, \tilde{S}\right)^{T}$ is at the tree-level given by

$$
M_{T}^{\tilde{\chi}^{0}}=\left(\begin{array}{ccccc}
M_{1} & 0 & -\frac{1}{2} g_{1} v_{d} & \frac{1}{2} g_{1} v_{u} & 0 \\
0 & M_{2} & \frac{1}{2} g_{2} v_{d} & -\frac{1}{2} g_{2} v_{u} & 0 \\
-\frac{1}{2} g_{1} v_{d} & \frac{1}{2} g_{2} v_{d} & 0 & -\frac{1}{\sqrt{2}} v_{s} \lambda & -\frac{1}{\sqrt{2}} v_{u} \lambda \\
\frac{1}{2} g_{1} v_{u} & -\frac{1}{2} g_{2} v_{u} & -\frac{1}{\sqrt{2}} v_{s} \lambda & 0 & -\frac{1}{\sqrt{2}} v_{d} \lambda \\
0 & 0 & -\frac{1}{\sqrt{2}} v_{u} \lambda & -\frac{1}{\sqrt{2}} v_{d} \lambda & \sqrt{2} v_{s} \kappa
\end{array}\right) .
$$

One can show that for real parameters the matrix $M_{T}^{\tilde{\chi}^{0}}$ can be diagonalized directly by a $5 \times 5$ mixing matrix $N$ such that $N^{*} M_{T}^{\tilde{\chi}^{0}} N^{\dagger}$ is diagonal. In the complex case, one has to diagonalize $M_{T}^{\tilde{\chi}^{0}}\left(M_{T}^{\tilde{\chi}^{0}}\right)^{\dagger}$. At the one-loop level we obtain

$$
\begin{aligned}
M_{1 L}^{\tilde{\chi}^{0}}\left(p_{i}^{2}\right)=M_{T}^{\tilde{\chi}^{0}}-\frac{1}{2}[ & \Sigma_{S}^{0}\left(p_{i}^{2}\right)+\Sigma_{S}^{0, T}\left(p_{i}^{2}\right)+\left(\Sigma_{L}^{0, T}\left(p_{i}^{2}\right)+\Sigma_{R}^{0}\left(p_{i}^{2}\right)\right) M_{T}^{\tilde{\chi}^{0}} \\
& \left.+M_{T}^{\tilde{\chi}^{0}}\left(\Sigma_{R}^{0, T}\left(p_{i}^{2}\right)+\Sigma_{L}^{0}\left(p_{i}^{2}\right)\right)\right] .
\end{aligned}
$$

The complete self-energies for neutralinos and charginos are given in appendices E.5 and E.6, respectively.

\subsection{Masses of sleptons}

In the basis $\left(\tilde{e}_{L}, \tilde{\mu}_{L}, \tilde{\tau}_{L}, \tilde{e}_{R}, \tilde{\mu}_{R}, \tilde{\tau}_{R}\right)$, the mass matrix of the charged sleptons at the tree-level is given by

$$
m_{T}^{2, \tilde{l}}=\left(\begin{array}{cc}
m_{L L}^{2} & -\frac{1}{2} v_{s} v_{u} \lambda^{*} Y_{e}^{T}+\frac{1}{\sqrt{2}} v_{d} T_{e}^{T} \\
-\frac{1}{2} v_{s} v_{u} \lambda Y_{e}^{*}+\frac{1}{\sqrt{2}} v_{d} T_{e}^{*} & m_{R R}^{2}
\end{array}\right)
$$

with the diagonal entries

$$
\begin{aligned}
& m_{L L}^{2}=m_{\tilde{L}}^{2}+\frac{v_{d}^{2}}{2}\left(Y_{e}\right)^{*}\left(Y_{e}\right)^{T}+\frac{1}{8}\left(g_{1}^{2}-g_{2}^{2}\right)\left(v_{d}^{2}-v_{u}^{2}\right) \mathbf{1}_{3}, \\
& m_{R R}^{2}=m_{\tilde{E}}^{2}+\frac{v_{d}^{2}}{2}\left(Y_{e}\right)^{T}\left(Y_{e}\right)^{*}+\frac{g_{1}^{2}}{4}\left(v_{u}^{2}-v_{d}^{2}\right) \mathbf{1}_{3} .
\end{aligned}
$$

Where $\mathbf{1}_{3}$ is the $3 \times 3$ unit matrix. This matrix is diagonalized by a unitary mixing matrix $Z^{E}$ :

$$
Z^{E} m_{T}^{2, \tilde{l}} Z^{E \dagger}=m_{\text {diag }}^{2, \tilde{l}}
$$

The corresponding mass matrix at the one-loop level is again obtained by taking into account the self-energy according to

$$
m_{1 L}^{2, \tilde{l}}\left(p_{i}^{2}\right)=m_{T}^{2, \tilde{l}}-\Pi_{\tilde{l} l}\left(p_{i}^{2}\right)
$$


and the one-loop masses are obtained by calculating the real parts of the poles of the propagator matrix. The expression for $\Pi_{\tilde{l}}\left(p_{i}^{2}\right)$ can be found in appendix E.7.

Finally, in the basis $\left(\tilde{\nu}_{e}, \tilde{\nu}_{\mu}, \tilde{\nu}_{\tau}\right)$ the tree-level sneutrino mass matrix is given by

$$
m_{T}^{2, \tilde{\nu}}=m_{\tilde{L}}^{2}+\frac{1}{8}\left(g_{1}^{2}+g_{2}^{2}\right)\left(v_{d}^{2}-v_{u}^{2}\right) \mathbf{1}_{3} .
$$

This matrix is diagonalized by a unitary mixing matrix $Z^{E}$ :

$$
Z^{\nu} m_{T}^{2, \tilde{\nu}} Z^{\nu \dagger}=m_{\text {diag }}^{2, \tilde{\nu}}
$$

Similarly as above the one loop mass matrix is given by

$$
m_{1 L}^{2, \tilde{\nu}}\left(p_{i}^{2}\right)=m_{T}^{2, \tilde{\nu}}-\Pi_{\tilde{\nu} \tilde{\nu}}\left(p_{i}^{2}\right) .
$$

The one-loop masses are obtained by calculating the real parts of the poles of the propagator matrix. The expression for $\Pi_{\tilde{\nu} \tilde{\nu}}\left(p_{i}^{2}\right)$ can be found in appendix E.8.

\section{The constrained NMSSM}

\subsection{The model and its free parameters}

In the subsequent numerical analysis, we are mainly interested in precision calculation of the SUSY masses and potential effects in the calculation of the relic density. To reduce the number of free parameters we therefore focus on a scenario motivated by minimal supergravity (mSUGRA) [39]. More precisely, we study a variant of the constrained NMSSM [40, 41] where we allow for non-universal Higgs mass parameters squared at the GUT scale. In our setup, these parameters are determined with the help of the tadpole equations (2.13) at the electroweak scale. As a side remark, we note that also other recently used mSUGRA versions of the NMSSM contained non-minimal features either for the scalar mass parameter and/or for the trilinear couplings.

We apply the following boundary conditions for the gaugino masses $M_{1}, M_{2}, M_{3}$ and the soft breaking masses of the squarks and sleptons $m_{i}^{2}$ at the GUT scale, which is defined as the scale where the $U_{Y}(1)$ and $\mathrm{SU}(2)_{L}$ couplings fulfill $\sqrt{\frac{5}{3}} g_{1}=g_{2}$ :

$$
\begin{array}{r}
M_{1}=M_{2}=M_{3} \equiv M_{1 / 2}, \\
m_{\tilde{D}}^{2}=m_{\tilde{U}}^{2}=m_{\tilde{Q}}^{2}=m_{\tilde{E}}^{2}=m_{\tilde{L}}^{2} \equiv m_{0}^{2} \mathbf{1}_{3} .
\end{array}
$$

The trilinear scalar couplings $T_{i}$ are given by

$$
T_{u}=A_{0} Y_{u}, \quad T_{d}=A_{0} Y_{d}, \quad T_{e}=A_{0} Y_{e}, \quad T_{\lambda}=A_{\lambda} \lambda, \quad \text { and } \quad T_{\kappa}=A_{\kappa} \kappa .
$$

Here, $A_{0}$ is defined at the GUT scale, while $\lambda, \kappa, A_{\lambda}$ and $A_{\kappa}$ can be defined either at the GUT or at the SUSY scale. Together with the values for $\tan \beta=\frac{v_{u}}{v_{d}}$ and $v_{s}$ the spectrum is fixed. To summarize, we have nine input parameters,

$$
M_{1 / 2}, \quad m_{0}, \quad A_{0}, \quad \lambda, \quad \kappa, \quad A_{\lambda}, \quad A_{\kappa}, \quad v_{s}, \quad \text { and } \tan \beta .
$$

Note, that we allow for non-universalities in the trilinear parameters for an easier comparison with the existing literature but in principal we could take all $A$-parameters equal at the GUT scale. We choose in the following $v_{s}>0$ and $\lambda, \kappa \in[-1,1]$. 


\subsection{Procedure to evaluate the SUSY parameters at the electroweak scale}

In order to connect the parameters at various scales, we use the renormalization group equations (RGEs), which are calculated at the two-loop level in the most general form using the generic formulas given in ref. [42]. We have compared the obtained expressions for the RGEs with those given in ref. [35] in the limit where only the third generation Yukawa couplings contributes. There has been a slight difference in the two-loop $\beta$-function of $A_{\lambda}=T_{\lambda} / \lambda$, but it was confirmed by the authors of ref. [35] that our result is correct. The RGEs themselves can easily be calculated by the CalcRGEs command of SARAH and a print-out can be found at [43].

In the calculation of the gauge and Yukawa couplings we follow closely the procedure described in ref. [44]: the values for the Yukawa couplings giving mass to the SM fermions and the gauge couplings are determined at the scale $M_{Z}$ based on the measured values for the quark, lepton and vector boson masses as well as for the gauge couplings. Here, we have included the one-loop corrections to the mass of W- and Z-boson as well as the SUSY contributions to $\delta_{V B}$ for calculating the gauge couplings. Similarly, we have included the complete one-loop corrections to the self-energies of SM fermions extending the formulas of [34] to include the additional neutralino and Higgs bosons. Moreover, we have resummed the $\tan \beta$ enhanced terms for the calculation of the Yukawa couplings of the $b$-quark and the $\tau$-lepton as in [44]. The vacuum expectation values $v_{d}$ and $v_{u}$ are calculated with respect to the given value of $\tan \beta$ at $M_{Z}$. Furthermore, we solve the tadpole equations to get initial values for $m_{H_{d}}^{2}, m_{H_{u}}^{2}$ and $m_{S}^{2}$. Afterwards the RGEs are used to obtain the values at the GUT scale and all boundary conditions including $\lambda$ and $\kappa$ are set as described above. Then, an RGE running to the SUSY scale is performed and the SUSY masses are calculated at the one-loop level and for the neutral and pseudo scalar Higgs bosons we include beside the one-loop contributions presented here also the known two-loop contributions [33]. For this purpose also the numerical the values for the VEVs at $M_{\text {SUSY }}$ are needed. These are derived using the two-loop RGEs

$$
\beta_{v_{i}}=-v_{i}\left(\gamma_{i}^{(1)}+\gamma_{i}^{(2)}\right)
$$

with $i=u, d$. Here, $\gamma_{i}^{(1)}$ and $\gamma_{i}^{(2)}$ are the anomalous dimensions for the two Higgs-doublets at the one- and two-loop level, respectively. The corresponding expressions are given in appendix B. Let us recall that the input value for $v_{s}$ is already given at $M_{\text {SUSY }}$. These steps are iterated until the masses converge with a relative precision of $10^{-5}$. The complete procedure has been implemented in SPheno [44]. ${ }^{1}$

\subsection{An example spectrum}

In table 1 we give as an example the masses of the Higgs bosons, chargino, neutralinos and third generation sfermions at tree-level as well as at the one- and two- loop level for the

\footnotetext{
${ }^{1}$ This special version can be obtained from the authors and will become public in the near future.
} 


\begin{tabular}{|c|c|c|c|c|c|}
\hline Particle & $m_{T}[\mathrm{GeV}]$ & $m_{1 L}[\mathrm{GeV}]$ & $\Delta[\%]$ & $m_{2 L}[\mathrm{GeV}]$ & $\Delta[\%]$ \\
\hline$h_{1}$ & 86.7 & 113.3 & 23.5 & 119.6 & 5.2 \\
$h_{2}$ & 863.1 & 934.2 & 7.6 & 937.3 & 0.3 \\
$h_{3}$ & 2073.9 & 2073.9 & $<0.1$ & 2073.9 & $<0.1$ \\
$A_{1}^{0}$ & 76.4 & 69.3 & 10.2 & 69.5 & 0.3 \\
$A_{2}^{0}$ & 865.2 & 937.2 & 7.7 & 940.4 & 0.3 \\
\hline$\tilde{\chi}_{1}^{0}$ & 211.6 & 207.6 & 1.9 & - & - \\
$\tilde{\chi}_{2}^{0}$ & 388.2 & 398.4 & 2.6 & - & - \\
$\tilde{\chi}_{3}^{0}$ & 987.9 & 980.5 & 0.7 & - & - \\
$\tilde{\chi}_{4}^{0}$ & 993.0 & 985.1 & 0.8 & - & - \\
$\tilde{\chi}_{5}^{0}$ & 2074.8 & 2074.9 & $<0.1$ & - & - \\
$\tilde{\chi}_{1}^{+}$ & 388.2 & 398.6 & 2.6 & - & - \\
$\tilde{\chi}_{2}^{+}$ & 993.3 & 985.9 & 0.7 & - & - \\
\hline$\tilde{\tau}_{1}$ & 191.1 & 193.3 & 1.2 & - & - \\
$\tilde{\tau}_{2}$ & 388.1 & 393.1 & 1.1 & - & - \\
$\tilde{t}_{1}$ & 506.9 & 541.8 & 6.4 & - & - \\
$\tilde{t}_{2}$ & 914.4 & 949.3 & 3.7 & - & - \\
$\tilde{b}_{1}$ & 845.3 & 880.4 & 3.9 & - & - \\
$\tilde{b}_{2}$ & 961.9 & 1008.5 & 4.6 & - & - \\
\hline$\tilde{g}$ & 1107.2 & 1154.2 & 4.1 & - & - \\
\hline
\end{tabular}

Table 1. Comparison of the tree-level $m_{T}$ and loop masses at 1-loop $\left(m_{1 L}\right)$ and 2-loop $\left(m_{2 L}\right) . \Delta$ is the relative difference $\left|1-\frac{m_{T}}{m_{1 L}}\right|$ respectively $\left|1-\frac{m_{1 L}}{m_{2 L}}\right|$.

parameter set

$$
\begin{array}{rlrlrl}
m_{0} & =180 \mathrm{GeV}, & m_{1 / 2} & =500 \mathrm{GeV}, & A_{0}=A_{\lambda}^{\mathrm{GUT}} & =-1500 \mathrm{GeV}, \\
\tan \beta & =10, & \kappa_{\kappa}^{\mathrm{GUT}} & =-36 \mathrm{GeV}, \\
& \lambda^{\mathrm{GUT}}=0.11, & v_{s} & =13689 \mathrm{GeV} .
\end{array}
$$

which is close to the benchmark scenario 1 of ref. [40]. As can be seen in table 1 , the corrections are sizable ranging from $0.1 \%$ to $23.6 \%$ in case of the lightest Higgs boson. This large correction is well known and the main reason for including the two-loop corrections. The corresponding two-loop Higgs masses as well as the relative correction with respect to the one-loop results are also displayed in table 1. Again the largest correction with $5.2 \%$ is in case of the lightest Higgs boson mass.

As an estimate of the remaining theoretical uncertainty we have varied the renormalization scale in SPheno. We show in figure 1 the scale dependence for masses of neutral scalar Higgs boson at the one- and two-loop masses normalized to their values at $Q=1$ $\mathrm{TeV}$ and vary the renormalization scale $Q$ between $200 \mathrm{GeV}$ and $2.2 \mathrm{TeV}$. As can be seen, the large variation of $8 \%$ at one-loop for the lightest Higgs, which is mainly the lighter $\mathrm{SU}(2)$ doublet Higgs in this case, is reduced at two-loop to less than $2 \%$. In case of the heavier Higgs bosons the scale dependence is significantly smaller showing a significant improvement when going from the one-loop level to the two-loop level. However, we remark 

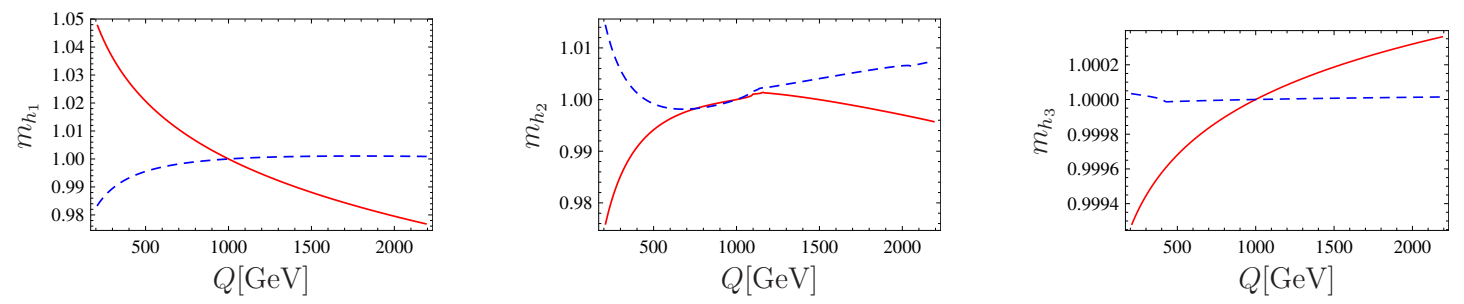

Figure 1. Dependence of CP even Higgs masses on the renormalization scale $Q$ at 1-loop (red) and 2-loop level (dashed blue) normalized to the value at $Q=1 \mathrm{TeV}$. From left to right: $m_{h_{1}}, m_{h_{2}}$ and $m_{h_{3}}$.
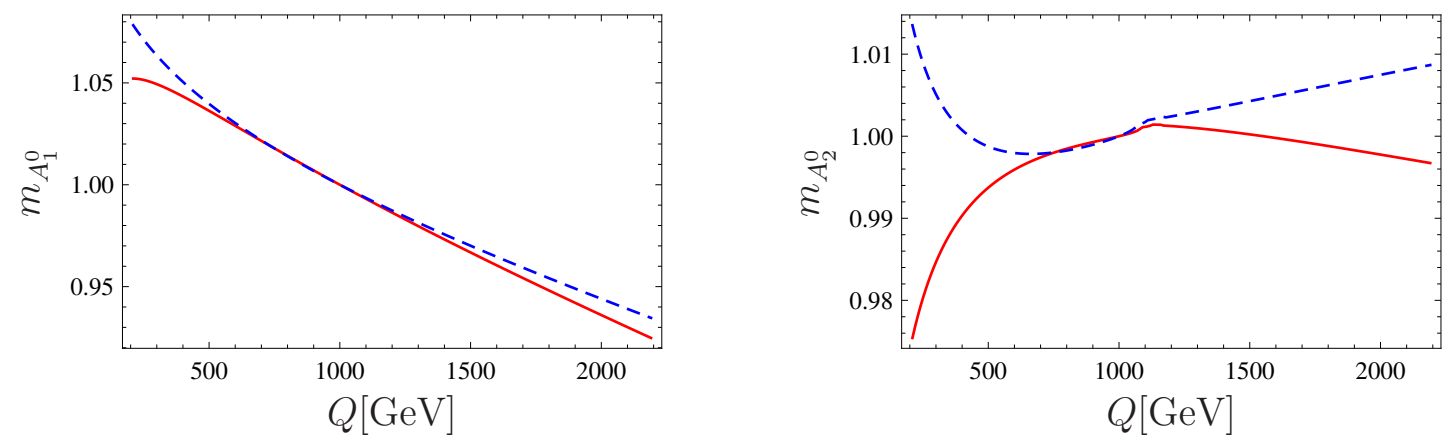

Figure 2. Dependence of CP odd Higgs masses on the renormalization scale $Q$ at 1-loop (red) and 2-loop level (dashed blue) normalized to the value at $Q=1 \mathrm{TeV}$. Left: $m_{A_{1}^{0}}$. Right: $m_{A_{2}^{0}}$.

that the values of $\lambda$ and $\kappa$ are small in this scenario and we expect a stronger dependence in case of larger couplings.

The picture changes slightly in the case of the pseudo scalar bosons as can be seen in figure 2. While the heavier pseudo scalar behaves exactly as the second scalar field since both originate to $99.5 \%$ from $H_{d}$, the scale dependence for the lighter pseudo scalar is smaller compared to the lightest scalar field, but hardly improves at the two-loop level. This is because in the two-loop part contain 'only' the strong contributions of the third generation squarks whereas this state is mainly a singlet state and, thus, the contributions due to the Yukawa couplings would be needed for a further improvement.

In figure 3 the scale dependence for different neutralinos is shown. As can be seen, in case of the three lighter states the scale dependence is reduced from the level of about $1.5 \%$ to 3-5 per-mill. In case of the singlet state $\tilde{\chi}_{5}$ the scale dependence is already small due to the small values of $\lambda$ and $\kappa$. We note that the scale dependence of $\tilde{\chi}_{1}^{+}\left(\tilde{\chi}_{2}^{+}\right.$and $\left.\tilde{\chi}_{4}^{0}\right)$ is nearly the same as that of $\tilde{\chi}_{2}^{0}\left(\tilde{\chi}_{3}^{0}\right)$ as these state have their main origin in the same electroweak multiplet.

Finally we show in figure 4 the scale dependence of the staus. The scale dependence at tree level amounts to about $2-2.5 \%$ and is reduced at one-loop level to about $1 \%$ and less where the $\tilde{\tau}_{1}$ shows still the larger dependence. The sleptons of the first two generations show a somewhat smaller scale dependence as in their cases the Yukawa couplings do not play any role. 

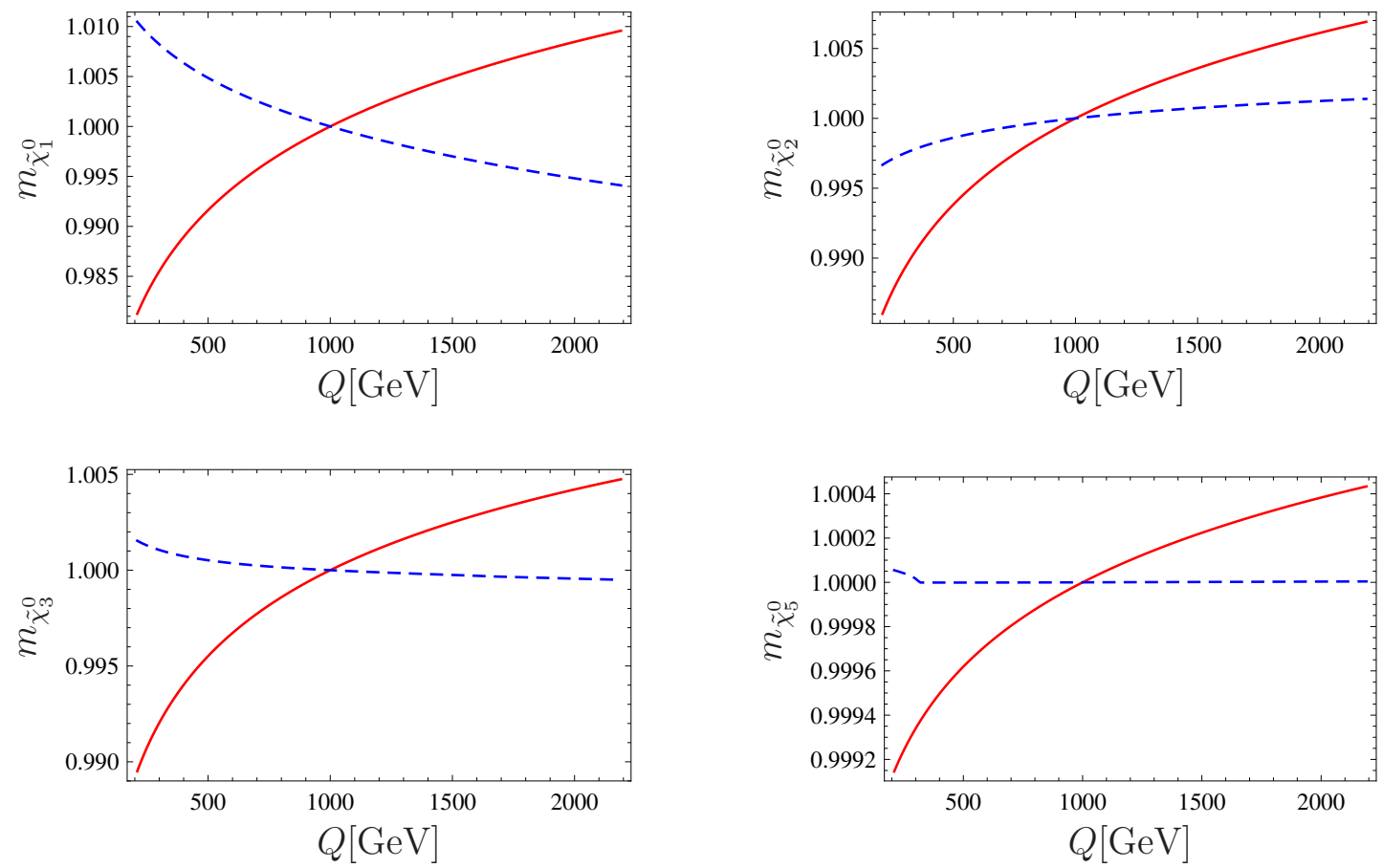

Figure 3. Dependence of the masses of the light neutralinos on the renormalization scale $Q$ at tree (red) and 1-loop level (dashed blue) normalized to the value at $Q=1 \mathrm{TeV}$ : From left to right and from above to below: $m_{\tilde{\chi}_{1}^{0}}, m_{\tilde{\chi}_{2}^{0}}, m_{\tilde{\chi}_{3}^{0}}$ and $m_{\tilde{\chi}_{5}^{0}}$.
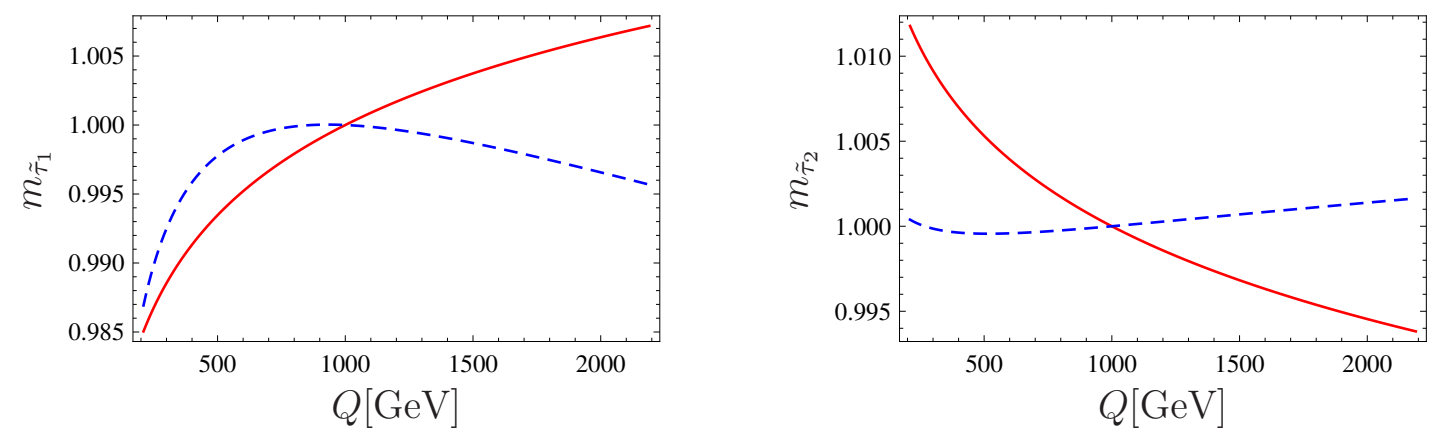

Figure 4. Dependence of the stau masses of the sleptons on the renormalization scale $Q$ at tree (red) and 1-loop level (dashed blue) normalized to the value at $Q=1 \mathrm{TeV}$.

\section{Comparison with the literature}

To date, the program package NMSSM-Tools [30-32] has been the only complete spectrum calculator for the NMSSM. NMSSM-Tools uses for the constrained NMSSM the parameters $m_{0}, M_{1 / 2}, A_{0}$ and $A_{\kappa}$ at the GUT scale whereas $\tan \beta$ and $\lambda$ are given at the electroweak scale. Moreover, in NMSSM-Tools the tadpole equations are solved with respect to $\left|v_{s}\right|, \kappa$, and $m_{S}^{2}$. We have performed a detailed numerical comparison of our implementation with the version 2.3 .1 and present here a few typical examples. 


\subsection{Differences between the programs}

Since both programs use different methods to calculate the spectrum, we have also done a comparison where we modified the codes such that both codes use equivalent methods except for small details. First, the implementation of NMSSM-Tools involves two different scales, namely the SUSY scale defined as

$$
Q_{\mathrm{SUSY}}^{2}=M_{\mathrm{SUSY}}^{2}=\frac{1}{4}\left(2 m_{\tilde{q}_{11}}^{2}+m_{\tilde{u}_{11}}^{2}+m_{\tilde{d}_{11}}^{2}\right)
$$

and the scale at which the masses are calculated,

$$
Q_{\mathrm{STSB}}^{2}=m_{\tilde{q}_{3}} m_{\tilde{u}_{3}} .
$$

In SPheno, all masses are evaluated at the SUSY scale, so that we had to set $Q_{\mathrm{STSB}}=$ $Q_{\mathrm{SUSY}}$ in the relevant routines of NMSSM-Tools. Second, as already stated in section 3.2, the two-loop $\beta$ function of $A_{\lambda}$ has been corrected in the public version of NMSSM-Tools. However, in general the numerical effect on the spectrum is rather small.

In the Higgs sector the loop contributions are taken into account differently in both codes. While SPheno takes the complete one-loop correction including the dependence of the external momenta, NMSSM-Tools uses the effective potential approach, e.g. setting the external momenta to zero. NMSSM-Tools calculates afterwards the momentum dependent contributions from top and bottom quarks. Also the included contributions differ: in SPheno the complete one-loop corrections to both, scalar and pseudo scalar Higgs bosons, and the two-loop contributions as given in ref. [33] are included. In NMSSM-Tools beside the dominant contributions due to third generation sfermions also electroweak corrections and some leading two-loop corrections for the scalars are calculated, while for the pseudoscalars only the dominant one-loop corrections due to tops, stops, bottoms, and sbottoms are included. In addition, some corrections due to charginos and neutralinos are absorbed in a redefined $A_{\lambda}$. To account for these differences we have switched off the two-loop parts in both codes. Furthermore, we have set the external momenta of the loop-diagrams of scalars in SPheno to zero. Finally, we have kept only those corrections to the pseudo-scalar masses in SPheno which are also included in NMSSM-Tools, but neglected the additional corrections absorbed in $A_{\lambda}$. In the following, we refer to these modified versions by SPheno mod and NMSSM-Tools mod, respectively.

Also in the chargino and neutralino sector the implementations are different: in SPheno the complete one-loop corrections are implemented whereas in NMSSM-Tools the corrections to the parameters $M_{1}, M_{2}$, and $\mu_{\text {eff }}$ are taken into account. In the slepton sector the differences are largest: SPheno contains the complete one-loop corrections whereas in NMSSM-Tools the calculation is done at tree-level. Last but not least we note that the data transfer has been done using the SLHA2 conventions [45]. 


\subsection{Results of the comparison}

As a first reference scenario, we take the benchmark point 1 proposed in ref. [40]. The corresponding input parameters for NMSSM-Tools are

$$
\begin{aligned}
& m_{0}=180 \mathrm{GeV}, \quad m_{1 / 2}=500 \mathrm{GeV}, \quad A_{0}=-1500 \mathrm{GeV}, \quad \tan \beta=10, \\
& \lambda^{\mathrm{SUSY}}=0.1, \quad A_{\kappa}^{\mathrm{GUT}}=-33.45, \quad \mu_{\mathrm{eff}}>0 .
\end{aligned}
$$

In the following we will vary $m_{0}$ and the keep the other parameters to the values shown here.

In the left graph of figure 5, we show the mass of the lightest scalar $h_{1}$ as a function of $m_{0}$. The largest discrepancies arise for the lighter scalar and pseudo scalar boson, where the relative differences between the complete calculation of both programs amount up to 2.5 and $35 \%$, respectively. In case of $h_{1}^{0}$ this is a combination of the $p^{2}$ terms in the loop-functions and the additional two-loop contributions. The differences in case of $A_{1}^{0}$ can easily be understood by noting that in NMSSM-Tools only the contribution of thirdgeneration sfermions are taken into account whereas we include the complete one-loop corrections plus the known two-loop contributions. In case of the modified program codes these differences reduce to at most $2 \%$ which is meanly due to two differences: (i) the way the top Yukawa coupling is calculated and (ii) the way the tadpole equations are solved which leads to somewhat different values between the two programs. There is no visible difference between NMSSM-Tools and NMSSM-Tools mod for the pseudo scalar and the heavy scalars. The reason is that in the case of the pseudo scalar no two-loop corrections are calculated in NMSSM-Tools and in case of the heavy scalars they are very small.

Finally, we have also cross-checked our results in the Higgs sector with ref. [33] and we have found agreement better than one per-mill when using the set of soft SUSY parameters at the scale $Q_{\text {STSB }}$. This small difference is an effect of the Yukawa and scalar-trilinear couplings of the first two generations which we take also into account. If we restrict ourself to third generation couplings there is an exact agreement between both calculations.

Concerning the chargino and neutralino masses, the agreement between the two spectrum calculators is rather good as can be seen in figure 6 . The relative differences are at most $1 \%$ and in general slightly below $0.5 \%$. In case of the sleptons the differences are more pronounced as can be seen in figure 7 which is due to the differences between treelevel and one-loop calculation and amounts in up to $3 \%$ and $0.6 \%$ for the light and heavy stau, respectively. Note, that although for LHC physics one expects similar experimental uncertainties, this precision necessary for a future linear collider or dark matter calculation require the inclusion of the radiative corrections to the slepton masses.

\section{Effects on the relic density of dark matter}

It is well known that the prediction of the dark matter relic density $\Omega_{\mathrm{CDM}} h^{2}$ is very sensitive to the exact mass configuration of the scenario under consideration [46]. For a neutralino LSP, this is, e.g., the case for the annihilation through Higgs-resonances, but also in the case of neutralino-sfermion co-annihilation. For the latter, the mass difference between the 

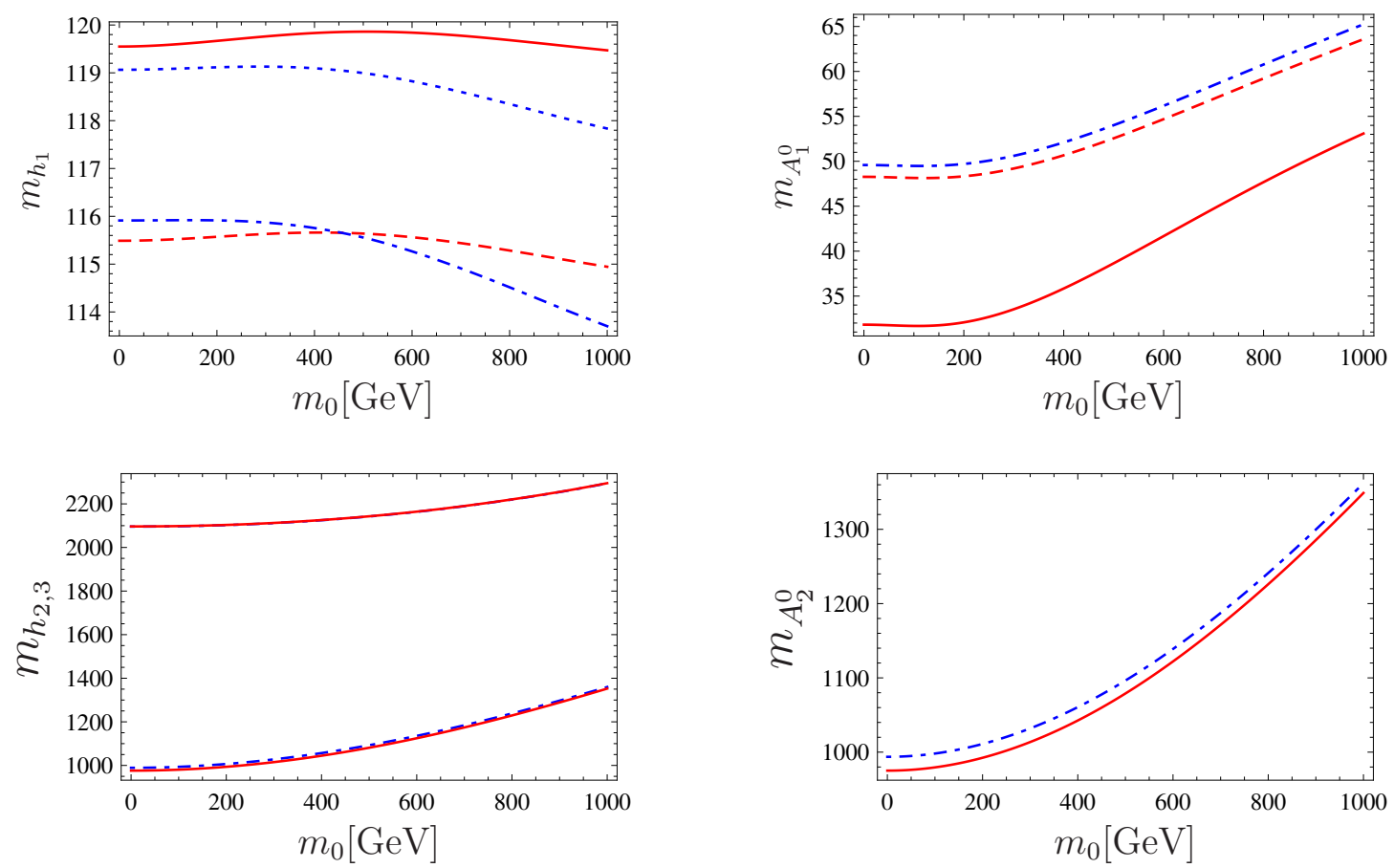

Figure 5. Comparison of the masses in GeV of the lightest scalar (upper left), the lightest pseudo scalar (upper right), heavier scalar masses (lower left) and heavier pseudo scalar mass (lower right) as a function of $m_{0}$ (in GeV). All other parameters are fixed as in eq. (4.3). The lines correspond to: are for unmodified version of SPheno (full red), NMSSM-Tools (dotted blue), SPheno mod (dashed red) and NMSSM-Tools mod (dot-dashed blue).

two particles plays a key role in the calculation of the resulting relic density. Therefore, it is necessary to calculate the complete spectrum as precisely as possible to get viable results of allowed regions of parameter space with respect to the constraints imposed by the presence of dark matter. Let us recall that recent measurements by the WMAP satellite in combination with further cosmological data lead to the favored interval

$$
0.1018<\Omega_{\mathrm{CDM}} h^{2}<0.1228
$$

at $3 \sigma$ confidence level [47].

We compute the relic density of the lightest neutralino using the public program package micrOMEGAs 2.4.0 [48]. To this end, we have implemented the NMSSM particle content and corresponding interactions into a model file for CalcHEP [49], which is used by micrOMEGAs to evaluate the (co)annihilation cross-section. The relevant interactions have again been calculated and written into the model files by the program package SARAH. Let us note, that we take into account important QCD effects, such as the running strong coupling constant and the running quark masses [50-52]. 

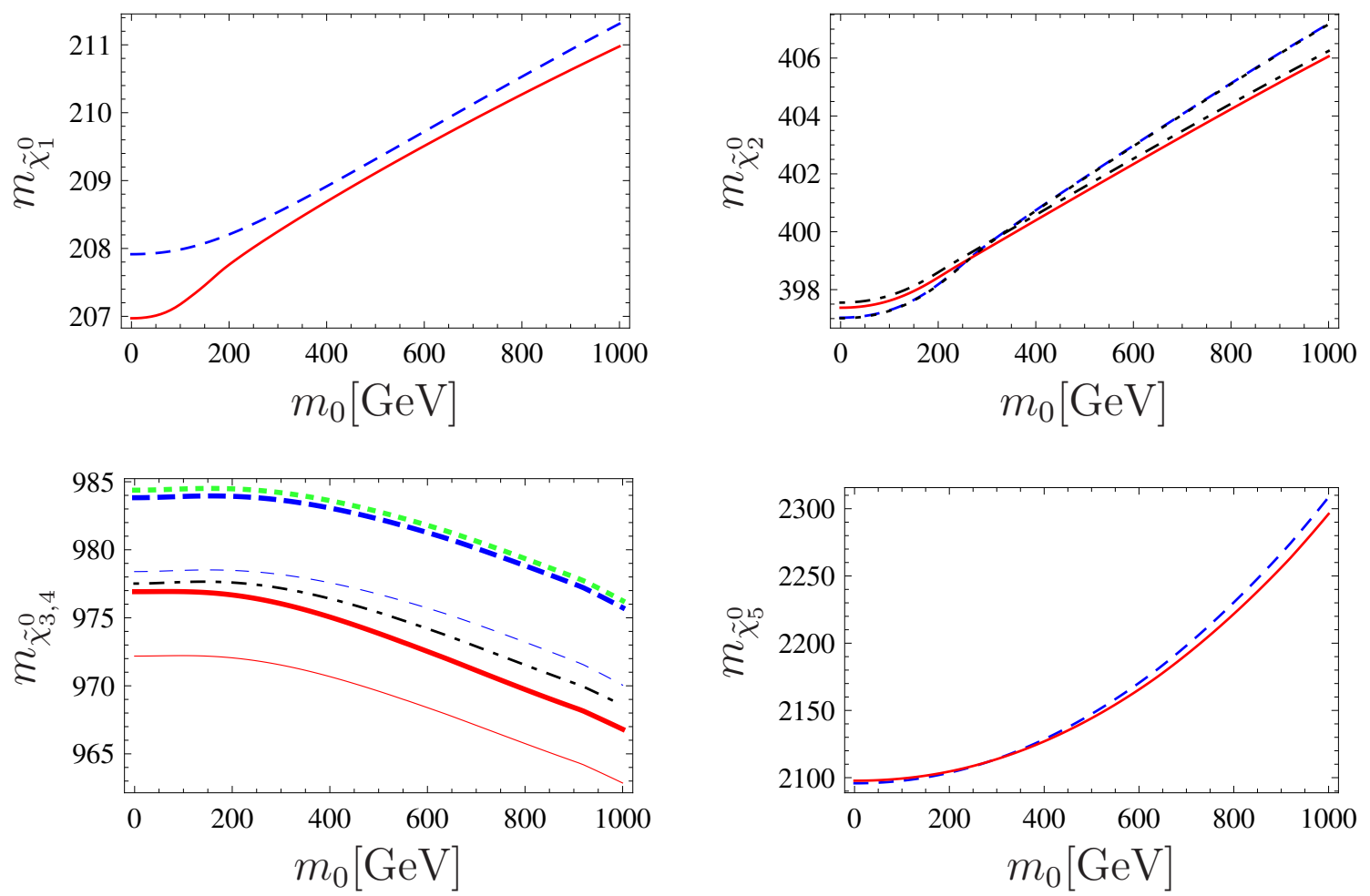

Figure 6. Comparison of chargino and neutralino masses (in $\mathrm{GeV}$ ) as a function of $m_{0}$ (in $\mathrm{GeV}$ ). All other parameters are fixed as in eq. (4.3). The lines correspond to the unmodified versions of SPheno (full red) and NMSSM-Tools (dashed blue). Up left: light neutralinos $\tilde{\chi}_{1}^{0}$. Up right: neutralino $\tilde{\chi}_{2}$ and chargino $\tilde{\chi}_{1}^{+}$(SPheno: black dotdashed, NMSSM-Tools: black dotted). Down left: neutralinos $\tilde{\chi}_{3}$ (thin lines), $\tilde{\chi}_{4}$ (thick lines) and chargino $\tilde{\chi}_{2}^{+}$(SPheno: black dotdashed, NMSSM-Tools: green dotted). Down right: $\tilde{\chi}_{5}$.
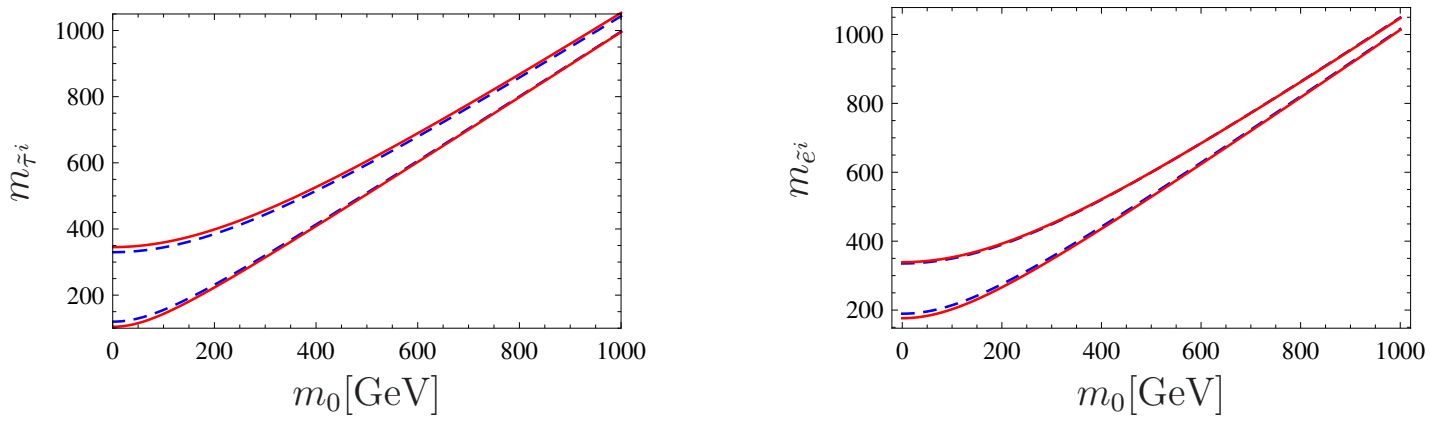

Figure 7. Comparison of selectron and stau masses (in $\mathrm{GeV}$ ) as a function of $m_{0}$. All other parameters are fixed as in eq. (4.3). The lines correspond to the unmodified versions of SPheno (full red) and NMSSM-Tools (dashed blue). 

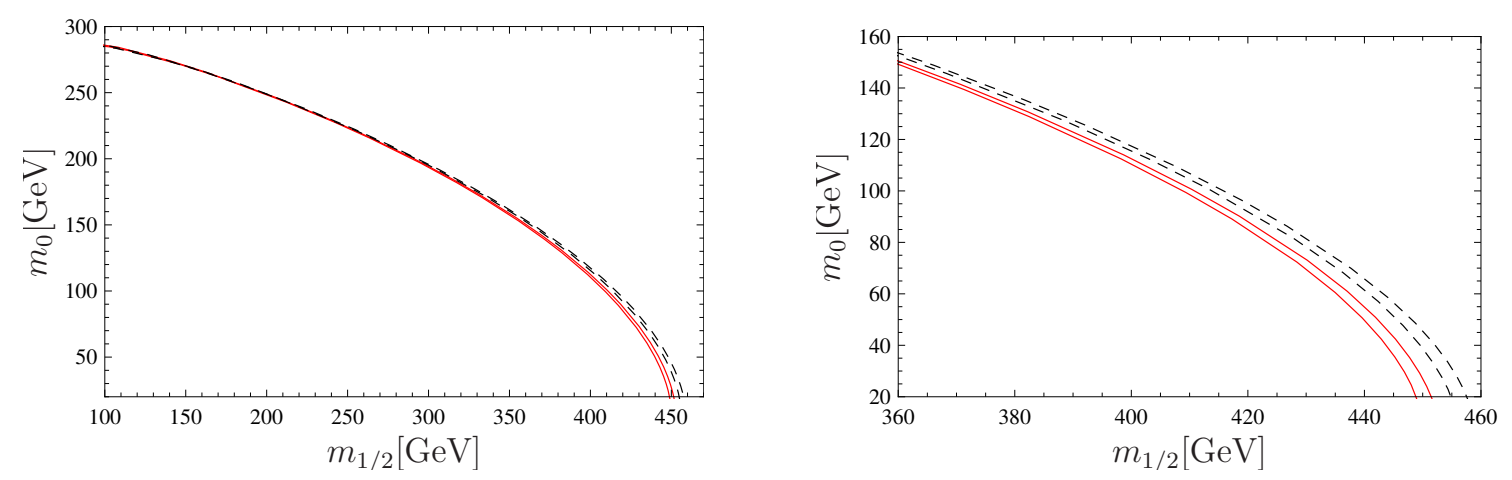

Figure 8. The isolines corresponding to $\Omega_{\mathrm{CDM}} h^{2}=0.1018$ and $\Omega_{\mathrm{CDM}} h^{2}=0.1228$ in the $m_{0}-m_{1 / 2}$ plane for dominant neutralino-stau coannihilations. All other parameters are fixed as in eq. (5.2). The red solid lines have been obtained for the complete mass spectrum at the one-loop level, while for the black dashed line the loop corrections to the slepton masses have been disabled. The right graph corresponds to a zoom into the left one.

As an example, we illustrate the effect of the one-loop correction to the slepton masses on the dark matter relic density in a region of dominant neutralino-stau coannihilations. In figure 8, we show the isolines corresponding to the upper and lower limit of eq. (5.1) in the $m_{0}-m_{1 / 2}$ plane. All remaining parameters of eq. (3.4) are fixed to

$$
\begin{gathered}
\tan \beta=15, \kappa^{\mathrm{SUSY}}=-0.05, \lambda^{\mathrm{SUSY}}=-0.1, \\
A_{\kappa}^{\mathrm{GUT}}=30 \mathrm{GeV}, A_{0}=A_{\lambda}^{\mathrm{GUT}}=1000 \mathrm{GeV}, v_{s}=2 \cdot 10^{4} \mathrm{GeV} .
\end{gathered}
$$

One clearly sees that the allowed parameter range gets shifted depending on the precision with which the spectrum is calculated. More, the two regions shown do not overlap as can also be clearly be seen in the figure to the right.

For a point with $\Omega_{\mathrm{CDM}} h^{2}=0.112$ at $m_{1 / 2} \simeq 451.2 \mathrm{GeV}$, the resulting one-loop corrected masses of the lightest neutralino and the lighter stau are $m_{\tilde{\chi}_{1}^{0}}=186.0 \mathrm{GeV}$ and $m_{\tilde{\tau}_{1}}=196.8 \mathrm{GeV}$, respectively. In consequence, coannihilations account for about $60 \%$ of the total annihilation cross-section, where the most important final states are $\tau h_{1}(27 \%)$ and $\tau Z^{0}(15 \%)$. A sizable contribution of about $14 \%$ (5\%) comes also from stau-antistau (stau-stau) annihilation. The remaining contributions are mainly from neutralino pair annihilation. For lower values of $m_{1 / 2} \lesssim 200 \mathrm{GeV}$, the coannihilations become less important within the WMAP-favored region, the dominant mechanism is then neutralino pair annihilation into $\tau^{+} \tau^{-}$pairs through stau-exchange.

\section{Conclusion}

The NMSSM is an attractive extension of the MSSM, in particular as it solves the $\mu$ problem of the MSSM and as it leads to new phenomenology at present and future collider experiments. It can also explain the observed amount of dark matter in the universe. However, in particular for comparison of the WMAP data improved theoretical predictions are necessary. We therefore have presented in this paper the complete one-loop calculation 
of the electroweak sector: Higgs bosons, charginos, neutralinos and sleptons. While in case of the Higgs bosons we have reproduced known results, the corrections to the other particles have not yet been discussed in the literature. We have shown that the corrections amount to the order of a few per-cent. While the corrections are most likely below the precision of the coming LHC data, they are clearly important for comparison with WMAP data and also with a future international linear collider, and thus crucial for precision investigations of the NMSSM parameter space.

\section{Acknowledgments}

We thank U. Ellwanger, C. Hugonie and P. Slavich for useful discussions. We thank P. Slavich in particular also for providing his fortran code of the one-loop and two-loop corrections to the Higgs masses. This work has been supported by the DFG, project number PO-1337/1-1. B.H. acknowledges support from the Hamburg Excellence Cluster "Connecting particles to the cosmos" and W.P. from the Alexander von Humboldt Foundation and the Spanish grant FPA2008-00319/FPA. F.S. has been supported by the DFG research training group GRK1147.

\section{A Squark mass matrices}

For completeness, we display here the mass matrices of the squarks, since they also enter the one-loop corrections and are particularly important in the case of the Higgs-bosons. In the basis $\left(\tilde{d}_{L}, \tilde{s}_{L}, \tilde{b}_{L}, \tilde{d}_{R}, \tilde{s}_{R}, \tilde{b}_{R}\right)$, the mass matrix for the down-type squarks is given by

$$
m_{T}^{2, \tilde{d}}=\left(\begin{array}{cc}
m_{L L}^{2, d} & \frac{1}{2}\left(\sqrt{2} v_{d} T_{d}^{T}-v_{s} v_{u} \lambda^{*} Y_{d}^{T}\right) \\
\frac{1}{2}\left(\sqrt{2} v_{d} T_{d}^{*}-v_{s} v_{u} \lambda Y_{d}^{*}\right) & m_{R R}^{2, d}
\end{array}\right),
$$

where the diagonal entries read

$$
\begin{aligned}
& m_{L L}^{2, d}=m_{\tilde{Q}}^{2}+\frac{v_{d}^{2}}{2} Y_{d}^{*} Y_{d}^{T}-\frac{3 g_{2}^{2}+g_{1}^{2}}{24}\left(v_{d}^{2}-v_{u}^{2}\right) \mathbf{1}_{3}, \\
& m_{R R}^{2, d}=m_{\tilde{D}}^{2}+\frac{v_{d}^{2}}{2} Y_{d}^{T} Y_{d}^{*}+\frac{g_{1}^{2}}{12}\left(v_{u}^{2}-v_{d}^{2}\right) \mathbf{1}_{3} .
\end{aligned}
$$

The corresponding expressions for the up-type squarks in the basis $\left(\tilde{u}_{L}, \tilde{c}_{L}, \tilde{t}_{L}, \tilde{u}_{R}, \tilde{c}_{R}, \tilde{t}_{R}\right)$ are

$$
m_{T}^{2, \tilde{u}}=\left(\begin{array}{cc}
m_{L L}^{2, u} & \frac{1}{2}\left(\sqrt{2} v_{u} T_{u}^{T}-v_{d} v_{s} \lambda^{*} Y_{u}^{T}\right) \\
\frac{1}{2}\left(\sqrt{2} v_{u} T_{u}^{*}-v_{d} v_{s} \lambda Y_{u}^{*}\right) & m_{R R}^{2, u}
\end{array}\right)
$$

with

$$
\begin{aligned}
& m_{L L}^{2, u}=m_{\tilde{Q}}^{2}+\frac{v_{u}^{2}}{2} Y_{u}^{*} Y_{u}^{T}+\frac{3 g_{2}^{2}-g_{1}^{2}}{24}\left(v_{d}^{2}-v_{u}^{2}\right) \mathbf{1}_{3}, \\
& m_{R R}^{2, u}=m_{\tilde{U}}^{2}+\frac{v_{u}^{2}}{2} Y_{u}^{T} Y_{u}^{*}+\frac{g_{1}^{2}}{6}\left(v_{d}^{2}-v_{u}^{2}\right) \mathbf{1}_{3}
\end{aligned}
$$

These matrix are diagonalized by unitary mixing matrices $Z^{Q}$ :

$$
Z^{Q} m_{T}^{2, \tilde{q}} Z^{Q \dagger}=m_{\operatorname{diag}}^{2, \tilde{q}}, q=d, u
$$




\section{B Anomalous dimensions}

In this appendix, we give the detailed expressions of the anomalous dimension of the Higgsfields, which are needed for the RGE evaluation of the VEVs.

$$
\begin{aligned}
\gamma_{\hat{H}_{d}}^{(1)}= & 3 \operatorname{Tr}\left(Y_{d} Y_{d}^{\dagger}\right)-\frac{3}{10} g_{1}^{2}-\frac{3}{2} g_{2}^{2}+|\lambda|^{2}+\operatorname{Tr}\left(Y_{e} Y_{e}^{\dagger}\right) \\
\gamma_{\hat{H}_{d}}^{(2)}= & \frac{207}{100} g_{1}^{4}+\frac{9}{10} g_{1}^{2} g_{2}^{2}+\frac{15}{4} g_{2}^{4}-2|\lambda|^{2}|\kappa|^{2}-3|\lambda|^{4}-\frac{2}{5} g_{1}^{2} \operatorname{Tr}\left(Y_{d} Y_{d}^{\dagger}\right) \\
& +16 g_{3}^{2} \operatorname{Tr}\left(Y_{d} Y_{d}^{\dagger}\right)+\frac{6}{5} g_{1}^{2} \operatorname{Tr}\left(Y_{e} Y_{e}^{\dagger}\right)-3|\lambda|^{2} \operatorname{Tr}\left(Y_{u} Y_{u}^{\dagger}\right)-9 \operatorname{Tr}\left(Y_{d} Y_{d}^{\dagger} Y_{d} Y_{d}^{\dagger}\right) \\
& -3 \operatorname{Tr}\left(Y_{d} Y_{d}^{\dagger} Y_{u} Y_{u}^{\dagger}\right)-3 \operatorname{Tr}\left(Y_{e} Y_{e}^{\dagger} Y_{e} Y_{e}^{\dagger}\right) \\
\gamma_{\hat{H}_{u}}^{(1)}= & 3 \operatorname{Tr}\left(Y_{u} Y_{u}^{\dagger}\right)-\frac{3}{10} g_{1}^{2}-\frac{3}{2} g_{2}^{2}+|\lambda|^{2} \\
\gamma_{\hat{H}_{u}}^{(2)}= & \frac{207}{100} g_{1}^{4}+\frac{9}{10} g_{1}^{2} g_{2}^{2}+\frac{15}{4} g_{2}^{4}-2|\lambda|^{2}|\kappa|^{2}-3|\lambda|^{4}-3|\lambda|^{2} \operatorname{Tr}\left(Y_{d} Y_{d}^{\dagger}\right) \\
& -|\lambda|^{2} \operatorname{Tr}\left(Y_{e} Y_{e}^{\dagger}\right)+\frac{4}{5} g_{1}^{2} \operatorname{Tr}\left(Y_{u} Y_{u}^{\dagger}\right)+16 g_{3}^{2} \operatorname{Tr}\left(Y_{u} Y_{u}^{\dagger}\right)-3 \operatorname{Tr}\left(Y_{d} Y_{d}^{\dagger} Y_{u} Y_{u}^{\dagger}\right) \\
& -9 \operatorname{Tr}\left(Y_{u} Y_{u}^{\dagger} Y_{u} Y_{u}^{\dagger}\right) \\
\gamma_{\hat{s}}^{(1)}= & 2|\kappa|^{2}+2|\lambda|^{2} \\
\gamma_{\hat{s}}^{(2)}= & \frac{6}{5} g_{1}^{2}|\lambda|^{2}+6 g_{2}^{2}|\lambda|^{2}-8|\kappa|^{4}-8|\lambda|^{2}|\kappa|^{2}-4|\lambda|^{4}-6|\lambda|^{2} \operatorname{Tr}\left(Y_{d} Y_{d}^{\dagger}\right) \\
& -2|\lambda|^{2} \operatorname{Tr}\left(Y_{e} Y_{e}^{\dagger}\right)-6|\lambda|^{2} \operatorname{Tr}\left(Y_{u} Y_{u}^{\dagger}\right)
\end{aligned}
$$

\section{Couplings}

We list in the following all couplings of the NMSSM which contribute to the electroweak self-energies or influence the annihilation or coannihilation of the neutralino. These and all other couplings of the NMSSM can be derived with the command MakeVertexList [EWSB] of SARAH. A pdf version is also available at [43].

We define the following abbreviations:

$$
\begin{aligned}
\tilde{\lambda} & \equiv+g_{1}^{2}+g_{2}^{2}-4 \lambda^{2} \\
\bar{\lambda} & \equiv g_{2}^{2}-2 \lambda^{2} \\
g_{-}^{2} & \equiv g_{2}^{2}-g_{1}^{2} \\
g_{+}^{2} & \equiv g_{1}^{2}+g_{2}^{2} \\
\Lambda_{1} & \equiv 2 v_{s} \kappa \lambda^{*}+2 v_{s} \lambda \kappa^{*}+\sqrt{2} 2 \operatorname{Re}\left\{T_{\lambda}\right\} \\
\Lambda_{2} & \equiv-2 v_{u} \lambda+v_{d} \kappa \\
\Lambda_{3} & \equiv-2 v_{d} \lambda+v_{u} \kappa
\end{aligned}
$$

Furthermore, $c_{\Theta}$ is $\cos \left(\Theta_{W}\right)$ and $s_{\Theta}$ is $\sin \left(\Theta_{W}\right)$. 


\section{C.1 Two fermion - one scalar}

$$
\begin{aligned}
& \Gamma_{\tilde{\chi}_{t_{1}}^{0} \tilde{\chi}_{t_{2}}^{0} h_{t_{3}}}^{L}=\frac{i}{2}\left(-g_{2} N_{t_{1} 2}^{*} N_{t_{2} 3}^{*} Z_{t_{3} 1}^{H}+\sqrt{2} \lambda N_{t_{1} 5}^{*} N_{t_{2} 4}^{*} Z_{t_{3} 1}^{H}+\sqrt{2} \lambda N_{t_{1} 4}^{*} N_{t_{2} 5}^{*} Z_{t_{3} 1}^{H}\right. \\
& -g_{1} N_{t_{1} 4}^{*} N_{t_{2} 1}^{*} Z_{t_{3} 2}^{H}+g_{2} N_{t_{1} 4}^{*} N_{t_{2} 2}^{*} Z_{t_{3} 2}^{H}+\sqrt{2} \lambda N_{t_{1} 5}^{*} N_{t_{2} 3}^{*} Z_{t_{3} 2}^{H}+g_{2} N_{t_{1} 2}^{*} N_{t_{2} 4}^{*} Z_{t_{3} 2}^{H} \\
& +g_{1} N_{t_{1} 1}^{*}\left(N_{t_{2} 3}^{*} Z_{t_{3} 1}^{H}-N_{t_{2} 4}^{*} Z_{t_{3} 2}^{H}\right)+\sqrt{2} \lambda N_{t_{1} 4}^{*} N_{t_{2} 3}^{*} Z_{t_{3} 3}^{H}-2 \sqrt{2} \kappa N_{t_{1} 5}^{*} N_{t_{2} 5}^{*} Z_{t_{3} 3}^{H} \\
& \left.+N_{t_{1} 3}^{*}\left(g_{1} N_{t_{2} 1}^{*} Z_{t_{3} 1}^{H}-g_{2} N_{t_{2} 2}^{*} Z_{t_{3} 1}^{H}+\sqrt{2} \lambda\left(N_{t_{2} 4}^{*} Z_{t_{3} 3}^{H}+N_{t_{2} 5}^{*} Z_{t_{3} 2}^{H}\right)\right)\right) \\
& \Gamma_{\tilde{\chi}_{t_{1}}^{0} \tilde{\chi}_{t_{2}}^{0} h_{t_{3}}}^{R}=\frac{i}{2}\left(Z _ { t _ { 3 } 1 } ^ { H } \left(N_{t_{1} 3}\left(g_{1} N_{t_{2} 1}-g_{2} N_{t_{2} 2}\right)+g_{1} N_{t_{1} 1} N_{t_{2} 3}-g_{2} N_{t_{1} 2} N_{t_{2} 3}+\sqrt{2} \lambda^{*} N_{t_{1} 5} N_{t_{2} 4}\right.\right. \\
& \left.+\sqrt{2} \lambda^{*} N_{t_{1} 4} N_{t_{2} 5}\right)+\sqrt{2} Z_{t_{3} 3}^{H}\left(-2 \kappa^{*} N_{t_{1} 5} N_{t_{2} 5}+\lambda^{*}\left(N_{t_{1} 3} N_{t_{2} 4}+N_{t_{1} 4} N_{t_{2} 3}\right)\right) \\
& +Z_{t_{3} 2}^{H}\left(N_{t_{1} 4}\left(-g_{1} N_{t_{2} 1}+g_{2} N_{t_{2} 2}\right)+\left(-g_{1} N_{t_{1} 1}+g_{2} N_{t_{1} 2}\right) N_{t_{2} 4}\right. \\
& \left.\left.+\sqrt{2} \lambda^{*}\left(N_{t_{1} 3} N_{t_{2} 5}+N_{t_{1} 5} N_{t_{2} 3}\right)\right)\right)
\end{aligned}
$$

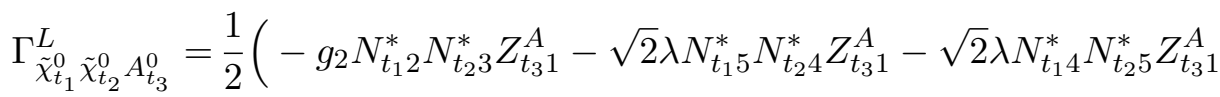

$$
\begin{aligned}
& -g_{1} N_{t_{1} 4}^{*} N_{t_{2} 1}^{*} Z_{t_{3} 2}^{A}+g_{2} N_{t_{1} 4}^{*} N_{t_{2} 2}^{*} Z_{t_{3} 2}^{A}-\sqrt{2} \lambda N_{t_{1} 5}^{*} N_{t_{2} 3}^{*} Z_{t_{3} 2}^{A}+g_{2} N_{t_{1} 2}^{*} N_{t_{2} 4}^{*} Z_{t_{3} 2}^{A} \\
& -N_{t_{1} 1}^{*}\left(-g_{1} N_{t_{2} 3}^{*} Z_{t_{3} 1}^{A}+g_{1} N_{t_{2} 4}^{*} Z_{t_{3} 2}^{A}\right)-\sqrt{2} \lambda N_{t_{1} 4}^{*} N_{t_{2} 3}^{*} Z_{t_{3} 3}^{A} \\
& +2 \sqrt{2} \kappa N_{t_{1} 5}^{*} N_{t_{2} 5}^{*} Z_{t_{3} 3}^{A}-N_{t_{1} 3}^{*}\left(-g_{1} N_{t_{2} 1}^{*} Z_{t_{3} 1}^{A}+g_{2} N_{t_{2} 2}^{*} Z_{t_{3} 1}^{A}\right. \\
& \left.\left.+\sqrt{2} \lambda\left(N_{t_{2} 4}^{*} Z_{t_{3} 3}^{A}+N_{t_{2} 5}^{*} Z_{t_{3} 2}^{A}\right)\right)\right)
\end{aligned}
$$

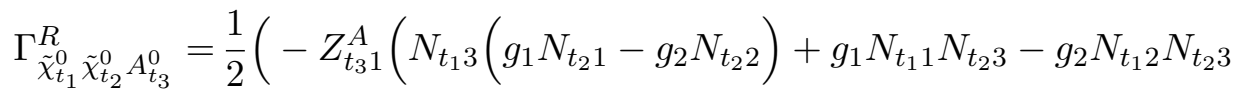

$$
\begin{aligned}
& \left.-\sqrt{2} \lambda^{*} N_{t_{1} 5} N_{t_{2} 4}-\sqrt{2} \lambda^{*} N_{t_{1} 4} N_{t_{2} 5}\right)+\sqrt{2} Z_{t_{3} 3}^{A}\left(-2 \kappa^{*} N_{t_{1} 5} N_{t_{2} 5}\right. \\
& \left.+\lambda^{*}\left(N_{t_{1} 3} N_{t_{2} 4}+N_{t_{1} 4} N_{t_{2} 3}\right)\right)+Z_{t_{3} 2}^{A}\left(N_{t_{1} 4}\left(g_{1} N_{t_{2} 1}-g_{2} N_{t_{2} 2}\right)\right. \\
& \left.\left.+\left(g_{1} N_{t_{1} 1}-g_{2} N_{t_{1} 2}\right) N_{t_{2} 4}+\sqrt{2} \lambda^{*}\left(N_{t_{1} 3} N_{t_{2} 5}+N_{t_{1} 5} N_{t_{2} 3}\right)\right)\right) \\
& \Gamma_{\tilde{\chi}_{t_{1}}^{0} \tilde{\chi}_{t_{2}}^{-} H_{t_{3}}^{+}}^{L}=i\left(-g_{2} V_{t_{2} 1}^{*} N_{t_{1} 3}^{*} Z_{t_{3} 1}^{+}+V_{t_{2} 2}^{*}\left(\frac{1}{\sqrt{2}} g_{1} N_{t_{1} 1}^{*} Z_{t_{3} 1}^{+}+\frac{1}{\sqrt{2}} g_{2} N_{t_{1} 2}^{*} Z_{t_{3} 1}^{+}-\lambda N_{t_{1} 5}^{*} Z_{t_{3} 2}^{+}\right)\right) \\
& \Gamma_{\chi_{t_{1}} \tilde{\chi}_{t_{2}}^{-} H_{t_{3}}^{+}}^{R}=i\left(-\frac{1}{2}\left(2 g_{2} U_{t_{2} 1} N_{t_{1} 4}+\sqrt{2} U_{t_{2} 2}\left(g_{1} N_{t_{1} 1}+g_{2} N_{t_{1} 2}\right)\right) Z_{t_{3} 2}^{+}-\lambda^{*} U_{t_{2} 2} N_{t_{1} 5} Z_{t_{3} 1}^{+}\right) \\
& \Gamma_{\tilde{\chi}_{t_{1}}^{-} \tilde{\chi}_{t_{2}}^{+} h_{t_{3}}}^{L}=-i \frac{1}{\sqrt{2}}\left(g_{2} V_{t_{2} 1}^{*} U_{t_{1} 2}^{*} Z_{t_{3} 2}^{H}+V_{t_{2} 2}^{*}\left(g_{2} U_{t_{1} 1}^{*} Z_{t_{3} 1}^{H}+\lambda U_{t_{1} 2}^{*} Z_{t_{3} 3}^{H}\right)\right) \\
& \Gamma_{\tilde{\chi}_{t_{1}}^{-} \tilde{\chi}_{t_{2}}^{+} h_{t_{3}}}^{R}=-i \frac{1}{\sqrt{2}}\left(g_{2} V_{t_{1} 1} U_{t_{2} 2} Z_{t_{3} 2}^{H}+V_{t_{1} 2}\left(g_{2} U_{t_{2} 1} Z_{t_{3} 1}^{H}+\lambda^{*} U_{t_{2} 2} Z_{t_{3} 3}^{H}\right)\right) \\
& \Gamma_{\tilde{\chi}_{t_{1}}^{-} \tilde{\chi}_{t_{2}}^{+} A_{t_{3}}^{0}}^{L}=-\frac{1}{\sqrt{2}}\left(g_{2} V_{t_{2} 1}^{*} U_{t_{1} 2}^{*} Z_{t_{3} 2}^{A}+V_{t_{2} 2}^{*}\left(g_{2} U_{t_{1} 1}^{*} Z_{t_{3} 1}^{A}-\lambda U_{t_{1} 2}^{*} Z_{t_{3} 3}^{A}\right)\right) \\
& \Gamma_{\tilde{\chi}_{t_{1}}^{-} \tilde{\chi}_{t_{2}}^{+} A_{t_{3}}^{0}}^{R}=\frac{1}{\sqrt{2}}\left(g_{2} V_{t_{1} 1} U_{t_{2} 2} Z_{t_{3} 2}^{A}+V_{t_{1} 2}\left(g_{2} U_{t_{2} 1} Z_{t_{3} 1}^{A}-\lambda^{*} U_{t_{2} 2} Z_{t_{3} 3}^{A}\right)\right)
\end{aligned}
$$




$$
\begin{aligned}
& \Gamma_{\bar{\nu}_{t_{1}} \tilde{\chi}_{t_{2}}^{0} \tilde{\nu}_{t_{3}}}^{R}=i \frac{1}{\sqrt{2}} Z_{t_{3} t_{1}}^{\nu, *}\left(g_{1} N_{t_{2} 1}-g_{2} N_{t_{2} 2}\right) \\
& \Gamma_{\bar{\nu}_{t_{1}} \tilde{\chi}_{t_{2}}^{+} \tilde{e}_{t_{3}}}^{R}=i\left(-g_{2} Z_{t_{3} t_{1}}^{E, *} V_{t_{2} 1}+\sum_{j_{1}=1}^{3} Y_{e, t_{1} j_{1}}^{*} Z_{t_{3} 3+j_{1}}^{E, *} V_{t_{2} 2}\right) \\
& \Gamma_{\bar{\nu}_{t_{1}} e_{t_{2}} H_{t_{3}}^{+}}^{R}=i \sum_{j_{1}=1}^{3} Y_{e, t_{1} j_{1}}^{*} U_{R, t_{2} j_{1}}^{e} Z_{t_{3} 1}^{+} \\
& \Gamma_{\tilde{\chi}_{t_{1}}^{0} e_{t_{2}} \tilde{e}_{t_{3}}^{*}}^{L}=i\left(\frac{1}{\sqrt{2}} g_{1} N_{t_{1} 1}^{*} \sum_{j_{1}=1}^{3} U_{L, t_{2} j_{1}}^{e, *} Z_{t_{3} j_{1}}^{E}+\frac{1}{\sqrt{2}} g_{2} N_{t_{1} 2}^{*} \sum_{j_{1}=1}^{3} U_{L, t_{2} j_{1}}^{e, *} Z_{t_{3} j_{1}}^{E}\right. \\
& \left.-N_{t_{1} 3}^{*} \sum_{j_{2}=1}^{3} \sum_{j_{1}=1}^{3} U_{L, t_{2} j_{1}}^{e, *} Y_{e, j_{1} j_{2}} Z_{t_{3} 3+j_{2}}^{E}\right) \\
& \Gamma_{\tilde{\chi}_{t_{1}}^{0} e_{t_{2}} \tilde{e}_{t_{3}}^{*}}^{R}=i\left(-\sqrt{2} g_{1} \sum_{j_{1}=1}^{3} Z_{t_{3} 3+j_{1}}^{E} U_{R, t_{2} j_{1}}^{e} N_{t_{1} 1}-\sum_{j_{2}=1}^{3} \sum_{j_{1}=1}^{3} Y_{e, j_{1} j_{2}}^{*} Z_{t_{3} j_{1}}^{E} U_{R, t_{2} j_{2}}^{e} N_{t_{1} 3}\right) \\
& \Gamma_{\tilde{\chi}_{t_{1}}^{0} d_{t_{2} \alpha_{2}} \tilde{t}_{t_{3} \alpha_{3}}^{*}}^{L}=-\frac{i}{6} \delta_{\alpha_{2}, \alpha_{3}}\left(\sqrt{2} g_{1} N_{t_{1} 1}^{*} \sum_{j_{1}=1}^{3} U_{L, t_{2} j_{1}}^{d, *} Z_{t_{3} j_{1}}^{D}-3 \sqrt{2} g_{2} N_{t_{1} 2}^{*} \sum_{j_{1}=1}^{3} U_{L, t_{2} j_{1}}^{d, * *} Z_{t_{3} j_{1}}^{D}\right. \\
& \left.+6 N_{t_{1} 3}^{*} \sum_{j_{2}=1}^{3} \sum_{j_{1}=1}^{3} U_{L, t_{2} j_{1}}^{d, *} Y_{d, j_{1} j_{2}} Z_{t_{3} 3+j_{2}}^{D}\right) \\
& \Gamma_{\tilde{\chi}_{t_{1}}^{0} d_{t_{2} \alpha_{2}} \tilde{d}_{t_{3} \alpha_{3}}^{*}}^{R}=-\frac{i}{3} \delta_{\alpha_{2}, \alpha_{3}}\left(\sqrt{2} g_{1} \sum_{j_{1}=1}^{3} Z_{t_{3} 3+j_{1}}^{D} U_{R, t_{2} j_{1}}^{d} N_{t_{1} 1}+3 \sum_{j_{2}=1}^{3} \sum_{j_{1}=1}^{3} Y_{d, j_{1} j_{2}}^{*} Z_{t_{3} j_{1}}^{D} U_{R, t_{2} j_{2}}^{d} N_{t_{1} 3}\right) \\
& \Gamma_{\tilde{\chi}_{t_{1}}^{0} u_{t_{2} \alpha_{2}} \tilde{u}_{t_{3} \alpha_{3}}^{*}}^{L}=-\frac{i}{6} \delta_{\alpha_{2}, \alpha_{3}}\left(\sqrt{2} g_{1} N_{t_{1} 1}^{*} \sum_{j_{1}=1}^{3} U_{L, t_{2} j_{1}}^{u, *} Z_{t_{3} j_{1}}^{U}+3 \sqrt{2} g_{2} N_{t_{1} 2}^{*} \sum_{j_{1}=1}^{3} U_{L, t_{2} j_{1}}^{u, *} Z_{t_{3} j_{1}}^{U}\right. \\
& \left.+6 N_{t_{1} 4}^{*} \sum_{j_{2}=1}^{3} \sum_{j_{1}=1}^{3} U_{L, t_{2} j_{1}}^{u, *} Y_{u, j_{1} j_{2}} Z_{t_{3} 3+j_{2}}^{U}\right) \\
& \Gamma_{\tilde{\chi}_{t_{1}}^{0} u_{t_{2} \alpha_{2}} \tilde{u}_{t_{3} \alpha_{3}}^{*}}^{R}=\frac{i}{3} \delta_{\alpha_{2}, \alpha_{3}}\left(2 \sqrt{2} g_{1} \sum_{j_{1}=1}^{3} Z_{t_{3} 3+j_{1}}^{U} U_{R, t_{2} j_{1}}^{u} N_{t_{1} 1}-3 \sum_{j_{2}=1}^{3} \sum_{j_{1}=1}^{3} Y_{u, j_{1} j_{2}}^{*} Z_{t_{3} j_{1}}^{U} U_{R, t_{2} j_{2}}^{u} N_{t_{1} 4}\right) \\
& \Gamma_{\tilde{\chi}_{t_{1}}^{+} e_{t_{2}} \tilde{\nu}_{t_{3}}^{*}}^{L}=-i g_{2} U_{t_{1} 1}^{*} \sum_{j_{1}=1}^{3} U_{L, t_{2} j_{1}}^{e, *} Z_{t_{3} j_{1}}^{\nu} \\
& \Gamma_{\tilde{\chi}_{t_{1}}^{+} e_{t_{2}} \tilde{\nu}_{t_{3}}^{*}}^{R}=i \sum_{j_{2}=1}^{3} \sum_{j_{1}=1}^{3} Y_{e, j_{1} j_{2}}^{*} Z_{t_{3} j_{1}}^{\nu} U_{R, t_{2} j_{2}}^{e} V_{t_{1} 2} \\
& \Gamma_{\tilde{\chi}_{t_{1}}^{+} d_{t_{2} \alpha_{2}} \tilde{u}_{t_{3} \alpha_{3}}^{*}}^{L}=i \delta_{\alpha_{2}, \alpha_{3}}\left(-g_{2} U_{t_{1} 1}^{*} \sum_{j_{1}=1}^{3} U_{L, t_{2} j_{1}}^{d, *} Z_{t_{3} j_{1}}^{U}+U_{t_{1} 2}^{*} \sum_{j_{2}=1}^{3} \sum_{j_{1}=1}^{3} U_{L, t_{2} j_{1}}^{d, *} Y_{u, j_{1} j_{2}} Z_{t_{3} 3+j_{2}}^{U}\right)
\end{aligned}
$$




$$
\begin{aligned}
& \Gamma_{\tilde{\chi}_{t_{1}}^{+} d_{t_{2} \alpha_{2}} \tilde{u}_{t_{3} \alpha_{3}}^{*}}^{R}=i \delta_{\alpha_{2}, \alpha_{3}} \sum_{j_{2}=1}^{3} \sum_{j_{1}=1}^{3} Y_{d, j_{1} j_{2}}^{*} Z_{t_{3} j_{1}}^{U} U_{R, t_{2} j_{2}}^{d} V_{t_{1} 2} \\
& \Gamma_{\bar{e}_{t_{1}} e_{t_{2}} h_{t_{3}}}^{L}=-i \frac{1}{\sqrt{2}} \sum_{j_{2}=1}^{3} U_{R, t_{1} j_{2}}^{e, *} \sum_{j_{1}=1}^{3} U_{L, t_{2} j_{1}}^{e, *} Y_{e, j_{1} j_{2}} Z_{t_{3} 1}^{H} \\
& \Gamma_{\bar{e}_{t_{1}} e_{t_{2}} h_{t_{3}}}^{R}=-i \frac{1}{\sqrt{2}} \sum_{j_{2}=1}^{3} \sum_{j_{1}=1}^{3} Y_{e, j_{1} j_{2}}^{*} U_{L, t_{1} j_{1}}^{e} U_{R, t_{2} j_{2}}^{e} Z_{t_{3} 1}^{H} \\
& \Gamma_{\bar{e}_{t_{1}} e_{t_{2}} A_{t_{3}}^{0}}^{L}=\frac{1}{\sqrt{2}} \sum_{j_{2}=1}^{3} U_{R, t_{1} j_{2}}^{e, *} \sum_{j_{1}=1}^{3} U_{L, t_{2} j_{1}}^{e, *} Y_{e, j_{1} j_{2}} Z_{t_{3} 1}^{A} \\
& \Gamma_{\bar{e}_{t_{1}} e_{t_{2}} A_{t_{3}}^{0}}^{R}=-\frac{1}{\sqrt{2}} \sum_{j_{2}=1}^{3} \sum_{j_{1}=1}^{3} Y_{e, j_{1} j_{2}}^{*} U_{L, t_{1} j_{1}}^{e} U_{R, t_{2} j_{2}}^{e} Z_{t_{3} 1}^{A} \\
& \Gamma_{\bar{d}_{t_{1} \alpha_{1}} d_{t_{2} \alpha_{2}} h_{t_{3}}}^{L}=-i \frac{1}{\sqrt{2}} \delta_{\alpha_{1}, \alpha_{2}} \sum_{j_{2}=1}^{3} U_{R, t_{1} j_{2}}^{d, *} \sum_{j_{1}=1}^{3} U_{L, t_{2} j_{1}}^{d, *} Y_{d, j_{1} j_{2}} Z_{t_{3} 1}^{H} \\
& \Gamma_{\bar{d}_{t_{1} \alpha_{1}} d_{t_{2} \alpha_{2}} h_{t_{3}}}^{R}=-i \frac{1}{\sqrt{2}} \delta_{\alpha_{1}, \alpha_{2}} \sum_{j_{2}=1}^{3} \sum_{j_{1}=1}^{3} Y_{d, j_{1} j_{2}}^{*} U_{L, t_{1} j_{1}}^{d} U_{R, t_{2} j_{2}}^{d} Z_{t_{3} 1}^{H} \\
& \Gamma_{\bar{d}_{t_{1} \alpha_{1}} d_{t_{2} \alpha_{2}} A_{t_{3}}^{0}}^{L}=\frac{1}{\sqrt{2}} \delta_{\alpha_{1}, \alpha_{2}} \sum_{j_{2}=1}^{3} U_{R, t_{1} j_{2}}^{d, *} \sum_{j_{1}=1}^{3} U_{L, t_{2} j_{1}}^{d, *} Y_{d, j_{1} j_{2}} Z_{t_{3} 1}^{A} \\
& \Gamma_{\bar{d}_{t_{1} \alpha_{1}} d_{t_{2} \alpha_{2}} A_{t_{3}}^{0}}^{R}=-\frac{1}{\sqrt{2}} \delta_{\alpha_{1}, \alpha_{2}} \sum_{j_{2}=1}^{3} \sum_{j_{1}=1}^{3} Y_{d, j_{1} j_{2}}^{*} U_{L, t_{1} j_{1}}^{d} U_{R, t_{2} j_{2}}^{d} Z_{t_{3} 1}^{A} \\
& \Gamma_{\bar{d}_{t_{1} \alpha_{1}} u_{t_{2} \alpha_{2}} H_{t_{3}}^{-}}^{L}=i Z_{t_{3} 1}^{+, *} \delta_{\alpha_{1}, \alpha_{2}} \sum_{j_{2}=1}^{3} U_{R, t_{1} j_{2}}^{d, *} \sum_{j_{1}=1}^{3} U_{L, t_{2} j_{1}}^{u, *} Y_{d, j_{1} j_{2}} \\
& \Gamma_{\bar{d}_{t_{1} \alpha_{1}} u_{t_{2} \alpha_{2}} H_{t_{3}}^{-}}^{R}=i Z_{t_{3} 2}^{+, *} \delta_{\alpha_{1}, \alpha_{2}} \sum_{j_{2}=1}^{3} \sum_{j_{1}=1}^{3} Y_{u, j_{1} j_{2}}^{*} U_{L, t_{1} j_{1}}^{d} U_{R, t_{2} j_{2}}^{u} \\
& \Gamma_{\bar{u}_{t_{1} \alpha_{1}} u_{t_{2} \alpha_{2}} h_{t_{3}}}^{L}=-i \frac{1}{\sqrt{2}} \delta_{\alpha_{1}, \alpha_{2}} \sum_{j_{2}=1}^{3} U_{R, t_{1} j_{2}}^{u, *} \sum_{j_{1}=1}^{3} U_{L, t_{2} j_{1}}^{u, *} Y_{u, j_{1} j_{2}} Z_{t_{3} 2}^{H} \\
& \Gamma_{\bar{u}_{t_{1} \alpha_{1}} u_{t_{2} \alpha_{2}} h_{t_{3}}}^{R}=-i \frac{1}{\sqrt{2}} \delta_{\alpha_{1}, \alpha_{2}} \sum_{j_{2}=1}^{3} \sum_{j_{1}=1}^{3} Y_{u, j_{1} j_{2}}^{*} U_{L, t_{1} j_{1}}^{u} U_{R, t_{2} j_{2}}^{u} Z_{t_{3} 2}^{H} \\
& \Gamma_{\bar{u}_{t_{1} \alpha_{1}} u_{t_{2} \alpha_{2}} A_{t_{3}}^{0}}^{L}=\frac{1}{\sqrt{2}} \delta_{\alpha_{1}, \alpha_{2}} \sum_{j_{2}=1}^{3} U_{R, t_{1} j_{2}}^{u, *} \sum_{j_{1}=1}^{3} U_{L, t_{2} j_{1}}^{u, *} Y_{u, j_{1} j_{2}} Z_{t_{3} 2}^{A} \\
& \Gamma_{\bar{u}_{t_{1} \alpha_{1}} u_{t_{2} \alpha_{2}} A_{t_{3}}^{0}}^{R}=-\frac{1}{\sqrt{2}} \delta_{\alpha_{1}, \alpha_{2}} \sum_{j_{2}=1}^{3} \sum_{j_{1}=1}^{3} Y_{u, j_{1} j_{2}}^{*} U_{L, t_{1} j_{1}}^{u} U_{R, t_{2} j_{2}}^{u} Z_{t_{3} 2}^{A}
\end{aligned}
$$

\section{C.2 Two fermion - one vector boson interaction}

$$
\Gamma_{\bar{\nu}_{t_{1}} \nu_{t_{2}} Z_{\mu}}^{L}=-\frac{i}{2} \delta_{t_{1}, t_{2}}\left(g_{1} s_{\Theta}+g_{2} c_{\Theta}\right)
$$




$$
\begin{aligned}
& \Gamma_{\bar{\nu}_{t_{1}} e_{t_{2}} W_{\mu}^{+}}^{L}=-i \frac{1}{\sqrt{2}} g_{2} U_{L, t_{2} t_{1}}^{e, *} \\
& \Gamma_{\tilde{\chi}_{t_{1}}^{0} \tilde{\chi}_{t_{2}}^{0} \gamma_{\mu}}^{L}=\frac{i}{2}\left(g_{1} c_{\Theta}-g_{2} s_{\Theta}\right)\left(N_{t_{2} 3}^{*} N_{t_{1} 3}-N_{t_{2} 4}^{*} N_{t_{1} 4}\right) \\
& \Gamma_{\tilde{\chi}_{t_{1}}^{0} \tilde{\chi}_{t_{2}}^{0} \gamma_{\mu}}^{R}=-\frac{i}{2}\left(g_{1} c_{\Theta}-g_{2} s_{\Theta}\right)\left(N_{t_{1} 3}^{*} N_{t_{2} 3}-N_{t_{1} 4}^{*} N_{t_{2} 4}\right) \\
& \Gamma_{\tilde{\chi}_{t_{1}}^{0} \tilde{\chi}_{t_{2}}^{0} Z_{\mu}}^{L}=-\frac{i}{2}\left(g_{1} s_{\Theta}+g_{2} c_{\Theta}\right)\left(N_{t_{2} 3}^{*} N_{t_{1} 3}-N_{t_{2} 4}^{*} N_{t_{1} 4}\right) \\
& \Gamma_{\tilde{\chi}_{t_{1}}^{0} \tilde{\chi}_{t_{2}}^{0} Z_{\mu}}^{R}=\frac{i}{2}\left(g_{1} s_{\Theta}+g_{2} c_{\Theta}\right)\left(N_{t_{1} 3}^{*} N_{t_{2} 3}-N_{t_{1} 4}^{*} N_{t_{2} 4}\right) \\
& \Gamma_{\tilde{\chi}_{t_{1}}^{0} \tilde{\chi}_{t_{2}}^{-} W_{\mu}^{+}}^{L}=-\frac{i}{2} g_{2}\left(2 V_{t_{2} 1}^{*} N_{t_{1} 2}+\sqrt{2} V_{t_{2} 2}^{*} N_{t_{1} 3}\right) \\
& \Gamma_{\tilde{\chi}_{t_{1}}^{0} \tilde{\chi}_{t_{2}}^{-} W_{\mu}^{+}}^{R}=-\frac{i}{2} g_{2}\left(2 N_{t_{1} 2}^{*} U_{t_{2} 1}-\sqrt{2} N_{t_{1} 4}^{*} U_{t_{2} 2}\right) \\
& \Gamma_{\tilde{\chi}_{t_{1}}^{+} \tilde{\chi}_{t_{2}}^{-} \gamma_{\mu}}^{L}=\frac{i}{2}\left(2 g_{2} V_{t_{2} 1}^{*} s_{\Theta} V_{t_{1} 1}+V_{t_{2} 2}^{*}\left(g_{1} c_{\Theta}+g_{2} s_{\Theta}\right) V_{t_{1} 2}\right) \\
& \Gamma_{\tilde{\chi}_{t_{1}}^{+} \tilde{\chi}_{t_{2}}^{-} \gamma_{\mu}}^{R}=\frac{i}{2}\left(2 g_{2} U_{t_{1} 1}^{*} s_{\Theta} U_{t_{2} 1}+U_{t_{1} 2}^{*}\left(g_{1} c_{\Theta}+g_{2} s_{\Theta}\right) U_{t_{2} 2}\right) \\
& \Gamma_{\tilde{\chi}_{t_{1}}^{+} \tilde{\chi}_{t_{2}}^{-} Z_{\mu}}^{L}=\frac{i}{2}\left(2 g_{2} V_{t_{2} 1}^{*} c_{\Theta} V_{t_{1} 1}+V_{t_{2} 2}^{*}\left(-g_{1} s_{\Theta}+g_{2} c_{\Theta}\right) V_{t_{1} 2}\right) \\
& \Gamma_{\tilde{\chi}_{t_{1}}^{+} \tilde{\chi}_{t_{2}}^{-} Z_{\mu}}^{R}=\frac{i}{2}\left(2 g_{2} U_{t_{1} 1}^{*} c_{\Theta} U_{t_{2} 1}+U_{t_{1} 2}^{*}\left(-g_{1} s_{\Theta}+g_{2} c_{\Theta}\right) U_{t_{2} 2}\right) \\
& \Gamma_{\bar{e}_{t_{1}} e_{t_{2}} \gamma_{\mu}}^{L}=\frac{i}{2} \delta_{t_{1}, t_{2}}\left(g_{1} c_{\Theta}+g_{2} s_{\Theta}\right) \\
& \Gamma_{\bar{e}_{t_{1}} e_{t_{2}} \gamma_{\mu}}^{R}=i g_{1} c_{\Theta} \delta_{t_{1}, t_{2}} \\
& \Gamma_{\bar{e}_{t_{1}} e_{t_{2}} Z_{\mu}}^{L}=\frac{i}{2} \delta_{t_{1}, t_{2}}\left(-g_{1} s_{\Theta}+g_{2} c_{\Theta}\right) \\
& \Gamma_{\bar{e}_{t_{1}} e_{t_{2}} Z_{\mu}}^{R}=-i g_{1} \delta_{t_{1}, t_{2}} s_{\Theta} \\
& \Gamma_{\bar{d}_{t_{1} \alpha_{1}}}^{L} d_{t_{2} \alpha_{2} \gamma_{\mu}}=-\frac{i}{6} \delta_{\alpha_{1}, \alpha_{2}} \delta_{t_{1}, t_{2}}\left(-3 g_{2} s_{\Theta}+g_{1} c_{\Theta}\right) \\
& \Gamma_{\bar{d}_{t_{1} \alpha_{1}} d_{t_{2} \alpha_{2} \gamma_{\mu}}^{R}}^{R}=\frac{i}{3} g_{1} c_{\Theta} \delta_{\alpha_{1}, \alpha_{2}} \delta_{t_{1}, t_{2}} \\
& \Gamma_{\bar{d}_{t_{1} \alpha_{1}} d_{t_{2} \alpha_{2}} Z_{\mu}}^{L}=\frac{i}{6} \delta_{\alpha_{1}, \alpha_{2}} \delta_{t_{1}, t_{2}}\left(3 g_{2} c_{\Theta}+g_{1} s_{\Theta}\right) \\
& \Gamma_{\bar{d}_{t_{1} \alpha_{1}}}^{R} d_{t_{2} \alpha_{2}} Z_{\mu}=-\frac{i}{3} g_{1} \delta_{\alpha_{1}, \alpha_{2}} \delta_{t_{1}, t_{2}} s_{\Theta} \\
& \Gamma_{\bar{d}_{t_{1} \alpha_{1}} u_{t_{2} \alpha_{2} W_{\mu}}^{L}}^{L}=-i \frac{1}{\sqrt{2}} g_{2} \delta_{\alpha_{1}, \alpha_{2}} \sum_{j_{1}=1}^{3} U_{L, t_{2} j_{1}}^{u, *} U_{L, t_{1} j_{1}}^{d} \\
& \Gamma_{\bar{u}_{t_{1} \alpha_{1}} u_{t_{2} \alpha_{2} \gamma_{\mu}}^{L}}^{L}=-\frac{i}{6} \delta_{\alpha_{1}, \alpha_{2}} \delta_{t_{1}, t_{2}}\left(3 g_{2} s_{\Theta}+g_{1} c_{\Theta}\right) \\
& \Gamma_{\bar{u}_{t_{1} \alpha_{1}} u_{t_{2} \alpha_{2} \gamma_{\mu}}^{R}}^{R}=-\frac{2 i}{3} g_{1} c_{\Theta} \delta_{\alpha_{1}, \alpha_{2}} \delta_{t_{1}, t_{2}} \\
& \Gamma_{\bar{u}_{t_{1} \alpha_{1}} u_{t_{2} \alpha_{2}} Z_{\mu}}^{L}=-\frac{i}{6} \delta_{\alpha_{1}, \alpha_{2}} \delta_{t_{1}, t_{2}}\left(3 g_{2} c_{\Theta}-g_{1} s_{\Theta}\right) \\
& \Gamma_{\bar{u}_{t_{1} \alpha_{1}} u_{t_{2} \alpha_{2}} Z_{\mu}}^{R}=\frac{2 i}{3} g_{1} \delta_{\alpha_{1}, \alpha_{2}} \delta_{t_{1}, t_{2}} s_{\Theta}
\end{aligned}
$$




\section{C.3 Two scalar - one vector boson interaction}

$$
\begin{aligned}
& \Gamma_{\tilde{d}_{t_{1} \alpha_{1}} \tilde{d}_{t_{2} \alpha_{2}}^{*} \gamma_{\mu}}=-\frac{i}{6} \delta_{\alpha_{1}, \alpha_{2}}\left(\left(-3 g_{2} s_{\Theta}+g_{1} c_{\Theta}\right) \sum_{j_{1}=1}^{3} Z_{t_{1} j_{1}}^{D, *} Z_{t_{2} j_{1}}^{D}-2 g_{1} c_{\Theta} \sum_{j_{1}=1}^{3} Z_{t_{1} 3+j_{1}}^{D, *} Z_{t_{2} 3+j_{1}}^{D}\right) \\
& \Gamma_{\tilde{d}_{t_{1} \alpha_{1}} \tilde{d}_{t_{2} \alpha_{2}}^{*} Z_{\mu}}=\frac{i}{6} \delta_{\alpha_{1}, \alpha_{2}}\left(\left(3 g_{2} c_{\Theta}+g_{1} s_{\Theta}\right) \sum_{j_{1}=1}^{3} Z_{t_{1} j_{1}}^{D, *} Z_{t_{2} j_{1}}^{D}-2 g_{1} s_{\Theta} \sum_{j_{1}=1}^{3} Z_{t_{1} 3+j_{1}}^{D, *} Z_{t_{2} 3+j_{1}}^{D}\right) \\
& \Gamma_{\tilde{\nu}_{t_{1}} \tilde{e}_{t_{2}}^{*} W_{\mu}^{-}}=-i \frac{1}{\sqrt{2}} g_{2} \sum_{j_{1}=1}^{3} Z_{t_{1} j_{1}}^{\nu, *} Z_{t_{2} j_{1}}^{E} \\
& \Gamma_{\tilde{\nu}_{t_{1}} \tilde{\nu}_{t_{2}}^{*} Z_{\mu}}=-\frac{i}{2} \sum_{j_{1}=1}^{3} Z_{t_{1} j_{1}}^{\nu, *} Z_{t_{2} j_{1}}^{\nu}\left(g_{1} s_{\Theta}+g_{2} c_{\Theta}\right) \\
& \Gamma_{\tilde{u}_{t_{1} \alpha_{1}} \tilde{d}_{t_{2} \alpha_{2}}^{*} W_{\mu}^{-}}=-i \frac{1}{\sqrt{2}} g_{2} \delta_{\alpha_{1}, \alpha_{2}} \sum_{j_{1}=1}^{3} Z_{t_{1} j_{1}}^{U, *} Z_{t_{2} j_{1}}^{D} \\
& \Gamma_{\tilde{u}_{t_{1} \alpha_{1}} \tilde{u}_{t_{2} \alpha_{2}}^{*} \gamma_{\mu}}=-\frac{i}{6} \delta_{\alpha_{1}, \alpha_{2}}\left(\left(3 g_{2} s_{\Theta}+g_{1} c_{\Theta}\right) \sum_{j_{1}=1}^{3} Z_{t_{1} j_{1}}^{U, *} Z_{t_{2} j_{1}}^{U}+4 g_{1} c_{\Theta} \sum_{j_{1}=1}^{3} Z_{t_{1} 3+j_{1}}^{U, *} Z_{t_{2} 3+j_{1}}^{U}\right) \\
& \Gamma_{\tilde{u}_{t_{1} \alpha_{1}} \tilde{u}_{t_{2} \alpha_{2}}^{*} Z_{\mu}}=-\frac{i}{6} \delta_{\alpha_{1}, \alpha_{2}}\left(\left(3 g_{2} c_{\Theta}-g_{1} s_{\Theta}\right) \sum_{j_{1}=1}^{3} Z_{t_{1} j_{1}}^{U, *} Z_{t_{2} j_{1}}^{U}-4 g_{1} s_{\Theta} \sum_{j_{1}=1}^{3} Z_{t_{1} 3+j_{1}}^{U, *} Z_{t_{2} 3+j_{1}}^{U}\right) \\
& \Gamma_{\tilde{e}_{t_{1}} \tilde{e}_{t_{2}}^{*} \gamma_{\mu}}=\frac{i}{2}\left(\left(g_{1} c_{\Theta}+g_{2} s_{\Theta}\right) \sum_{j_{1}=1}^{3} Z_{t_{1} j_{1}}^{E, *} Z_{t_{2} j_{1}}^{E}+2 g_{1} c_{\Theta} \sum_{j_{1}=1}^{3} Z_{t_{1} 3+j_{1}}^{E, *} Z_{t_{2} 3+j_{1}}^{E}\right) \\
& \Gamma_{\tilde{e}_{t_{1}} \tilde{e}_{t_{2}}^{*} Z_{\mu}}=\frac{i}{2}\left(\left(-g_{1} s_{\Theta}+g_{2} c_{\Theta}\right) \sum_{j_{1}=1}^{3} Z_{t_{1} j_{1}}^{E, *} Z_{t_{2} j_{1}}^{E}-2 g_{1} s_{\Theta} \sum_{j_{1}=1}^{3} Z_{t_{1} 3+j_{1}}^{E, *} Z_{t_{2} 3+j_{1}}^{E}\right) \\
& \Gamma_{h_{t_{1}} H_{t_{2}}^{+} W_{\mu}^{-}}=-\frac{i}{2} g_{2}\left(Z_{t_{1} 1}^{H} Z_{t_{2} 1}^{+}-Z_{t_{1} 2}^{H} Z_{t_{2} 2}^{+}\right) \\
& \Gamma_{h_{1} A_{t_{2}}^{0} Z_{\mu}}=\frac{1}{2}\left(-g_{1} s_{\Theta}-g_{2} c_{\Theta}\right)\left(Z_{t_{2} 1}^{A} Z_{t_{1} 1}^{H}-Z_{t_{2} 2}^{A} Z_{t_{1} 2}^{H}\right) \\
& \Gamma_{A_{t_{1}}^{0} H_{t_{2}}^{+} W_{\mu}^{-}}=\frac{1}{2} g_{2}\left(Z_{t_{1} 1}^{A} Z_{t_{2} 1}^{+}+Z_{t_{1} 2}^{A} Z_{t_{2} 2}^{+}\right) \\
& \Gamma_{H_{t_{1}}^{-} H_{t_{2}}^{+} \gamma_{\mu}}=\frac{i}{2}\left(g_{1} c_{\Theta}+g_{2} s_{\Theta}\right)\left(Z_{t_{1} 1}^{+, *} Z_{t_{2} 1}^{+}+Z_{t_{1} 2}^{+, *} Z_{t_{2} 2}^{+}\right) \\
& \Gamma_{H_{t_{1}}^{-} H_{t_{2}}^{+} Z_{\mu}}=\frac{i}{2}\left(-g_{1} s_{\Theta}+g_{2} c_{\Theta}\right)\left(Z_{t_{1} 1}^{+, *} Z_{t_{2} 1}^{+}+Z_{t_{1} 2}^{+, *} Z_{t_{2} 2}^{+}\right)
\end{aligned}
$$

\section{C.4 One scalar - two vector boson - interaction}

$$
\Gamma_{h_{t_{1}} W_{\sigma}^{+} W_{\mu}^{-}}=\frac{i}{2} g_{2}^{2}\left(v_{d} Z_{t_{1} 1}^{H}+v_{u} Z_{t_{1} 2}^{H}\right)
$$




$$
\begin{aligned}
\Gamma_{h_{1} Z_{\sigma} Z_{\mu}} & =\frac{i}{2}\left(g_{1} s_{\Theta}+g_{2} c_{\Theta}\right)^{2}\left(v_{d} Z_{t_{1} 1}^{H}+v_{u} Z_{t_{1} 2}^{H}\right) \\
\Gamma_{H_{t_{1}}^{-} W_{\sigma}^{+} \gamma_{\mu}} & =-\frac{i}{2} g_{1} g_{2}\left(v_{d} Z_{t_{1} 1}^{+, *}-v_{u} Z_{t_{1} 2}^{+, *}\right) c_{\Theta} \\
\Gamma_{H_{t_{1}}^{-} W_{\sigma}^{+} Z_{\mu}} & =\frac{i}{2} g_{1} g_{2}\left(v_{d} Z_{t_{1} 1}^{+, *}-v_{u} Z_{t_{1} 2}^{+, *}\right) s_{\Theta}
\end{aligned}
$$

\section{C.5 Two scalar - two vector boson interaction}

$$
\begin{aligned}
\Gamma_{\tilde{\nu}_{t_{1}} W_{\sigma}^{-} \tilde{\nu}_{t_{3}}^{*} W_{\nu}^{+}} & =\frac{i}{2} g_{2}^{2} \delta_{t_{1}, t_{3}} \\
\Gamma_{\tilde{\nu}_{t_{1}} Z_{\sigma} \tilde{\nu}_{t_{3}}^{*} Z_{\nu}} & =\frac{i}{2} \delta_{t_{1}, t_{3}}\left(g_{1} s_{\Theta}+g_{2} c_{\Theta}\right)^{2} \\
\Gamma_{\tilde{e}_{t_{1}} W_{\sigma}} \tilde{e}_{t_{3}}^{*} W_{\nu}^{+} & =\frac{i}{2} g_{2}^{2} \sum_{j_{2}=1}^{3} Z_{t_{1} j_{2}}^{E, *} Z_{t_{3} j_{2}}^{E} \\
\Gamma_{\tilde{e}_{t_{1}} Z_{\sigma} \tilde{e}_{t_{3}}^{*} Z_{\nu}} & =-i\left(-\frac{1}{2}\left(-g_{1} s_{\Theta}+g_{2} c_{\Theta}\right)^{2} \sum_{j_{2}=1}^{3} Z_{t_{1} j_{2}}^{E, *} Z_{t_{3} j_{2}}^{E}-2 g_{1}^{2} s_{\Theta}^{2} \sum_{j_{2}=1}^{3} Z_{t_{1} 3+j_{2}}^{E, *} Z_{t_{3} 3+j_{2}}^{E}\right) \\
\Gamma_{h_{t_{1}} W_{\sigma}} h_{t_{3} W_{\nu}} W_{\nu}^{+} & =\frac{i}{2} g_{2}^{2}\left(Z_{t_{1} 1}^{H} Z_{t_{3} 1}^{H}+Z_{t_{1} 2}^{H} Z_{t_{3} 2}^{H}\right) \\
\Gamma_{h_{t_{1}} Z_{\sigma} h_{t_{3}} Z_{\nu}} & =\frac{i}{2}\left(g_{1} s_{\Theta}+g_{2} c_{\Theta}\right)^{2}\left(Z_{t_{1} 1}^{H} Z_{t_{3} 1}^{H}+Z_{t_{1} 2}^{H} Z_{t_{3} 2}^{H}\right) \\
\Gamma_{A_{t_{1}}^{0} W_{\sigma}^{-} A_{t_{3}}^{0} W_{\nu}^{+}} & =\frac{i}{2} g_{2}^{2}\left(Z_{t_{1} 1}^{A} Z_{t_{3} 1}^{A}+Z_{t_{1} 2}^{A} Z_{t_{3} 2}^{A}\right) \\
\Gamma_{A_{t_{1}}^{0} Z_{\sigma} A_{t_{3}}^{0} Z_{\nu}} & =\frac{i}{2}\left(g_{1} s_{\Theta}+g_{2} c_{\Theta}\right)^{2}\left(Z_{t_{1} 1}^{A} Z_{t_{3} 1}^{A}+Z_{t_{1} 2}^{A} Z_{t_{3} 2}^{A}\right) \\
\Gamma_{H_{t_{1}}^{-} W_{\sigma} H_{t_{3}}^{+} W_{\nu}^{+}} & =\frac{i}{2} g_{2}^{2}\left(Z_{t_{1} 1}^{+, *} Z_{t_{3} 1}^{+}+Z_{t_{1} 2}^{+, * *} Z_{t_{3} 2}^{+}\right) \\
\Gamma_{H_{t_{1}}^{-} Z_{\sigma} H_{t_{3}}^{+} Z_{\nu}} & =\frac{i}{2}\left(-g_{1} s_{\Theta}+g_{2} c_{\Theta}\right)^{2}\left(Z_{t_{1} 1}^{+, *} Z_{t_{3} 1}^{+}+Z_{t_{1} 2}^{+, *} Z_{t_{3} 2}^{+}\right)
\end{aligned}
$$

\section{C.6 Four scalar}

We define

$$
\begin{aligned}
& A_{1}=\frac{i}{12}\left(\left(C_{L}^{1} g_{1}^{2}+3 C_{L}^{2} g_{2}^{2}\right) \sum_{j_{1}=1}^{3} Z_{t_{3} j_{1}}^{F, *} Z_{t_{1} j_{1}}^{F}+2 C_{R}^{1} g_{1}^{2} \sum_{j_{1}=1}^{3} Z_{t_{3} 3+j_{1}}^{F, *} Z_{t_{1} 3+j_{1}}^{F}\right) \\
& A_{2}=-i\left(\sum_{j_{3}=1}^{3} Z_{t_{3} 3+j_{3}}^{F, *} \sum_{j_{2}=1}^{3} \sum_{j_{1}=1}^{3} Y_{f, j_{1} j_{3}}^{*} Y_{f, j_{1} j_{2}} Z_{t_{1} 3+j_{2}}^{F}+\sum_{j_{3}=1}^{3} \sum_{j_{2}=1}^{3} Z_{t_{3} j_{2}}^{F, *} \sum_{j_{1}=1}^{3} Y_{f, j_{3} j_{1}}^{*} Y_{f, j_{2} j_{1}} Z_{t_{1} j_{3}}^{F}\right) \\
& A_{3}=\frac{i}{2}\left(\lambda \sum_{j_{2}=1}^{3} Z_{t_{3} 3+j_{2}}^{F, *} \sum_{j_{1}=1}^{3} Y_{f, j_{1} j_{2}}^{*} Z_{t_{1} j_{1}}^{F}+\lambda^{*} \sum_{j_{2}=1}^{3} \sum_{j_{1}=1}^{3} Z_{t_{3} j_{1}}^{F, *} Y_{f, j_{1} j_{2}} Z_{t_{1} 3+j_{2}}^{F}\right) \\
& A_{4}=-\frac{i}{\sqrt{2}}\left(\sum_{j_{2}=1}^{3} Z_{t_{1} 3+j_{2}}^{F, *} \sum_{j_{1}=1}^{3} T_{f, j_{1} j_{2}}^{*} Z_{t_{2} j_{1}}^{F}+\sum_{j_{2}=1}^{3} \sum_{j_{1}=1}^{3} Z_{t_{1} j_{1}}^{F, *} T_{f, j_{1} j_{2}} Z_{t_{2} 3+j_{2}}^{F}\right)
\end{aligned}
$$




$$
\begin{aligned}
A_{5}= & \frac{i}{12}\left(\left(C_{R}^{1} g_{1}^{2}+3 C_{L}^{1} g_{2}^{2}\right) \sum_{j_{1}=1}^{3} Z_{t_{1} j_{1}}^{F, *} Z_{t_{2} j_{1}}^{F}+2 g_{1}^{2} \sum_{j_{1}=1}^{3} Z_{t_{1} 3+j_{1}}^{F, *} Z_{t_{2} 3+j_{1}}^{F}\right) \\
A_{6}= & \frac{i}{2}\left(\lambda \sum_{j_{2}=1}^{3} Z_{t_{1} 3+j_{2}}^{F, *} \sum_{j_{1}=1}^{3} Y_{f, j_{1} j_{2}}^{*} Z_{t_{2} j_{1}}^{F}+\lambda^{*} \sum_{j_{2}=1}^{3} \sum_{j_{1}=1}^{3} Z_{t_{1} j_{1}}^{F, *} Y_{f, j_{1} j_{2}} Z_{t_{2} 3+j_{2}}^{F}\right) \\
A_{7}= & -i\left(\sum_{j_{3}=1}^{3} Z_{t_{1} 3+j_{3}}^{F, *} \sum_{j_{2}=1}^{3} \sum_{j_{1}=1}^{3} Y_{f, j_{1} j_{3}}^{*} Y_{f, j_{1} j_{2}} Z_{t_{2} 3+j_{2}}^{F}\right. \\
& \left.\quad+\sum_{j_{3}=1}^{3} \sum_{j_{2}=1}^{3} Z_{t_{1} j_{2}}^{F, *} \sum_{j_{1}=1}^{3} Y_{f, j_{3} j_{1}}^{*} Y_{f, j_{2} j_{1}} Z_{t_{2}}^{F} j_{3}\right) \\
A_{8}= & \frac{1}{\sqrt{2}}\left(-\sum_{j_{2}=1}^{3} Z_{t_{1} 3+j_{2}}^{F, *} \sum_{j_{1}=1}^{3} T_{f, j_{1} j_{2}}^{*} Z_{t_{2} j_{1}}^{F}+\sum_{j_{2}=1}^{3} \sum_{j_{1}=1}^{3} Z_{t_{1} j_{1}}^{F, *} T_{f, j_{1} j_{2}} Z_{t_{2} 3+j_{2}}^{F}\right) \\
A_{9}= & \frac{1}{2}\left(-\lambda \sum_{j_{2}=1}^{3} Z_{t_{1} 3+j_{2}}^{F, *} \sum_{j_{1}=1}^{3} Y_{f, j_{1} j_{2}}^{*} Z_{t_{2} j_{1}}^{F}+\lambda^{*} \sum_{j_{2}=1}^{3} \sum_{j_{1}=1}^{3} Z_{t_{1} j_{1}}^{F, *} Y_{f, j_{1} j_{2}} Z_{t_{2} 3+j_{2}}^{F}\right)
\end{aligned}
$$

With this definitions often appearing terms in the vertices involving squarks and sleptons are given by

$$
\begin{aligned}
& D_{i}=A_{i} \text { with } Y_{f} \rightarrow Y_{d}, T_{f} \rightarrow T_{d}, Z^{F} \rightarrow Z^{D}, C_{L}^{1} \rightarrow 1, C_{R}^{1} \rightarrow 1, C_{L}^{2} \rightarrow 1 \\
& U_{i}=A_{i} \text { with } Y_{f} \rightarrow Y_{u}, T_{f} \rightarrow T_{u}, Z^{F} \rightarrow Z^{U}, C_{L}^{1} \rightarrow 1, C_{R}^{1} \rightarrow-2, C_{L}^{2} \rightarrow-1 \\
& E_{i}=A_{i} \text { with } Y_{f} \rightarrow Y_{e}, T_{f} \rightarrow T_{e}, Z^{F} \rightarrow Z^{E}, C_{L}^{1} \rightarrow 3, C_{R}^{1} \rightarrow-3, C_{L}^{2} \rightarrow-1
\end{aligned}
$$

$$
\begin{aligned}
\Gamma_{\tilde{d}_{t_{1} \alpha_{1}} h_{t_{2}} \tilde{d}_{t_{3} \alpha_{3}}^{*} h_{t_{4}}=} & \delta_{\alpha_{1}, \alpha_{2}}\left(D_{1}\left(Z_{t_{2} 1}^{H} Z_{t_{4} 1}^{H}-Z_{t_{2} 2}^{H} Z_{t_{4} 2}^{H}\right)+D_{2} Z_{t_{2} 1}^{H} Z_{t_{4} 1}^{H}\right. \\
& \left.+D_{3}\left(Z_{t_{2} 2}^{H} Z_{t_{4} 3}^{H}+Z_{t_{2} 3}^{H} Z_{t_{4} 2}^{H}\right)\right) \\
\Gamma_{\tilde{d}_{t_{1} \alpha_{1}} A_{t_{2}}^{0} \tilde{t}_{t_{3} \alpha_{3}}^{*} A_{t_{4}}^{0}=} & \delta_{\alpha_{1}, \alpha_{2}}\left(D_{1}\left(Z_{t_{2} 1}^{A} Z_{t_{4} 1}^{A}-Z_{t_{2} 2}^{A} Z_{t_{4} 2}^{A}\right)+D_{2} Z_{t_{2} 1}^{A} Z_{t_{4} 1}^{A}\right. \\
& \left.+D_{3}\left(-Z_{t_{2} 2}^{A} Z_{t_{4} 3}^{A}-Z_{t_{2} 3}^{A} Z_{t_{4} 2}^{A}\right)\right) \\
\Gamma_{\tilde{u}_{t_{1} \alpha_{1}} h_{t_{2}} \tilde{u}_{t_{3} \alpha_{3}}^{*} h_{t_{4}}=}^{A} & \delta_{\alpha_{1}, \alpha_{2}}\left(U_{1}\left(Z_{t_{2} 1}^{H} Z_{t_{4} 1}^{H}-Z_{t_{2} 2}^{H} Z_{t_{4} 2}^{H}\right)+U_{2} Z_{t_{2} 2}^{H} Z_{t_{4} 2}^{H}\right. \\
& \left.+U_{3}\left(Z_{t_{2} 1}^{H} Z_{t_{4} 3}^{H}+Z_{t_{2} 3}^{H} Z_{t_{4} 1}^{H}\right)\right) \\
\Gamma_{\tilde{u}_{t_{1} \alpha_{1}} A_{t_{2}}^{0} \tilde{u}_{t_{3} \alpha_{3}}^{*} A_{t_{4}}^{0}=}^{H} & \delta_{\alpha_{1}, \alpha_{2}}\left(U_{1}\left(Z_{t_{2} 1}^{A} Z_{t_{4} 1}^{A}-Z_{t_{2} 2}^{A} Z_{t_{4} 2}^{A}\right)+U_{2} Z_{t_{2} 2}^{A} Z_{t_{4} 2}^{A}\right. \\
& \left.+U_{3}\left(-Z_{t_{2} 1}^{A} Z_{t_{4} 3}^{A}-Z_{t_{2} 3}^{A} Z_{t_{4} 1}^{A}\right)\right) \\
\Gamma_{\tilde{e}_{t_{1}} h_{t_{2}} \tilde{e}_{t_{3}}^{*} h_{t_{4}}=}^{A} & E_{1}\left(-Z_{t_{2} 1}^{H} Z_{t_{4} 1}^{H}+Z_{t_{2} 2}^{H} Z_{t_{4} 2}^{H}\right)+E_{2} Z_{t_{2} 1}^{H} Z_{t_{4} 1}^{H}+E_{3}\left(Z_{t_{2} 2}^{H} Z_{t_{4} 3}^{H}+Z_{t_{2} 3}^{H} Z_{t_{4} 2}^{H}\right) \\
\Gamma_{\tilde{e}_{t_{1}} A_{h, t_{2}} \tilde{e}_{t_{3}}^{*} A_{h, t_{4}}=}^{H} & E_{1}\left(-Z_{t_{2} 1}^{A} Z_{t_{4} 1}^{A}+Z_{t_{2} 2}^{A} Z_{t_{4} 2}^{A}\right)+E_{2} Z_{t_{2} 1}^{A} Z_{t_{4} 1}^{A}+E_{3}\left(-Z_{t_{2} 2}^{A} Z_{t_{4} 3}^{A}-Z_{t_{2} 3}^{A} Z_{t_{4} 2}^{A}\right)
\end{aligned}
$$




$$
\begin{aligned}
\Gamma_{h_{1} h_{t_{2}} h_{t_{3}} h_{t_{4}}=} & \frac{i}{4}\left(Z _ { t _ { 1 } 1 } ^ { H } \left(Z_{t_{2} 1}^{H}\left(-3 g_{+}^{2} Z_{t_{3} 1}^{H} Z_{t_{4} 1}^{H}+\tilde{\lambda} Z_{t_{3} 2}^{H} Z_{t_{4} 2}^{H}-4|\lambda|^{2} Z_{t_{3} 3}^{H} Z_{t_{4} 3}^{H}\right)\right.\right. \\
& +Z_{t_{2} 2}^{H}\left(\tilde{\lambda} Z_{t_{3} 2}^{H} Z_{t_{4} 1}^{H}+\tilde{\lambda} Z_{t_{3} 1}^{H} Z_{t_{4} 2}^{H}+4 \operatorname{Re}\{\kappa \lambda\} Z_{t_{3} 3}^{H} Z_{t_{4} 3}^{H}\right) \\
& +2 Z_{t_{2} 3}^{H}\left(\lambda \kappa^{*}\left(Z_{t_{3} 2}^{H} Z_{t_{4} 3}^{H}+Z_{t_{3} 3}^{H} Z_{t_{4} 2}^{H}\right)\right. \\
& \left.\left.+\lambda^{*}\left(\left(\kappa Z_{t_{3} 2}^{H}-2 \lambda Z_{t_{3} 1}^{H}\right) Z_{t_{4} 3}^{H}+Z_{t_{3} 3}^{H}\left(\kappa Z_{t_{4} 2}^{H}-2 \lambda Z_{t_{4} 1}^{H}\right)\right)\right)\right) \\
& +Z_{t_{1} 2}^{H}\left(Z_{t_{2} 2}^{H}\left(-3 g_{+}^{2} Z_{t_{3} 2}^{H} Z_{t_{4} 2}^{H}+\tilde{\lambda} Z_{t_{3} 1}^{H} Z_{t_{4} 1}^{H}-4|\lambda|^{2} Z_{t_{3} 3}^{H} Z_{t_{4} 3}^{H}\right)\right. \\
& +Z_{t_{2} 1}^{H}\left(\tilde{\lambda} Z_{t_{3} 2}^{H} Z_{t_{4} 1}^{H}+\tilde{\lambda} Z_{t_{3} 1}^{H} Z_{t_{4} 2}^{H}+4 \operatorname{Re}\{\kappa \lambda\} Z_{t_{3} 3}^{H} Z_{t_{4} 3}^{H}\right) \\
& +2 Z_{t_{2} 3}^{H}\left(\lambda \kappa^{*}\left(Z_{t_{3} 1}^{H} Z_{t_{4} 3}^{H}+Z_{t_{3} 3}^{H} Z_{t_{4} 1}^{H}\right)\right. \\
& \left.\left.+\lambda^{*}\left(\left(\kappa Z_{t_{3} 1}^{H}-2 \lambda Z_{t_{3} 2}^{H}\right) Z_{t_{4} 3}^{H}+Z_{t_{3} 3}^{H}\left(\kappa Z_{t_{4} 1}^{H}-2 \lambda Z_{t_{4} 2}^{H}\right)\right)\right)\right) \\
& +2 Z_{t_{1} 3}^{H}\left(\lambda ^ { * } \left(Z_{t_{2} 3}^{H}\left(Z_{t_{3} 1}^{H}\left(\kappa Z_{t_{4} 2}^{H}-2 \lambda Z_{t_{4} 1}^{H}\right)+Z_{t_{3} 2}^{H}\left(\kappa Z_{t_{4} 1}^{H}-2 \lambda Z_{t_{4} 2}^{H}\right)\right)\right.\right. \\
& +Z_{t_{2} 1}^{H}\left(\left(\kappa Z_{t_{3} 2}^{H}-2 \lambda Z_{t_{3} 1}^{H}\right) Z_{t_{4} 3}^{H}+Z_{t_{3} 3}^{H}\left(\kappa Z_{t_{4} 2}^{H}-2 \lambda Z_{t_{4} 1}^{H}\right)\right) \\
& \left.+Z_{t_{2} 2}^{H}\left(\left(\kappa Z_{t_{3} 1}^{H}-2 \lambda Z_{t_{3} 2}^{H}\right) Z_{t_{4} 3}^{H}+Z_{t_{3} 3}^{H}\left(\kappa Z_{t_{4} 1}^{H}-2 \lambda Z_{t_{4} 2}^{H}\right)\right)\right) \\
& +\kappa^{*}\left(Z_{t_{2} 3}^{H}\left(-12 \kappa Z_{t_{3} 3}^{H} Z_{t_{4} 3}^{H}+\lambda Z_{t_{3} 1}^{H} Z_{t_{4} 2}^{H}+\lambda Z_{t_{3} 2}^{H} Z_{t_{4} 1}^{H}\right)\right. \\
& \left.\left.\left.+\lambda\left(Z_{t_{2} 1}^{H}\left(Z_{t_{3} 2}^{H} Z_{t_{4} 3}^{H}+Z_{t_{3} 3}^{H} Z_{t_{4} 2}^{H}\right)+Z_{t_{2} 2}^{H}\left(Z_{t_{3} 1}^{H} Z_{t_{4} 3}^{H}+Z_{t_{3} 3}^{H} Z_{t_{4} 1}^{H}\right)\right)\right)\right)\right)
\end{aligned}
$$

$$
\begin{aligned}
\Gamma_{h_{t_{1}} h_{2} A_{t_{3}} A_{t_{4}}^{O}=} & \frac{i}{4}\left(Z _ { t _ { 3 } 2 } ^ { A } \left(Z_{t_{4} 2}^{A}\left(\tilde{\lambda} Z_{t_{1} 1}^{H} Z_{t_{2} 1}^{H}-4|\lambda|^{2} Z_{t_{1} 3}^{H} Z_{t_{2} 3}^{H}-g_{+}^{2} Z_{t_{1} 2}^{H} Z_{t_{2} 2}^{H}\right)\right.\right. \\
& \left.+2\left(\kappa \lambda^{*}+\lambda \kappa^{*}\right)\left(-Z_{t_{1} 1}^{A} Z_{t_{1} 3}^{H} Z_{t_{2} 3}^{H}+Z_{t_{4} 3}^{A}\left(Z_{t_{1} 1}^{H} Z_{t_{2} 3}^{H}+Z_{t_{1} 3}^{H} Z_{t_{2} 1}^{H}\right)\right)\right) \\
& +Z_{t_{3} 1}^{A}\left(-Z_{t_{4} 1}^{A}\left(-\tilde{\lambda} Z_{t_{1} 2}^{H} Z_{t_{2} 2}^{H}+4|\lambda|^{2} Z_{t_{1} 3}^{H} Z_{t_{2} 3}^{H}+g_{+}^{2} Z_{t_{1} 1}^{H} Z_{t_{2} 1}^{H}\right)\right. \\
& \left.+2\left(\kappa \lambda^{*}+\lambda \kappa^{*}\right)\left(-Z_{t_{4} 2}^{A} Z_{t_{1} 3}^{H} Z_{t_{2} 3}^{H}+Z_{t_{4} 3}^{A}\left(Z_{t_{1} 2}^{H} Z_{t_{2} 3}^{H}+Z_{t_{1} 3}^{H} Z_{t_{2} 2}^{H}\right)\right)\right) \\
& +2 Z_{t_{3} 3}^{A}\left(\lambda ^ { * } \left(-Z_{t_{4} 3}^{A}\left(Z_{t_{1} 1}^{H}\left(2 \lambda Z_{t_{2} 1}^{H}+\kappa Z_{t_{2} 2}^{H}\right)+Z_{t_{1} 2}^{H}\left(2 \lambda Z_{t_{2} 2}^{H}+\kappa Z_{t_{2} 1}^{H}\right)\right)\right.\right. \\
& \left.+\kappa\left(Z_{t_{4} 1}^{A}\left(Z_{t_{1} 2}^{H} Z_{t_{2} 3}^{H}+Z_{t_{1} 3}^{H} Z_{t_{2} 2}^{H}\right)+Z_{t_{4} 2}^{A}\left(Z_{t_{1} 1}^{H} Z_{t_{2} 3}^{H}+Z_{t_{1} 3}^{H} Z_{t_{2} 1}^{H}\right)\right)\right) \\
& +\kappa^{*}\left(-Z_{t_{4} 3}^{A}\left(4 \kappa Z_{t_{1} 3}^{H} Z_{t_{2} 3}^{H}+\lambda Z_{t_{1} 1}^{H} Z_{t_{2} 2}^{H}+\lambda Z_{t_{1} 2}^{H} Z_{t_{2} 1}^{H}\right)\right. \\
& \left.\left.\left.+\lambda\left(Z_{t_{4} 1}^{A}\left(Z_{t_{1} 2}^{H} Z_{t_{2} 3}^{H}+Z_{t_{1} 3}^{H} Z_{t_{2} 2}^{H}\right)+Z_{t_{4} 2}^{A}\left(Z_{t_{1} 1}^{H} Z_{t_{2} 3}^{H}+Z_{t_{1} 3}^{H} Z_{t_{2} 1}^{H}\right)\right)\right)\right)\right)
\end{aligned}
$$

$$
\begin{aligned}
\Gamma_{h_{t_{1}} H_{t_{2}}^{+} h_{t_{3} H_{t_{4}}}=} & \frac{i}{4}\left(-Z_{t_{2} 2}^{+, *}\left(4 \lambda^{*} Z_{t_{1} 3}^{H} Z_{t_{3} 3}^{H}\left(\kappa Z_{t_{4} 1}^{+}+\lambda Z_{t_{4} 2}^{+}\right)+Z_{t_{1} 1}^{H}\left(\bar{\lambda} Z_{t_{3} 2}^{H} Z_{t_{4} 1}^{+}+g_{-}^{2} Z_{t_{3} 1}^{H} Z_{t_{4} 2}^{+}\right)\right.\right. \\
& \left.+Z_{t_{1} 2}^{H}\left(\bar{\lambda} Z_{t_{3} 1}^{H} Z_{t_{4} 1}^{+}+g_{+}^{2} Z_{t_{3} 2}^{H} Z_{t_{4} 2}^{+}\right)\right)-Z_{t_{2} 1}^{+, *}\left(4 \lambda Z_{t_{1} 3}^{H} Z_{t_{3} 3}^{H}\left(\kappa^{*} Z_{t_{4} 2}^{+}+\lambda^{*} Z_{t_{4} 1}^{+}\right)\right. \\
& \left.\left.+Z_{t_{1} 2}^{H}\left(\bar{\lambda} Z_{t_{3} 1}^{H} Z_{t_{4} 2}^{+}+g_{-}^{2} Z_{t_{3} 2}^{H} Z_{t_{4} 1}^{+}\right)+Z_{t_{1} 1}^{H}\left(\bar{\lambda} Z_{t_{3} 2}^{H} Z_{t_{4} 2}^{+}+g_{+}^{2} Z_{t_{3} 1}^{H} Z_{t_{4} 1}^{+}\right)\right)\right)
\end{aligned}
$$




$$
\begin{aligned}
\Gamma_{A_{t_{1}} A_{t_{2}} A_{t_{3}}^{0} A_{t_{4}}^{0}=} & \frac{i}{4}\left(Z _ { t _ { 1 } 1 } ^ { A } \left(Z_{t_{2} 1}^{A}\left(-3 g_{+}^{2} Z_{t_{3} 1}^{A} Z_{t_{4} 1}^{A}+\tilde{\lambda} Z_{t_{3} 2}^{A} Z_{t_{4} 2}^{A}-4|\lambda|^{2} Z_{t_{3} 3}^{A} Z_{t_{4} 3}^{A}\right)\right.\right. \\
& +Z_{t_{2} 2}^{A}\left(\tilde{\lambda} Z_{t_{3} 2}^{A} Z_{t_{4} 1}^{A}+\tilde{\lambda} Z_{t_{3} 1}^{A} Z_{t_{4} 2}^{A}+4 \operatorname{Re}\{\kappa \lambda\} Z_{t_{3} 3}^{A} Z_{t_{4} 3}^{A}\right) \\
& +2 Z_{t_{2} 3}^{A}\left(\lambda \kappa^{*}\left(Z_{t_{3} 2}^{A} Z_{t_{4} 3}^{A}+Z_{t_{3} 3}^{A} Z_{t_{4} 2}^{A}\right)+\lambda^{*}\left(\left(-2 \lambda Z_{t_{3} 1}^{A}+\kappa Z_{t_{3} 2}^{A}\right) Z_{t_{4} 3}^{A}\right.\right. \\
& \left.\left.\left.+Z_{t_{3} 3}^{A}\left(-2 \lambda Z_{t_{4} 1}^{A}+\kappa Z_{t_{4} 2}^{A}\right)\right)\right)\right) \\
& +Z_{t_{1} 2}^{A}\left(Z_{t_{2} 2}^{A}\left(-3 g_{+}^{2} Z_{t_{3} 2}^{A} Z_{t_{4} 2}^{A}+\tilde{\lambda} Z_{t_{3} 1}^{A} Z_{t_{4} 1}^{A}-4|\lambda|^{2} Z_{t_{3} 3}^{A} Z_{t_{4} 3}^{A}\right)\right. \\
& +Z_{t_{2} 1}^{A}\left(\tilde{\lambda} Z_{t_{3} 2}^{A} Z_{t_{4} 1}^{A}+\tilde{\lambda} Z_{t_{3} 1}^{A} Z_{t_{4} 2}^{A}+4 \operatorname{Re}\{\kappa \lambda\} Z_{t_{3} 3}^{A} Z_{t_{4} 3}^{A}\right) \\
& +2 Z_{t_{2} 3}^{A}\left(\lambda \kappa^{*}\left(Z_{t_{3} 1}^{A} Z_{t_{4} 3}^{A}+Z_{t_{3} 3}^{A} Z_{t_{4} 1}^{A}\right)\right. \\
& \left.\left.+\lambda^{*}\left(\left(-2 \lambda Z_{t_{3} 2}^{A}+\kappa Z_{t_{3} 1}^{A}\right) Z_{t_{4} 3}^{A}+Z_{t_{3} 3}^{A}\left(\kappa Z_{t_{4} 1}^{A}-2 \lambda Z_{t_{4} 2}^{A}\right)\right)\right)\right) \\
& +2 Z_{t_{1} 3}^{A}\left(\lambda ^ { * } \left(Z_{t_{2} 3}^{A}\left(Z_{t_{3} 1}^{A}\left(-2 \lambda Z_{t_{4} 1}^{A}+\kappa Z_{t_{4} 2}^{A}\right)+Z_{t_{3} 2}^{A}\left(\kappa Z_{t_{4} 1}^{A}-2 \lambda Z_{t_{4} 2}^{A}\right)\right)\right.\right. \\
& +Z_{t_{2} 1}^{A}\left(\left(-2 \lambda Z_{t_{3} 1}^{A}+\kappa Z_{t_{3} 2}^{A}\right) Z_{t_{4} 3}^{A}+Z_{t_{3} 3}^{A}\left(-2 \lambda Z_{t_{4} 1}^{A}+\kappa Z_{t_{4} 2}^{A}\right)\right) \\
& \left.+Z_{t_{2} 2}^{A}\left(\left(-2 \lambda Z_{t_{3} 2}^{A}+\kappa Z_{t_{3} 1}^{A}\right) Z_{t_{4} 3}^{A}+Z_{t_{3} 3}^{A}\left(-2 \lambda Z_{t_{4} 2}^{A}+\kappa Z_{t_{4} 1}^{A}\right)\right)\right) \\
& +\kappa^{*}\left(Z_{t_{2} 3}^{A}\left(-12 \kappa Z_{t_{3} 3}^{A} Z_{t_{4} 3}^{A}+\lambda Z_{t_{3} 1}^{A} Z_{t_{4} 2}^{A}+\lambda Z_{t_{3} 2}^{A} Z_{t_{4} 1}^{A}\right)\right. \\
& \left.\left.\left.+\lambda\left(Z_{t_{2} 1}^{A}\left(Z_{t_{3} 2}^{A} Z_{t_{4} 3}^{A}+Z_{t_{3} 3}^{A} Z_{t_{4} 2}^{A}\right)+Z_{t_{2} 2}^{A}\left(Z_{t_{3} 1}^{A} Z_{t_{4} 3}^{A}+Z_{t_{3} 3}^{A} Z_{t_{4} 1}^{A}\right)\right)\right)\right)\right)
\end{aligned}
$$

$$
\begin{aligned}
\Gamma_{A_{t_{1}}^{0} H_{t_{2}}^{-} A_{t_{3}}^{0} H_{t_{4}}^{+}=} & \frac{i}{4}\left(-Z_{t_{2} 2}^{+, *}\left(4 \lambda^{*} Z_{t_{1} 3}^{A} Z_{t_{3} 3}^{A}\left(\lambda Z_{t_{4} 2}^{+}-\kappa Z_{t_{4} 1}^{+}\right)+Z_{t_{1} 1}^{A}\left(g_{-}^{2} Z_{t_{3} 1}^{A} Z_{t_{4} 2}^{+}-\bar{\lambda} Z_{t_{3} 2}^{A} Z_{t_{4} 1}^{+}\right)\right.\right. \\
& \left.+Z_{t_{1} 2}^{A}\left(g_{+}^{2} Z_{t_{3} 2}^{A} Z_{t_{4} 2}^{+}-\bar{\lambda} Z_{t_{3} 1}^{A} Z_{t_{4} 1}^{+}\right)\right)-Z_{t_{2} 1}^{+, *}\left(4 \lambda Z_{t_{1} 3}^{A} Z_{t_{3} 3}^{A}\left(\lambda^{*} Z_{t_{4} 1}^{+}-\kappa^{*} Z_{t_{4} 2}^{+}\right)\right. \\
& \left.\left.+Z_{t_{1} 2}^{A}\left(g_{-}^{2} Z_{t_{3} 2}^{A} Z_{t_{4} 1}^{+}-\bar{\lambda} Z_{t_{3} 1}^{A} Z_{t_{4} 2}^{+}\right)+Z_{t_{1} 1}^{A}\left(g_{+}^{2} Z_{t_{3} 1}^{A} Z_{t_{4} 1}^{+}-\bar{\lambda} Z_{t_{3} 2}^{A} Z_{t_{4} 2}^{+}\right)\right)\right)
\end{aligned}
$$

$$
\begin{aligned}
\Gamma_{H_{t_{1}}^{-} H_{t_{2}}^{-} H_{t_{3}}^{+} H_{t_{4}}^{+}=} & \frac{i}{4}\left(Z_{t_{1} 2}^{+, *}\left(-2 g_{+}^{2} Z_{t_{2} 2}^{+, *} Z_{t_{3} 2}^{+} Z_{t_{4} 2}^{+}+\tilde{\lambda} Z_{t_{2} 1}^{+, *}\left(Z_{t_{3} 1}^{+} Z_{t_{4} 2}^{+}+Z_{t_{3} 2}^{+} Z_{t_{4} 1}^{+}\right)\right)\right. \\
& \left.+Z_{t_{1} 1}^{+, *}\left(-2 g_{+}^{2} Z_{t_{2} 1}^{+, *} Z_{t_{3} 1}^{+} Z_{t_{4} 1}^{+}+\tilde{\lambda} Z_{t_{2} 2}^{+, *}\left(Z_{t_{3} 1}^{+} Z_{t_{4} 2}^{+}+Z_{t_{3} 2}^{+} Z_{t_{4} 1}^{+}\right)\right)\right)
\end{aligned}
$$

$$
\begin{aligned}
\Gamma_{\tilde{d}_{t_{1} \alpha_{1}} \tilde{\nu}_{t_{2}} \tilde{d}_{t_{3} \alpha_{3}}^{*} \tilde{\nu}_{t_{4}}^{*}=} & \frac{i}{24} \delta_{\alpha_{1}, \alpha_{3}}\left(\delta_{t_{2}, t_{4}}\left(2 g_{1}^{2} \sum_{j_{1}=1}^{3} Z_{t_{1} 3+j_{1}}^{D, *} Z_{t_{3} 3+j_{1}}^{D}+\left(3 g_{2}^{2}+g_{1}^{2}\right) \sum_{j_{1}=1}^{3} Z_{t_{1} j_{1}}^{D, *} Z_{t_{3} j_{1}}^{D}\right)\right. \\
& \left.+\delta_{t_{2}, t_{4}}\left(2 g_{1}^{2} \sum_{j_{2}=1}^{3} Z_{t_{1} 3+j_{2}}^{D, *} Z_{t_{3} 3+j_{2}}^{D}+\left(3 g_{2}^{2}+g_{1}^{2}\right) \sum_{j_{2}=1}^{3} Z_{t_{1} j_{2}}^{D, *} Z_{t_{3} j_{2}}^{D}\right)\right)
\end{aligned}
$$

$$
\Gamma_{\tilde{d}_{t_{1} \alpha_{1}} \tilde{e}_{t_{2}} \tilde{d}_{t_{3} \alpha_{3}}^{*} \tilde{e}_{t_{4}}^{*}}=-\frac{i}{24} \delta_{\alpha_{1}, \alpha_{3}}\left(2 g_{1}^{2} \sum_{j_{1}=1}^{3} Z_{t_{2} 3+j_{1}}^{E, *} Z_{t_{4} 3+j_{1}}^{E}\left(2 \sum_{j_{2}=1}^{3} Z_{t_{1} 3+j_{2}}^{D, *} Z_{t_{3} 3+j_{2}}^{D}+\sum_{j_{2}=1}^{3} Z_{t_{1} j_{2}}^{D, *} Z_{t_{3} j_{2}}^{D}\right)\right.
$$




$$
\begin{aligned}
& -\sum_{j_{1}=1}^{3} Z_{t_{2} j_{1}}^{E, *} Z_{t_{4} j_{1}}^{E}\left(2 g_{1}^{2} \sum_{j_{2}=1}^{3} Z_{t_{1} 3+j_{2}}^{D, *} Z_{t_{3} 3+j_{2}}^{D}+\left(g_{1}^{2}-3 g_{2}^{2}\right) \sum_{j_{2}=1}^{3} Z_{t_{1} j_{2}}^{D, *} Z_{t_{3} j_{2}}^{D}\right) \\
& -\left(2 g_{1}^{2} \sum_{j_{1}=1}^{3} Z_{t_{1} 3+j_{1}}^{D, *} Z_{t_{3} 3+j_{1}}^{D}+\left(g_{1}^{2}-3 g_{2}^{2}\right) \sum_{j_{1}=1}^{3} Z_{t_{1} j_{1}}^{D, *} Z_{t_{3} j_{1}}^{D}\right) \sum_{j_{2}=1}^{3} Z_{t_{2} j_{2}}^{E, *} Z_{t_{4} j_{2}}^{E} \\
& +2 g_{1}^{2}\left(2 \sum_{j_{1}=1}^{3} Z_{t_{1} 3+j_{1}}^{D, *} Z_{t_{3} 3+j_{1}}^{D}+\sum_{j_{1}=1}^{3} Z_{t_{1} j_{1}}^{D, *} Z_{t_{3} j_{1}}^{D}\right) \sum_{j_{2}=1}^{3} Z_{t_{2} 3+j_{2}}^{E, *} Z_{t_{4} 3+j_{2}}^{E} \\
& +24\left(\sum_{j_{2}=1}^{3} \sum_{j_{1}=1}^{3} Z_{t_{2} j_{1}}^{E, *} Y_{e, j_{1} j_{2}} Z_{t_{4} 3+j_{2}}^{E} \sum_{j_{4}=1}^{3} Z_{t_{1} 3+j_{4}}^{D, *} \sum_{j_{3}=1}^{3} Y_{d, j_{3} j_{4}}^{*} Z_{t_{3} j_{3}}^{D}\right. \\
& \left.\left.+\sum_{j_{2}=1}^{3} \sum_{j_{1}=1}^{3} Z_{t_{1} j_{1}}^{D, *} Y_{d, j_{1} j_{2}} Z_{t_{3} 3+j_{2}}^{D} \sum_{j_{4}=1}^{3} Z_{t_{2} 3+j_{4}}^{E, *} \sum_{j_{3}=1}^{3} Y_{e, j_{3} j_{4}}^{*} Z_{t_{4} j_{3}}^{E}\right)\right) \\
& \Gamma_{\tilde{d}_{t_{1} \alpha_{1}} H_{t_{2}}^{-} \tilde{d}_{t_{3} \alpha_{3}}^{*} H_{t_{4}}^{+}}=\frac{i}{12} \delta_{\alpha_{1}, \alpha_{3}}\left(Z _ { t _ { 2 } 1 } ^ { + , * } \left(\left(g_{1}^{2}-3 g_{2}^{2}\right) \sum_{j_{1}=1}^{3} Z_{t_{1} j_{1}}^{D, *} Z_{t_{3} j_{1}}^{D}+2 g_{1}^{2} \sum_{j_{1}=1}^{3} Z_{t_{1} 3+j_{1}}^{D, *} Z_{t_{3} 3+j_{1}}^{D}\right.\right. \\
& \left.-12 \sum_{j_{3}=1}^{3} Z_{t_{1} 3+j_{3}}^{D, *} \sum_{j_{2}=1}^{3} \sum_{j_{1}=1}^{3} Y_{d, j_{1} j_{3}}^{*} Y_{d, j_{1} j_{2}} Z_{t_{3} 3+j_{2}}^{D}\right) Z_{t_{4} 1}^{+} \\
& -Z_{t_{2} 2}^{+, *}\left(\left(g_{1}^{2}-3 g_{2}^{2}\right) \sum_{j_{1}=1}^{3} Z_{t_{1} j_{1}}^{D, *} Z_{t_{3} j_{1}}^{D}+2 g_{1}^{2} \sum_{j_{1}=1}^{3} Z_{t_{1} 3+j_{1}}^{D, *} Z_{t_{3} 3+j_{1}}^{D}\right. \\
& \left.\left.+12 \sum_{j_{3}=1}^{3} \sum_{j_{2}=1}^{3} Z_{t_{1} j_{2}}^{D, *} \sum_{j_{1}=1}^{3} Y_{u, j_{3} j_{1}}^{*} Y_{u, j_{2} j_{1}} Z_{t_{3} j_{3}}^{D}\right) Z_{t_{4} 2}^{+}\right) \\
& \Gamma_{\tilde{\nu}_{t_{1}} \tilde{\nu}_{t_{2}} \tilde{\nu}_{t_{3}}^{*} \tilde{\nu}_{t_{4}}^{*}}=-\frac{i}{8}\left(g_{1}^{2}+g_{2}^{2}\right)\left(2 \delta_{t_{1}, t_{3}} \delta_{t_{2}, t_{4}}+2 \delta_{t_{1}, t_{4}} \delta_{t_{2}, t_{3}}\right) \\
& \Gamma_{\tilde{\nu}_{t_{1}} \tilde{u}_{t_{2} \alpha_{2}} \tilde{\nu}_{t_{3}}^{*} \tilde{u}_{t_{4} \alpha_{4}}^{*}}=\frac{i}{24} \delta_{\alpha_{2}, \alpha_{4}}\left(\delta_{t_{1}, t_{3}}\left(\left(-3 g_{2}^{2}+g_{1}^{2}\right) \sum_{j_{1}=1}^{3} Z_{t_{2} j_{1}}^{U, *} Z_{t_{4} j_{1}}^{U}-4 g_{1}^{2} \sum_{j_{1}=1}^{3} Z_{t_{2} 3+j_{1}}^{U, *} Z_{t_{4} 3+j_{1}}^{U}\right)\right. \\
& \left.+\delta_{t_{1}, t_{3}}\left(\left(-3 g_{2}^{2}+g_{1}^{2}\right) \sum_{j_{2}=1}^{3} Z_{t_{2} j_{2}}^{U, *} Z_{t_{4} j_{2}}^{U}-4 g_{1}^{2} \sum_{j_{2}=1}^{3} Z_{t_{2} 3+j_{2}}^{U, *} Z_{t_{4} 3+j_{2}}^{U}\right)\right)
\end{aligned}
$$

$$
\begin{aligned}
\Gamma_{\tilde{\nu}_{t_{1}} \tilde{e}_{t_{2}} \tilde{\nu}_{t_{3}}^{*} \tilde{e}_{t_{4}}^{*}=} & -\frac{i}{4}\left(\delta_{t_{1}, t_{3}}\left(\left(g_{1}^{2}-g_{2}^{2}\right) \sum_{j_{1}=1}^{3} Z_{t_{2} j_{1}}^{E, *} Z_{t_{4} j_{1}}^{E}-2 g_{1}^{2} \sum_{j_{1}=1}^{3} Z_{t_{2} 3+j_{1}}^{E, *} Z_{t_{4} 3+j_{1}}^{E}\right)\right. \\
& +g_{2}^{2}\left(\sum_{j_{1}=1}^{3} Z_{t_{2} j_{1}}^{E, *} Z_{t_{3} j_{1}}^{\nu} \sum_{j_{2}=1}^{3} Z_{t_{1} j_{2}}^{\nu, *} Z_{t_{4} j_{2}}^{E}+\sum_{j_{1}=1}^{3} Z_{t_{1} j_{1}}^{\nu, *} Z_{t_{4} j_{1}}^{E} \sum_{j_{2}=1}^{3} Z_{t_{2} j_{2}}^{E, *} Z_{t_{3} j_{2}}^{\nu}\right) \\
& \left.+4 \sum_{j_{2}=1}^{3} \sum_{j_{1}=1}^{3} Z_{t_{1} j_{1}}^{\nu, *} Y_{e, j_{1} j_{2}} Z_{t_{4} 3+j_{2}}^{E} \sum_{j_{4}=1}^{3} Z_{t_{2} 3+j_{4}}^{E, *} \sum_{j_{3}=1}^{3} Y_{e, j_{3} j_{4}}^{*} Z_{t_{3} j_{3}}^{\nu}\right) \\
\Gamma_{\tilde{\nu}_{t_{1}} h_{t_{2}} \tilde{\nu}_{t_{3}}^{*} h_{t_{4}}}= & -\frac{i}{4}\left(g_{1}^{2}+g_{2}^{2}\right) \delta_{t_{1}, t_{3}}\left(Z_{t_{2} 1}^{H} Z_{t_{4} 1}^{H}-Z_{t_{2} 2}^{H} Z_{t_{4} 2}^{H}\right)
\end{aligned}
$$




$$
\begin{aligned}
\Gamma_{\tilde{\nu}_{t_{1}} A_{t_{2}}^{0} \tilde{\nu}_{t_{3}}^{*} A_{t_{4}}^{0}=} & -\frac{i}{4}\left(g_{1}^{2}+g_{2}^{2}\right) \delta_{t_{1}, t_{3}}\left(Z_{t_{2} 1}^{A} Z_{t_{4} 1}^{A}-Z_{t_{2} 2}^{A} Z_{t_{4} 2}^{A}\right) \\
\Gamma_{\tilde{\nu}_{t_{1}} H_{t_{2}} \tilde{\nu}_{t_{3}} \tilde{H}_{t_{4}}^{+}=} & \frac{i}{4}\left(Z_{t_{2} 1}^{+, *}\left(\left(g_{2}^{2}-g_{1}^{2}\right) \delta_{t_{1}, t_{3}}-4 \sum_{j_{3}=1}^{3} \sum_{j_{2}=1}^{3} Z_{t_{1} j_{2}}^{\nu, *} \sum_{j_{1}=1}^{3} Y_{e, j_{3} j_{1}}^{*} Y_{e, j_{2} j_{1}} Z_{t_{3} j_{3}}^{\nu}\right) Z_{t_{4} 1}^{+}\right. \\
& \left.+\left(g_{1}^{2}-g_{2}^{2}\right) Z_{t_{2} 2}^{+, *} \delta_{t_{1}, t_{3}} Z_{t_{4} 2}^{+}\right) \\
\Gamma_{\tilde{u}_{t_{1} \alpha_{1}} \tilde{e}_{t_{2}} \tilde{u}_{t_{3} \alpha_{3}}^{*} \tilde{e}_{t_{4}}^{*}=} & \frac{i}{24} \delta_{\alpha_{1}, \alpha_{3}}\left(-4 g_{1}^{2} \sum_{j_{1}=1}^{3} Z_{t_{1} 3+j_{1}}^{U, *} Z_{t_{3} 3+j_{1}}^{U}\left(\sum_{j_{2}=1}^{3} Z_{t_{2} j_{2}}^{E, *} Z_{t_{4} j_{2}}^{E}-2 \sum_{j_{2}=1}^{3} Z_{t_{2} 3+j_{2}}^{E, *} Z_{t_{4} 3+j_{2}}^{E}\right)\right. \\
& +\sum_{j_{1}=1}^{3} Z_{t_{1} j_{1}}^{U, *} Z_{t_{3} j_{1}}^{U}\left(\left(3 g_{2}^{2}+g_{1}^{2}\right) \sum_{j_{2}=1}^{3} Z_{t_{2} j_{2}}^{E, *} Z_{t_{4} j_{2}}^{E}-2 g_{1}^{2} \sum_{j_{2}=1}^{3} Z_{t_{2} 3+j_{2}}^{E, *} Z_{t_{4} 3+j_{2}}^{E}\right) \\
& +\left(\left(3 g_{2}^{2}+g_{1}^{2}\right) \sum_{j_{1}=1}^{3} Z_{t_{2} j_{1}}^{E, *} Z_{t_{4} j_{1}}^{E}-2 g_{1}^{2} \sum_{j_{1}=1}^{3} Z_{t_{2} 3+j_{1}}^{E, *} Z_{t_{4} 3+j_{1}}^{E}\right) \sum_{j_{2}=1}^{3} Z_{t_{1} j_{2}}^{U, *} Z_{t_{3} j_{2}}^{U} \\
& \left.-4 g_{1}^{2}\left(\sum_{j_{1}=1}^{3} Z_{t_{2} j_{1}}^{E, *} Z_{t_{4} j_{1}}^{E}-2 \sum_{j_{1}=1}^{3} Z_{t_{2} 3+j_{1}}^{E, *} Z_{t_{4} 3+j_{1}}^{E}\right) \sum_{j_{2}=1}^{3} Z_{t_{1} 3+j_{2}}^{U, *} Z_{t_{3} 3+j_{2}}^{U}\right)
\end{aligned}
$$

$$
\begin{aligned}
\Gamma_{\tilde{u}_{t_{1} \alpha_{1}} H_{t_{2}} \tilde{u}_{t_{3} \alpha_{3}}^{*} H_{t_{4}}^{+}=} & \frac{i}{12} \delta_{\alpha_{1}, \alpha_{3}}\left(Z _ { t _ { 2 } 1 } ^ { + , * } \left(\left(3 g_{2}^{2}+g_{1}^{2}\right) \sum_{j_{1}=1}^{3} Z_{t_{1} j_{1}}^{U, *} Z_{t_{3} j_{1}}^{U}-4\left(g_{1}^{2} \sum_{j_{1}=1}^{3} Z_{t_{1} 3+j_{1}}^{U, *} Z_{t_{3} 3+j_{1}}^{U}\right.\right.\right. \\
& \left.\left.+3 \sum_{j_{3}=1}^{3} \sum_{j_{2}=1}^{3} Z_{t_{1} j_{2}}^{U, *} \sum_{j_{1}=1}^{3} Y_{d, j_{3} j_{1}}^{*} Y_{d, j_{2} j_{1}} Z_{t_{3} j_{3}}^{U}\right)\right) Z_{t_{4} 1}^{+}
\end{aligned}
$$$$
-Z_{t_{2} 2}^{+, *}\left(\left(3 g_{2}^{2}+g_{1}^{2}\right) \sum_{j_{1}=1}^{3} Z_{t_{1} j_{1}}^{U, *} Z_{t_{3} j_{1}}^{U}-4 g_{1}^{2} \sum_{j_{1}=1}^{3} Z_{t_{1} 3+j_{1}}^{U, *} Z_{t_{3} 3+j_{1}}^{U}\right.
$$$$
\left.\left.+12 \sum_{j_{3}=1}^{3} Z_{t_{1} 3+j_{3}}^{U, *} \sum_{j_{2}=1}^{3} \sum_{j_{1}=1}^{3} Y_{u, j_{1} j_{3}}^{*} Y_{u, j_{1} j_{2}} Z_{t_{3} 3+j_{2}}^{U}\right) Z_{t_{4} 2}^{+}\right)
$$$$
\Gamma_{\tilde{e}_{t_{1}} \tilde{e}_{t_{2}}} \tilde{e}_{t_{3}}^{*} \tilde{e}_{t_{4}}^{*}=-\frac{i}{8}\left(g_{1}^{2} \sum_{j_{1}=1}^{3} Z_{t_{1} j_{1}}^{E, *} Z_{t_{4} j_{1}}^{E} \sum_{j_{2}=1}^{3} Z_{t_{2} j_{2}}^{E, *} Z_{t_{3} j_{2}}^{E}+g_{2}^{2} \sum_{j_{1}=1}^{3} Z_{t_{1} j_{1}}^{E, *} Z_{t_{4} j_{1}}^{E} \sum_{j_{2}=1}^{3} Z_{t_{2} j_{2}}^{E, *} Z_{t_{3} j_{2}}^{E}\right.
$$$$
-2 g_{1}^{2} \sum_{j_{1}=1}^{3} Z_{t_{1} 3+j_{1}}^{E, *} Z_{t_{4} 3+j_{1}}^{E} \sum_{j_{2}=1}^{3} Z_{t_{2} j_{2}}^{E, *} Z_{t_{3} j_{2}}^{E}
$$$$
-2 g_{1}^{2} \sum_{j_{1}=1}^{3} Z_{t_{2} 3+j_{1}}^{E, *} Z_{t_{4} 3+j_{1}}^{E}\left(\sum_{j_{2}=1}^{3} Z_{t_{1} j_{2}}^{E, *} Z_{t_{3} j_{2}}^{E}-2 \sum_{j_{2}=1}^{3} Z_{t_{1} 3+j_{2}}^{E, *} Z_{t_{3} 3+j_{2}}^{E}\right)
$$$$
+\sum_{j_{1}=1}^{3} Z_{t_{2} j_{1}}^{E, *} Z_{t_{4} j_{1}}^{E}\left(\left(g_{1}^{2}+g_{2}^{2}\right) \sum_{j_{2}=1}^{3} Z_{t_{1} j_{2}}^{E, *} Z_{t_{3} j_{2}}^{E}-2 g_{1}^{2} \sum_{j_{2}=1}^{3} Z_{t_{1} 3+j_{2}}^{E, *} Z_{t_{3} 3+j_{2}}^{E}\right)
$$$$
-2 g_{1}^{2}\left(\sum_{j_{1}=1}^{3} Z_{t_{1} j_{1}}^{E, *} Z_{t_{4} j_{1}}^{E} \sum_{j_{2}=1}^{3} Z_{t_{2} 3+j_{2}}^{E, *} Z_{t_{3} 3+j_{2}}^{E}+\sum_{j_{1}=1}^{3} Z_{t_{2} j_{1}}^{E, *} Z_{t_{3} j_{1}}^{E} \sum_{j_{2}=1}^{3} Z_{t_{1} 3+j_{2}}^{E, *} Z_{t_{4} 3+j_{2}}^{E}\right)
$$ 


$$
\begin{aligned}
& +4 g_{1}^{2}\left(\sum_{j_{1}=1}^{3} Z_{t_{1} 3+j_{1}}^{E, *} Z_{t_{4} 3+j_{1}}^{E} \sum_{j_{2}=1}^{3} Z_{t_{2} 3+j_{2}}^{E, *} Z_{t_{3} 3+j_{2}}^{E}\right. \\
& \left.+\sum_{j_{1}=1}^{3} Z_{t_{2} 3+j_{1}}^{E, *} Z_{t_{3} 3+j_{1}}^{E} \sum_{j_{2}=1}^{3} Z_{t_{1} 3+j_{2}}^{E, *} Z_{t_{4} 3+j_{2}}^{E}\right) \\
& +g_{1}^{2} \sum_{j_{1}=1}^{3} Z_{t_{2} j_{1}}^{E, *} Z_{t_{3} j_{1}}^{E} \sum_{j_{2}=1}^{3} Z_{t_{1} j_{2}}^{E, *} Z_{t_{4} j_{2}}^{E}+g_{2}^{2} \sum_{j_{1}=1}^{3} Z_{t_{2} j_{1}}^{E, *} Z_{t_{3} j_{1}}^{E} \sum_{j_{2}=1}^{3} Z_{t_{1} j_{2}}^{E, *} Z_{t_{4} j_{2}}^{E} \\
& -2 g_{1}^{2} \sum_{j_{1}=1}^{3} Z_{t_{2} 3+j_{1}}^{E, *} Z_{t_{3} 3+j_{1}}^{E} \sum_{j_{2}=1}^{3} Z_{t_{1} j_{2}}^{E, *} Z_{t_{4} j_{2}}^{E}+g_{1}^{2} \sum_{j_{1}=1}^{3} Z_{t_{1} j_{1}}^{E, *} Z_{t_{3} j_{1}}^{E} \sum_{j_{2}=1}^{3} Z_{t_{2} j_{2}}^{E, *} Z_{t_{4} j_{2}}^{E} \\
& +g_{2}^{2} \sum_{j_{1}=1}^{3} Z_{t_{1} j_{1}}^{E, *} Z_{t_{3} j_{1}}^{E} \sum_{j_{2}=1}^{3} Z_{t_{2} j_{2}}^{E, *} Z_{t_{4} j_{2}}^{E}-2 g_{1}^{2} \sum_{j_{1}=1}^{3} Z_{t_{1} 3+j_{1}}^{E, *} Z_{t_{3} 3+j_{1}}^{E} \sum_{j_{2}=1}^{3} Z_{t_{2} j_{2}}^{E, *} Z_{t_{4} j_{2}}^{E} \\
& 2 g_{1}^{2}\left(\sum_{j_{1}=1}^{3} Z_{t_{1} j_{1}}^{E, *} Z_{t_{3} j_{1}}^{E}-2 \sum_{j_{1}=1}^{3} Z_{t_{1} 3+j_{1}}^{E, *} Z_{t_{3} 3+j_{1}}^{E}\right) \sum_{j_{2}=1}^{3} Z_{t_{2} 3+j_{2}}^{E, *} Z_{t_{4} 3+j_{2}}^{E} \\
& +8\left(\sum_{j_{2}=1}^{3} \sum_{j_{1}=1}^{3} Z_{t_{2} j_{1}}^{E, *} Y_{e, j_{1} j_{2}} Z_{t_{4} 3+j_{2}}^{E} \sum_{j_{4}=1}^{3} Z_{t_{1} 3+j_{4}}^{E, *} \sum_{j_{3}=1}^{3} Y_{e, j_{3} j_{4}}^{*} Z_{t_{3} j_{3}}^{E}\right. \\
& +\sum_{j_{2}=1}^{3} \sum_{j_{1}=1}^{3} Z_{t_{1} j_{1}}^{E, *} Y_{e, j_{1} j_{2}} Z_{t_{4} 3+j_{2}}^{E} \sum_{j_{4}=1}^{3} Z_{t_{2} 3+j_{4}}^{E, *} \sum_{j_{3}=1}^{3} Y_{e, j_{3} j_{4}}^{*} Z_{t_{3} j_{3}}^{E} \\
& +\sum_{j_{2}=1}^{3} \sum_{j_{1}=1}^{3} Z_{t_{2} j_{1}}^{E, *} Y_{e, j_{1} j_{2}} Z_{t_{3} 3+j_{2}}^{E} \sum_{j_{4}=1}^{3} Z_{t_{1} 3+j_{4}}^{E, *} \sum_{j_{3}=1}^{3} Y_{e, j_{3} j_{4}}^{*} Z_{t_{4} j_{3}}^{E} \\
& \left.\left.+\sum_{j_{2}=1}^{3} \sum_{j_{1}=1}^{3} Z_{t_{1} j_{1}}^{E, *} Y_{e, j_{1} j_{2}} Z_{t_{3} 3+j_{2}}^{E} \sum_{j_{4}=1}^{3} Z_{t_{2} 3+j_{4}}^{E, *} \sum_{j_{3}=1}^{3} Y_{e, j_{3} j_{4}}^{*} Z_{t_{4} j_{3}}^{E}\right)\right) \\
& \Gamma_{\tilde{e}_{t_{1}} H_{t_{2}}^{-}} \tilde{e}_{t_{3}}^{*} H_{t_{4}}^{+}=\frac{i}{4}\left(-Z_{t_{2} 1}^{+, *}\left(\left(g_{1}^{2}+g_{2}^{2}\right) \sum_{j_{1}=1}^{3} Z_{t_{1} j_{1}}^{E, *} Z_{t_{3} j_{1}}^{E}-2 g_{1}^{2} \sum_{j_{1}=1}^{3} Z_{t_{1} 3+j_{1}}^{E, *} Z_{t_{3} 3+j_{1}}^{E}\right.\right. \\
& \left.+4 \sum_{j_{3}=1}^{3} Z_{t_{1} 3+j_{3}}^{E, *} \sum_{j_{2}=1}^{3} \sum_{j_{1}=1}^{3} Y_{e, j_{1} j_{3}}^{*} Y_{e, j_{1} j_{2}} Z_{t_{3} 3+j_{2}}^{E}\right) Z_{t_{4} 1}^{+} \\
& \left.+Z_{t_{2} 2}^{+, *}\left(-2 g_{1}^{2} \sum_{j_{1}=1}^{3} Z_{t_{1} 3+j_{1}}^{E, *} Z_{t_{3} 3+j_{1}}^{E}+\left(g_{1}^{2}+g_{2}^{2}\right) \sum_{j_{1}=1}^{3} Z_{t_{1} j_{1}}^{E, *} Z_{t_{3} j_{1}}^{E}\right) Z_{t_{4} 2}^{+}\right)
\end{aligned}
$$

\section{C.7 Three scalar}

$$
\begin{aligned}
\Gamma_{\tilde{d}_{t_{1} \alpha_{1}} \tilde{d}_{t_{2} \alpha_{2}}^{*} h_{t_{3}}} & =\delta_{\alpha_{1}, \alpha_{2}}\left(D_{4} Z_{t_{3} 1}^{H}+D_{7} v_{d} Z_{t_{3} 1}^{H}+D_{5}\left(v_{d} Z_{t_{3} 1}^{H}-v_{u} Z_{t_{3} 2}^{H}\right)+D_{6}\left(v_{s} Z_{t_{3} 2}^{H}+v_{u} Z_{t_{3} 3}^{H}\right)\right) \\
\Gamma_{\tilde{d}_{t_{1} \alpha_{1}} \tilde{d}_{t_{2} \alpha_{2}}^{*} A_{h^{*}, t_{3}}}^{H} & =\delta_{\alpha_{1}, \alpha_{2}}\left(D_{8} Z_{t_{3} 1}^{A}+D_{9}\left(v_{s} Z_{t_{3} 2}^{A}+v_{u} Z_{t_{3}}^{A}\right)\right)
\end{aligned}
$$




$$
\begin{aligned}
& \Gamma_{\tilde{u}_{t_{1} \alpha_{1}} \tilde{u}_{t_{2} \alpha_{2}}^{*} h_{t_{3}}}=\delta_{\alpha_{1}, \alpha_{2}}\left(U_{4} Z_{t_{3} 2}^{H}+U_{7} v_{u} Z_{t_{3} 2}^{H}+U_{5}\left(v_{d} Z_{t_{3} 1}^{H}-v_{u} Z_{t_{3} 2}^{H}\right)+U_{6}\left(v_{d} Z_{t_{3} 3}^{H}+v_{s} Z_{t_{3} 1}^{H}\right)\right) \\
& \Gamma_{\tilde{u}_{t_{1} \alpha_{1}} \tilde{u}_{t_{2} \alpha_{2}}^{*} A_{t_{3}}^{0}}=\delta_{\alpha_{1}, \alpha_{2}}\left(U_{8} Z_{t_{3} 2}^{A}+U_{9}\left(v_{d} Z_{t_{3} 3}^{A}+v_{s} Z_{t_{3} 1}^{A}\right)\right) \\
& \Gamma_{\tilde{e}_{t_{1}} \tilde{e}_{t_{2}}^{*} h_{t_{3}}}=E_{4} Z_{t_{3} 1}^{H}+E_{7} v_{d} Z_{t_{3} 1}^{H}+E_{5}\left(-v_{d} Z_{t_{3} 1}^{H}+v_{u} Z_{t_{3} 2}^{H}\right)+E_{6}\left(v_{s} Z_{t_{3} 2}^{H}+v_{u} Z_{t_{3} 3}^{H}\right) \\
& \Gamma_{\tilde{e}_{t_{1}} \tilde{e}_{t_{2}}^{*} A_{t_{3}}^{0}}=E_{8} Z_{t_{3} 1}^{A}+E_{9}\left(v_{s} Z_{t_{3} 2}^{A}+v_{u} Z_{t_{3} 3}^{A}\right) \\
& \Gamma_{\tilde{u}_{t_{1} \alpha_{1}} \tilde{d}_{t_{2} \alpha_{2}}^{*} H_{t_{3}}^{-}}=-\frac{i}{4} \delta_{\alpha_{1}, \alpha_{2}}\left(Z _ { t _ { 3 } 1 } ^ { + , * } \left(\sqrt{2} g_{2}^{2} v_{d} \sum_{j_{1}=1}^{3} Z_{t_{1} j_{1}}^{U, *} Z_{t_{2} j_{1}}^{D}\right.\right. \\
& -2\left(\sqrt{2} v_{s} \lambda \sum_{j_{2}=1}^{3} Z_{t_{1} 3+j_{2}}^{U, *} \sum_{j_{1}=1}^{3} Y_{u, j_{1} j_{2}}^{*} Z_{t_{2} j_{1}}^{D}+2 \sum_{j_{2}=1}^{3} \sum_{j_{1}=1}^{3} Z_{t_{1} j_{1}}^{U, *} T_{d, j_{1} j_{2}} Z_{t_{2} 3+j_{2}}^{D}\right. \\
& +\sqrt{2}\left(v_{u} \sum_{j_{3}=1}^{3} Z_{t_{1} 3+j_{3}}^{U, *} \sum_{j_{2}=1}^{3} \sum_{j_{1}=1}^{3} Y_{u, j_{1} j_{3}}^{*} Y_{d, j_{1} j_{2}} Z_{t_{2} 3+j_{2}}^{D}\right. \\
& \left.\left.\left.+v_{d} \sum_{j_{3}=1}^{3} \sum_{j_{2}=1}^{3} Z_{t_{1} j_{2}}^{U, *} \sum_{j_{1}=1}^{3} Y_{d, j_{3} j_{1}}^{*} Y_{d, j_{2} j_{1}} Z_{t_{2} j_{3}}^{D}\right)\right)\right)+Z_{t_{3} 2}^{+, *}\left(\sqrt{2} g_{2}^{2} v_{u} \sum_{j_{1}=1}^{3} Z_{t_{1} j_{1}}^{U, *} Z_{t_{2} j_{1}}^{D}\right. \\
& -2\left(2 \sum_{j_{2}=1}^{3} Z_{t_{1} 3+j_{2}}^{U, *} \sum_{j_{1}=1}^{3} T_{u, j_{1} j_{2}}^{*} Z_{t_{2} j_{1}}^{D}+\sqrt{2}\left(v_{s} \lambda^{*} \sum_{j_{2}=1}^{3} \sum_{j_{1}=1}^{3} Z_{t_{1} j_{1}}^{U, *} Y_{d, j_{1} j_{2}} Z_{t_{2} 3+j_{2}}^{D}\right.\right. \\
& +v_{d} \sum_{j_{3}=1}^{3} Z_{t_{1} 3+j_{3}}^{U, *} \sum_{j_{2}=1}^{3} \sum_{j_{1}=1}^{3} Y_{u, j_{1} j_{3}}^{*} Y_{d, j_{1} j_{2}} Z_{t_{2} 3+j_{2}}^{D} \\
& \left.\left.\left.\left.+v_{u} \sum_{j_{3}=1}^{3} \sum_{j_{2}=1}^{3} Z_{t_{1} j_{2}}^{U, *} \sum_{j_{1}=1}^{3} Y_{u, j_{3} j_{1}}^{*} Y_{u, j_{2} j_{1}} Z_{t_{2} j_{3}}^{D}\right)\right)\right)\right) \\
& \Gamma_{\tilde{\nu}_{t_{1}} \tilde{\nu}_{t_{2}}^{*} h_{t_{3}}}=-\frac{i}{4}\left(g_{1}^{2}+g_{2}^{2}\right) \delta_{t_{1}, t_{2}}\left(v_{d} Z_{t_{3} 1}^{H}-v_{u} Z_{t_{3} 2}^{H}\right) \\
& \Gamma_{\tilde{\nu}_{t_{1}} \tilde{e}_{t_{2}}^{*} H_{t_{3}}^{-}}=\frac{i}{4}\left(\sqrt{2} Z_{t_{3} 2}^{+, *}\left(-g_{2}^{2} v_{u} \sum_{j_{1}=1}^{3} Z_{t_{1} j_{1}}^{\nu, *} Z_{t_{2} j_{1}}^{E}+2 v_{s} \lambda^{*} \sum_{j_{2}=1}^{3} \sum_{j_{1}=1}^{3} Z_{t_{1} j_{1}}^{\nu, *} Y_{e, j_{1} j_{2}} Z_{t_{2} 3+j_{2}}^{E}\right)\right. \\
& +Z_{t_{3} 1}^{+, *}\left(-\sqrt{2} g_{2}^{2} v_{d} \sum_{j_{1}=1}^{3} Z_{t_{1} j_{1}}^{\nu, *} Z_{t_{2} j_{1}}^{E}+4 \sum_{j_{2}=1}^{3} \sum_{j_{1}=1}^{3} Z_{t_{1} j_{1}}^{\nu, *} T_{e, j_{1} j_{2}} Z_{t_{2} 3+j_{2}}^{E}\right. \\
& \left.\left.+2 \sqrt{2} v_{d} \sum_{j_{3}=1}^{3} \sum_{j_{2}=1}^{3} Z_{t_{1} j_{2}}^{\nu, *} \sum_{j_{1}=1}^{3} Y_{e, j_{3} j_{1}}^{*} Y_{e, j_{2} j_{1}} Z_{t_{2} j_{3}}^{E}\right)\right) \\
& \Gamma_{h_{t_{1}} h_{t_{2}} h_{t_{3}}}=\frac{i}{4}\left(Z _ { t _ { 1 } 1 } ^ { H } \left(Z_{t_{2} 1}^{H}\left(-3 g_{+}^{2} v_{d} Z_{t_{3} 1}^{H}-4 v_{s}|\lambda|^{2} Z_{t_{3} 3}^{H}+v_{u} \tilde{\lambda} Z_{t_{3} 2}^{H}\right)\right.\right. \\
& +Z_{t_{2} 2}^{H}\left(v_{u} \tilde{\lambda} Z_{t_{3} 1}^{H}+v_{d} \tilde{\lambda} Z_{t_{3} 2}^{H}+\Lambda_{1} Z_{t_{3} 3}^{H}\right) \\
& +Z_{t_{2} 3}^{H}\left(\sqrt{2} 2 \operatorname{Re}\left\{T_{\lambda}\right\} Z_{t_{3} 2}^{H}+2 \lambda \kappa^{*}\left(v_{s} Z_{t_{3} 2}^{H}+v_{u} Z_{t_{3} 3}^{H}\right)\right. \\
& \left.\left.+2 \lambda^{*}\left(\Lambda_{3} Z_{t_{3} 3}^{H}-2 v_{s} \lambda Z_{t_{3} 1}^{H}+v_{s} \kappa Z_{t_{3} 2}^{H}\right)\right)\right)
\end{aligned}
$$




$$
\begin{aligned}
& +Z_{t_{1} 2}^{H}\left(Z_{t_{2} 2}^{H}\left(-3 g_{+}^{2} v_{u} Z_{t_{3} 2}^{H}-4 v_{s}|\lambda|^{2} Z_{t_{3} 3}^{H}+v_{d} \tilde{\lambda} Z_{t_{3} 1}^{H}\right)\right. \\
& +Z_{t_{2} 1}^{H}\left(v_{u} \tilde{\lambda} Z_{t_{3} 1}^{H}+v_{d} \tilde{\lambda} Z_{t_{3} 2}^{H}+\Lambda_{1} Z_{t_{3} 3}^{H}\right) \\
& +Z_{t_{2} 3}^{H}\left(\sqrt{2} \operatorname{Re}\left\{T_{\lambda}\right\} Z_{t_{3} 1}^{H}+2 \lambda \kappa^{*}\left(v_{d} Z_{t_{3} 3}^{H}+v_{s} Z_{t_{3} 1}^{H}\right)\right. \\
& \left.\left.+2 \lambda^{*}\left(-2 v_{s} \lambda Z_{t_{3} 2}^{H}+\Lambda_{2} Z_{t_{3} 3}^{H}+v_{s} \kappa Z_{t_{3} 1}^{H}\right)\right)\right) \\
& +Z_{t_{1} 3}^{H}\left(\sqrt { 2 } \left(-4 \operatorname{Re}\left\{T_{\kappa}\right\} Z_{t_{2} 3}^{H} Z_{t_{3} 3}^{H}+T_{\lambda}^{*}\left(Z_{t_{2} 1}^{H} Z_{t_{3} 2}^{H}+Z_{t_{2} 2}^{H} Z_{t_{3} 1}^{H}\right)\right.\right. \\
& \left.+T_{\lambda}\left(Z_{t_{2} 1}^{H} Z_{t_{3} 2}^{H}+Z_{t_{2} 2}^{H} Z_{t_{3} 1}^{H}\right)\right)+2 \kappa^{*}\left(\lambda Z_{t_{2} 2}^{H}\left(v_{d} Z_{t_{3} 3}^{H}+v_{s} Z_{t_{3} 1}^{H}\right)\right. \\
& \left.+\lambda Z_{t_{2} 1}^{H}\left(v_{s} Z_{t_{3} 2}^{H}+v_{u} Z_{t_{3} 3}^{H}\right)+Z_{t_{2} 3}^{H}\left(-12 v_{s} \kappa Z_{t_{3} 3}^{H}+v_{d} \lambda Z_{t_{3} 2}^{H}+v_{u} \lambda Z_{t_{3} 1}^{H}\right)\right) \\
& +2 \lambda^{*}\left(Z_{t_{2} 3}^{H}\left(\Lambda_{3} Z_{t_{3} 1}^{H}+\Lambda_{2} Z_{t_{3} 2}^{H}\right)+Z_{t_{2} 1}^{H}\left(\Lambda_{3} Z_{t_{3} 3}^{H}-2 v_{s} \lambda Z_{t_{3} 1}^{H}+v_{s} \kappa Z_{t_{3} 2}^{H}\right)\right. \\
& \left.\left.\left.+Z_{t_{2} 2}^{H}\left(-2 v_{s} \lambda Z_{t_{3} 2}^{H}+\Lambda_{2} Z_{t_{3} 3}^{H}+v_{s} \kappa Z_{t_{3} 1}^{H}\right)\right)\right)\right) \\
& \Gamma_{h_{t_{1}} A_{t_{2}}^{0} A_{t_{3}}^{0}}=\frac{i}{4}\left(-Z_{t_{2} 1}^{A}\left(-4 v_{s} \operatorname{Re}\{\lambda \kappa\} Z_{t_{3} 3}^{A} Z_{t_{1} 2}^{H}+\sqrt{2} 2 \operatorname{Re}\left\{T_{\lambda}\right\} Z_{t_{3} 3}^{A} Z_{t_{1} 2}^{H}\right.\right. \\
& +4 v_{s} \operatorname{Re}\{\lambda \kappa\} Z_{t_{3} 2}^{A} Z_{t_{1} 3}^{H}+\sqrt{2} 2 \operatorname{Re}\left\{T_{\lambda}\right\} Z_{t_{3} 2}^{A} Z_{t_{1} 3}^{H}-4 v_{u} \operatorname{Re}\{\lambda \kappa\} Z_{t_{3} 3}^{A} Z_{t_{1} 3}^{H} \\
& \left.+Z_{t_{3} 1}^{A}\left(4 v_{s}|\lambda|^{2} Z_{t_{1} 3}^{H}+g_{+}^{2} v_{d} Z_{t_{1} 1}^{H}-v_{u} \tilde{\lambda} Z_{t_{1} 2}^{H}\right)\right) \\
& +Z_{t_{2} 2}^{A}\left(2 v_{s} \kappa \lambda^{*} Z_{t_{3} 3}^{A} Z_{t_{1} 1}^{H}-\sqrt{2} T_{\lambda}^{*} Z_{t_{3} 3}^{A} Z_{t_{1} 1}^{H}-\sqrt{2} T_{\lambda} Z_{t_{3} 3}^{A} Z_{t_{1} 1}^{H}-2 v_{s} \kappa \lambda^{*} Z_{t_{3} 1}^{A} Z_{t_{1} 3}^{H}\right. \\
& -\sqrt{2} T_{\lambda}^{*} Z_{t_{3} 1}^{A} Z_{t_{1} 3}^{H}-\sqrt{2} T_{\lambda} Z_{t_{3} 1}^{A} Z_{t_{1} 3}^{H}+2 v_{d} \kappa \lambda^{*} Z_{t_{3} 3}^{A} Z_{t_{1} 3}^{H} \\
& +Z_{t_{3} 2}^{A}\left(-4 v_{s}|\lambda|^{2} Z_{t_{1} 3}^{H}-g_{+}^{2} v_{u} Z_{t_{1} 2}^{H}+v_{d} \tilde{\lambda} Z_{t_{1} 1}^{H}\right) \\
& \left.+2 \lambda \kappa^{*}\left(-v_{s} Z_{t_{3} 1}^{A} Z_{t_{1} 3}^{H}+Z_{t_{3} 3}^{A}\left(v_{d} Z_{t_{1} 3}^{H}+v_{s} Z_{t_{1} 1}^{H}\right)\right)\right) \\
& +Z_{t_{2} 3}^{A}\left(-\sqrt{2}\left(-2 \operatorname{Re}\left\{T_{\kappa}\right\} Z_{t_{3} 3}^{A} Z_{t_{1} 3}^{H}+T_{\lambda}^{*}\left(Z_{t_{3} 1}^{A} Z_{t_{1} 2}^{H}+Z_{t_{3} 2}^{A} Z_{t_{1} 1}^{H}\right)\right.\right. \\
& \left.+T_{\lambda}\left(Z_{t_{3} 1}^{A} Z_{t_{1} 2}^{H}+Z_{t_{3} 2}^{A} Z_{t_{1} 1}^{H}\right)\right)+2 \lambda^{*}\left(-Z_{t_{3} 3}^{A}\left(\left(2 v_{d} \lambda+v_{u} \kappa\right) Z_{t_{1} 1}^{H}\right.\right. \\
& \left.\left.+\left(2 v_{u} \lambda+v_{d} \kappa\right) Z_{t_{1} 2}^{H}\right)+\kappa Z_{t_{3} 2}^{A}\left(v_{d} Z_{t_{1} 3}^{H}+v_{s} Z_{t_{1} 1}^{H}\right)+\kappa Z_{t_{3} 1}^{A}\left(v_{s} Z_{t_{1} 2}^{H}+v_{u} Z_{t_{1} 3}^{H}\right)\right) \\
& +2 \kappa^{*}\left(\lambda Z_{t_{3} 2}^{A}\left(v_{d} Z_{t_{1} 3}^{H}+v_{s} Z_{t_{1} 1}^{H}\right)+\lambda Z_{t_{3} 1}^{A}\left(v_{s} Z_{t_{1} 2}^{H}+v_{u} Z_{t_{1} 3}^{H}\right)\right. \\
& \left.\left.\left.-Z_{t_{3} 3}^{A}\left(4 v_{s} \kappa Z_{t_{1} 3}^{H}+v_{d} \lambda Z_{t_{1} 2}^{H}+v_{u} \lambda Z_{t_{1} 1}^{H}\right)\right)\right)\right) \\
& \Gamma_{h_{t_{1}} H_{t_{2}}^{+} H_{t_{3}}^{-}}=\frac{i}{4}\left(-Z_{t_{3} 1}^{+, *}\left(Z_{t_{1} 2}^{H}\left(g_{-}^{2} v_{u} Z_{t_{2} 1}^{+}+v_{d} \bar{\lambda} Z_{t_{2} 2}^{+}\right)+Z_{t_{1} 1}^{H}\left(g_{+}^{2} v_{d} Z_{t_{2} 1}^{+}+v_{u} \bar{\lambda} Z_{t_{2} 2}^{+}\right)\right.\right. \\
& \left.+2 Z_{t_{1} 3}^{H}\left(2 v_{s}|\lambda|^{2} Z_{t_{2} 1}^{+}+\left(2 v_{s} \lambda \kappa^{*}+\sqrt{2} T_{\lambda}\right) Z_{t_{2} 2}^{+}\right)\right) \\
& -Z_{t_{3} 2}^{+, *}\left(Z_{t_{1} 1}^{H}\left(g_{-}^{2} v_{d} Z_{t_{2} 2}^{+}+v_{u} \bar{\lambda} Z_{t_{2} 1}^{+}\right)+Z_{t_{1} 2}^{H}\left(g_{+}^{2} v_{u} Z_{t_{2} 2}^{+}+v_{d} \bar{\lambda} Z_{t_{2} 1}^{+}\right)\right. \\
& \left.\left.+2 Z_{t_{1} 3}^{H}\left(2 v_{s} \lambda^{*}\left(\kappa Z_{t_{2} 1}^{+}+\lambda Z_{t_{2} 2}^{+}\right)+\sqrt{2} T_{\lambda}^{*} Z_{t_{2} 1}^{+}\right)\right)\right) \\
& \Gamma_{A_{t_{1}}^{0} H_{t_{2}}^{+} H_{t_{3}}^{-}}=\frac{1}{4}\left(Z_{t_{3} 2}^{+, *}\left(2\left(2 v_{s} \kappa \lambda^{*}-\sqrt{2} T_{\lambda}^{*}\right) Z_{t_{1} 3}^{A}+v_{d} \bar{\lambda} Z_{t_{1} 2}^{A}+v_{u} \bar{\lambda} Z_{t_{1} 1}^{A}\right) Z_{t_{2} 1}^{+}\right. \\
& \left.-Z_{t_{3} 1}^{+, *}\left(2\left(2 v_{s} \lambda \kappa^{*}-\sqrt{2} T_{\lambda}\right) Z_{t_{1} 3}^{A}+v_{d} \bar{\lambda} Z_{t_{1} 2}^{A}+v_{u} \bar{\lambda} Z_{t_{1} 1}^{A}\right) Z_{t_{2} 2}^{+}\right)
\end{aligned}
$$




\section{One-loop tadpoles}

In this and the subsequent appendices, particles that are denoted with a hat, e.g. $\hat{h}_{i}$, are the unrotated external states. In the corresponding vertices the associated mixing matrix has to be replaced by the identity matrix. Moreover, we have summed her and in the subsequent section in all the vertices implicitly over the colour indices of quarks and squarks.

At the one-loop level, the expressions for the tadpoles of eq. (2.13) are given by

$$
\begin{aligned}
\delta t_{i}= & \frac{3}{2} A_{0}\left(m_{Z}^{2}\right) \Gamma_{\hat{h}_{i}, Z, Z}+3 A_{0}\left(m_{W}^{2}\right) \Gamma_{\hat{h}_{i}, W^{+}, W^{-}}-\sum_{s_{1}=1}^{2} A_{0}\left(m_{H_{s_{1}}^{+}}^{2}\right) \Gamma_{\hat{h}_{i}, H_{s_{1}}^{+}, H_{s_{1}}^{-}} \\
& +4 \sum_{s_{1}=1}^{2} A_{0}\left(m_{\tilde{\chi}_{s_{1}}^{+}}^{2}\right) \Gamma_{\hat{h}_{i}, \tilde{\chi}_{s_{1}}^{+}, \tilde{\chi}_{s_{1}}^{-}} m_{\tilde{\chi}_{s_{1}}^{+}}^{2}-\frac{1}{2} \sum_{s_{1}=1}^{3} A_{0}\left(m_{A_{s_{1}}^{0}}^{2}\right) \Gamma_{\hat{h}_{i}, A_{s_{1}}^{0}, A_{s_{1}}^{0}} \\
& -\frac{1}{2} \sum_{s_{1}=1}^{3} A_{0}\left(m_{h_{s_{1}}}^{2}\right) \Gamma_{\hat{h}_{i}, h_{s_{1}}, h_{s_{1}}}+12 \sum_{s_{1}=1}^{3} A_{0}\left(m_{d_{s_{1}}}^{2}\right) \Gamma_{\hat{h}_{i}, \bar{d}_{s_{1}}, d_{s_{1}}} m_{d_{s_{1}}}^{2} \\
& +4 \sum_{s_{1}=1}^{3} A_{0}\left(m_{e_{s_{1}}}^{2}\right) \Gamma_{\hat{h}_{i}, \bar{e}_{s_{1}}, e_{s_{1}}} m_{e_{s_{1}}}^{2}+12 \sum_{s_{1}=1}^{3} A_{0}\left(m_{u_{s_{1}}}^{2}\right) \Gamma_{\hat{h}_{i}, \bar{u}_{s_{1}}, u_{s_{1}}} m_{u_{s_{1}}}^{2} \\
& +2 \sum_{s_{1}=1}^{5} A_{0}\left(m_{\tilde{\chi}_{s_{1}}^{0}}^{2}\right) \Gamma_{\hat{h}_{i}, \tilde{\chi}_{s_{1}}^{0}, \tilde{\chi}_{s_{1}}^{0}} m_{\tilde{\chi}_{s_{1}}^{0}}^{2}-3 \sum_{s_{1}=1}^{6} A_{0}\left(m_{\tilde{d}_{s_{1}}}^{2}\right) \Gamma_{\hat{h}_{i}, \tilde{d}_{s_{1}}^{*}, \tilde{s}_{s_{1}}} \\
& -3 \sum_{s_{1}=1}^{6} A_{0}\left(m_{\tilde{u}_{s_{1}}}^{2}\right) \Gamma_{\hat{h}_{i}, \tilde{u}_{s_{1}}^{*}, \tilde{u}_{s_{1}}}-\sum_{s_{1}=1}^{3} A_{0}\left(m_{\tilde{\nu}_{s_{1}}}^{2}\right) \Gamma_{\hat{h}_{i}, \tilde{\nu}_{s_{1}}^{*}, \tilde{\nu}_{s_{1}}} \\
& -\sum_{s_{1}=1}^{6} A_{0}\left(m_{\tilde{e}_{s_{1}}}^{2}\right) \Gamma_{\hat{h}_{i}, \tilde{e}_{s_{1}}^{*}, \tilde{e}_{s_{1}}}
\end{aligned}
$$

\section{E One-loop self-energies}

The definitions of the scalar one-loop functions and their explicit analytic expressions can be found in ref. [34].

\section{E.1 Self energy of $Z$-boson}

In agreement with ref. [33] we obtain for the transverse self-energy of the $Z$-boson

$$
\begin{aligned}
\Pi_{Z Z}^{T}\left(p^{2}\right)= & \frac{1}{2} g_{2}^{2} c_{\Theta}^{2}\left(-8 B_{22}\left(p^{2}, m_{W}^{2}, m_{W}^{2}\right)-B_{0}\left(p^{2}, m_{W}^{2}, m_{W}^{2}\right)\left(2 m_{W}^{2}+4 p^{2}\right)\right) \\
& -4 \sum_{s_{1}=1}^{2} \sum_{s_{2}=1}^{2}\left|\Gamma_{Z, H_{s_{1}}^{+}, H_{s_{2}}^{-}}\right|^{2} B_{22}\left(p^{2}, m_{H_{s_{1}}^{+}}^{2}, m_{H_{s_{2}}^{+}}^{2}\right) \\
& +\frac{1}{2} \sum_{s_{1}=1}^{2} \sum_{s_{2}=1}^{2}\left[\left(\left|\Gamma_{Z, \tilde{\chi}_{s_{1}}^{+}, \tilde{\chi}_{s_{2}}^{-}}\right|^{2}+\left|\Gamma_{Z, \tilde{\chi}_{s_{1}}^{+}, \tilde{x}_{s_{2}}^{-}}\right|^{2}\right) H_{0}\left(p^{2}, m_{\tilde{\chi}_{s_{1}}^{+}}^{2}, m_{\tilde{\chi}_{s_{2}}^{+}}^{2}\right)\right. \\
& \left.+4 B_{0}\left(p^{2}, m_{\tilde{\chi}_{s_{1}}^{+}}^{2}, m_{\tilde{\chi}_{s_{2}}^{+}}^{2}\right) m_{\tilde{\chi}_{s_{1}}^{+}} m_{\tilde{\chi}_{s_{2}}^{-}} \operatorname{Re}\left\{\Gamma_{Z, \tilde{\chi}_{s_{1}}^{+}, \tilde{\chi}_{s_{2}}^{-}}^{L *} \Gamma_{Z, \tilde{\chi}_{s_{1}}^{+}, \tilde{\chi}_{s_{2}}^{-}}^{R}\right\}\right]
\end{aligned}
$$




$$
\begin{aligned}
& -4 \sum_{s_{1}=1}^{3} \sum_{s_{2}=1}^{3}\left|\Gamma_{Z, A_{s_{1}}^{0}, h_{s_{2}}}\right|^{2} B_{22}\left(p^{2}, m_{h_{s_{1}}}^{2}, m_{A_{s_{2}}^{0}}^{2}\right) \\
& -4 \sum_{s_{1}=1}^{3} \sum_{s_{2}=1}^{3}\left|\Gamma_{Z, \tilde{\nu}_{s_{1}}^{*}, \tilde{\nu}_{s_{2}}}\right|^{2} B_{22}\left(p^{2}, m_{\tilde{\nu}_{s_{1}}}^{2}, m_{\tilde{\nu}_{s_{2}}}^{2}\right) \\
& +\frac{3}{2} \sum_{s_{1}=1}^{3} \sum_{s_{2}=1}^{3}\left[\left(\left|\Gamma_{Z, \bar{d}_{s_{1}}, d_{s_{2}}}^{L}\right|^{2}+\left|\Gamma_{Z, \bar{d}_{s_{1}}, d_{s_{2}}}^{R}\right|^{2}\right) H_{0}\left(p^{2}, m_{d_{s_{1}}}^{2}, m_{d_{s_{2}}}^{2}\right)\right. \\
& \left.+4 B_{0}\left(p^{2}, m_{d_{s_{1}}}^{2}, m_{d_{s_{2}}}^{2}\right) m_{\bar{d}_{s_{1}}} m_{d_{s_{2}}} \operatorname{Re}\left\{\Gamma_{Z, \bar{d}_{s_{1}}, d_{s_{2}}}^{L *} \Gamma_{Z, \bar{d}_{s_{1}}, d_{s_{2}}}^{R}\right\}\right] \\
& +\frac{1}{2} \sum_{s_{1}=1}^{3} \sum_{s_{2}=1}^{3}\left[\left(\left|\Gamma_{Z, \bar{e}_{s_{1}}, e_{s_{2}}}^{L}\right|^{2}+\left|\Gamma_{Z, \bar{e}_{s_{1}}, e_{s_{2}}}^{R}\right|^{2}\right) H_{0}\left(p^{2}, m_{e_{s_{1}}}^{2}, m_{e_{s_{2}}}^{2}\right)\right. \\
& \left.+4 B_{0}\left(p^{2}, m_{e_{s_{1}}}^{2}, m_{e_{s_{2}}}^{2}\right) m_{\bar{e}_{s_{1}}} m_{e_{s_{2}}} \operatorname{Re}\left\{\Gamma_{Z, \bar{s}_{s_{1}}, e_{s_{2}}}^{L *} \Gamma_{Z, \bar{e}_{s_{1}}, e_{s_{2}}}^{R}\right\}\right] \\
& +\frac{3}{2} \sum_{s_{1}=1}^{3} \sum_{s_{2}=1}^{3}\left[\left(\left|\Gamma_{Z, \bar{u}_{s_{1}}, u_{s_{2}}}^{L}\right|^{2}+\left|\Gamma_{Z, \bar{u}_{s_{1}}, u_{s_{2}}}^{R}\right|^{2}\right) H_{0}\left(p^{2}, m_{u_{s_{1}}}^{2}, m_{u_{s_{2}}}^{2}\right)\right. \\
& \left.+4 B_{0}\left(p^{2}, m_{u_{s_{1}}}^{2}, m_{u_{s_{2}}}^{2}\right) m_{\bar{u}_{s_{1}}} m_{u_{s_{2}}} \operatorname{Re}\left\{\Gamma_{Z, \bar{u}_{s_{1}}, u_{s_{2}}}^{L *} \Gamma_{Z, \bar{u}_{s_{1}}, u_{s_{2}}}^{R}\right\}\right] \\
& +\frac{1}{2} \sum_{s_{1}=1}^{3} \sum_{s_{2}=1}^{3}\left[\left(\left|\Gamma_{Z, \bar{\nu}_{s_{1}}, \nu_{s_{2}}}^{L}\right|^{2}+\left|\Gamma_{Z, \bar{\nu}_{s_{1}}, \nu_{s_{2}}}^{R}\right|^{2}\right) H_{0}\left(p^{2}, 0,0\right)\right. \\
& +\frac{1}{4} \sum_{s_{1}=1}^{5} \sum_{s_{2}=1}^{5}\left[\left(\left|\Gamma_{Z, \tilde{\chi}_{s_{1}}^{0}, \tilde{\chi}_{s_{2}}^{0}}^{L}\right|^{2}+\left|\Gamma_{Z, \tilde{\chi}_{s_{1}}^{0}, \tilde{\chi}_{s_{2}}^{0}}^{R}\right|^{2}\right) H_{0}\left(p^{2}, m_{\tilde{\chi}_{s_{1}}^{0}}^{2}, m_{\tilde{\chi}_{s_{2}}^{0}}^{2}\right)\right. \\
& \left.+4 B_{0}\left(p^{2}, m_{\tilde{\chi}_{s_{1}}^{0}}^{2}, m_{\tilde{\chi}_{s_{2}}^{0}}^{2}\right) m_{\tilde{\chi}_{s_{1}}^{0}} m_{\tilde{\chi}_{s_{2}}^{0}} \operatorname{Re}\left\{\Gamma_{Z, \tilde{\chi}_{s_{1}}^{0}, \tilde{\chi}_{s_{2}}^{0}}^{L *} \Gamma_{Z, \tilde{\chi}_{s_{1}}^{0}, \tilde{\chi}_{s_{2}}^{0}}^{R}\right\}\right] \\
& -12 \sum_{s_{1}=1}^{6} \sum_{s_{2}=1}^{6}\left|\Gamma_{Z, \tilde{d}_{s_{1}}, \tilde{d}_{s_{2}}}\right|^{2} B_{22}\left(p^{2}, m_{\tilde{d}_{s_{1}}}^{2}, m_{\tilde{d}_{s_{2}}}^{2}\right) \\
& -4 \sum_{s_{1}=1}^{6} \sum_{s_{2}=1}^{6}\left|\Gamma_{Z, \tilde{e}_{s_{1}}^{*}, \tilde{e}_{s_{2}}}\right|^{2} B_{22}\left(p^{2}, m_{\tilde{e}_{s_{1}}}^{2}, m_{\tilde{e}_{s_{2}}}^{2}\right) \\
& -12 \sum_{s_{1}=1}^{6} \sum_{s_{2}=1}^{6}\left|\Gamma_{Z, \tilde{u}_{s_{1}}^{*}, \tilde{u}_{s_{2}}}\right|^{2} B_{22}\left(p^{2}, m_{\tilde{u}_{s_{1}}}^{2}, m_{\tilde{u}_{s_{2}}}^{2}\right) \\
& +\frac{1}{2} \sum_{s_{2}=1}^{3}\left|\Gamma_{Z, Z, h_{s_{2}}}\right|^{2} B_{0}\left(p^{2}, m_{Z}^{2}, m_{h_{s_{2}}}^{2}\right)
\end{aligned}
$$

\section{E.2 Self-energy of CP-even Higgs-bosons}

$$
\begin{aligned}
\Pi_{h_{i}, h_{j}}\left(p^{2}\right)= & \frac{7}{4} B_{0}\left(p^{2}, m_{Z}^{2}, m_{Z}^{2}\right) \Gamma_{\hat{h}_{j}, Z, Z}^{*} \Gamma_{\hat{h}_{i}, Z, Z} \\
& +\frac{7}{2} B_{0}\left(p^{2}, m_{W}^{2}, m_{W}^{2}\right) \Gamma_{\hat{h}_{j}, W^{+}, W^{-}}^{*} \Gamma_{\hat{h}_{i}, W^{+}, W^{-}}+2 A_{0}\left(m_{Z}^{2}\right) \Gamma_{\hat{h}_{i}, \hat{h}_{j}, Z, Z} \\
& +4 A_{0}\left(m_{W}^{2}\right) \Gamma_{\hat{h}_{i}, \hat{h}_{j}, W^{+}, W^{-}}-\sum_{s_{1}=1}^{2} A_{0}\left(m_{H_{s_{1}}^{+}}^{2}\right) \Gamma_{\hat{h}_{i}, \hat{h}_{j}, H_{s_{1}}^{+}, H_{s_{1}}^{-}}
\end{aligned}
$$




$$
\begin{aligned}
& +\sum_{s_{1}=1}^{2} \sum_{s_{2}=1}^{2} B_{0}\left(p^{2}, m_{H_{s_{1}}^{+}}^{2}, m_{H_{s_{2}}^{+}}^{2}\right) \Gamma_{\hat{h}_{j}, H_{s_{1}}^{+}, H_{s_{2}}^{-}}^{*} \Gamma_{\hat{h}_{i}, H_{s_{1}}^{+}, H_{s_{2}}^{-}} \\
& -2 \sum_{s_{1}=1}^{2} m_{\tilde{\chi}_{s_{1}}^{+}} \sum_{s_{2}=1}^{2}\left[B _ { 0 } ( p ^ { 2 } , m _ { \tilde { \chi } _ { s _ { 1 } } ^ { + } } ^ { 2 } , m _ { \tilde { \chi } _ { s _ { 2 } } ^ { + } } ^ { 2 } ) m _ { \tilde { \chi } _ { s _ { 2 } } ^ { - } } \left(\Gamma_{\hat{h}_{j}, \tilde{\chi}_{s_{1}}^{+}, \tilde{\chi}_{s_{2}}^{-}}^{L *} \Gamma_{\hat{h}_{i}, \tilde{\chi}_{s_{1}}^{+}, \tilde{\chi}_{s_{2}}^{-}}^{R}\right.\right. \\
& \left.\left.+\Gamma_{\hat{h}_{j}, \tilde{\chi}_{s_{1}}^{+}, \tilde{\chi}_{s_{2}}^{-}}^{R *} \Gamma_{\hat{h}_{i}, \tilde{\chi}_{s_{1}}^{+}, \tilde{\chi}_{s_{2}}^{-}}^{L}\right)\right] \\
& +\sum_{s_{1}=1}^{2} \sum_{s_{2}=1}^{2}\left[G _ { 0 } ( p ^ { 2 } , m _ { \tilde { \chi } _ { s _ { 1 } } ^ { + } } ^ { 2 } , m _ { \tilde { \chi } _ { s _ { 2 } } ^ { + } } ^ { 2 } ) \left(\Gamma_{\hat{h}_{j}, \tilde{\chi}_{s_{1}}^{+}, \tilde{\chi}_{s_{2}}^{-}}^{L *} \Gamma_{\hat{h}_{i}, \tilde{\chi}_{s_{1}}^{+}, \tilde{\chi}_{s_{2}}^{-}}^{L}\right.\right. \\
& \left.\left.+\Gamma_{\hat{h}_{j}, \tilde{\chi}_{s_{1}}^{+}, \tilde{\chi}_{s_{2}}^{-}}^{R *} \Gamma_{\hat{h}_{i}, \tilde{\chi}_{s_{1}}^{+}, \tilde{\chi}_{s_{2}}^{-}}^{R}\right)\right] \\
& -\frac{1}{2} \sum_{s_{1}=1}^{3} A_{0}\left(m_{A_{s_{1}}^{0}}^{2}\right) \Gamma_{\hat{h}_{i}, \hat{h}_{j}, A_{s_{1}}^{0}, A_{s_{1}}^{0}}-\sum_{s_{1}=1}^{3} A_{0}\left(m_{\tilde{\nu}_{s_{1}}}^{2}\right) \Gamma_{\hat{h}_{i}, \hat{h}_{j}, \tilde{\nu}_{s_{1}}^{*}, \tilde{\nu}_{s_{1}}} \\
& -\frac{1}{2} \sum_{s_{1}=1}^{3} A_{0}\left(m_{h_{s_{1}}}^{2}\right) \Gamma_{\hat{h}_{i}, \hat{h}_{j}, h_{s_{1}}, h_{s_{1}}} \\
& +\frac{1}{2} \sum_{s_{1}=1}^{3} \sum_{s_{2}=1}^{3} B_{0}\left(p^{2}, m_{A_{s_{1}}^{0}}^{2}, m_{A_{s_{2}}^{0}}^{2}\right) \Gamma_{\hat{h}_{j}, A_{s_{1}}^{0}, A_{s_{2}}^{0}}^{*} \Gamma_{\hat{h}_{i}, A_{s_{1}}^{0}, A_{s_{2}}^{0}} \\
& +\sum_{s_{1}=1}^{3} \sum_{s_{2}=1}^{3} B_{0}\left(p^{2}, m_{A_{s_{1}}^{0}}^{2}, m_{h_{s_{2}}}^{2}\right) \Gamma_{\hat{h}_{j}, A_{s_{1}}^{0}, h_{s_{2}}}^{*} \Gamma_{\hat{h}_{i}, A_{s_{1}}^{0}, h_{s_{2}}} \\
& +\sum_{s_{1}=1}^{3} \sum_{s_{2}=1}^{3} B_{0}\left(p^{2}, m_{\tilde{\nu}_{s_{1}}}^{2}, m_{\tilde{\nu}_{s_{2}}}^{2}\right) \Gamma_{\hat{h}_{j}, \tilde{\nu}_{s_{1}}^{*}, \tilde{\nu}_{s_{2}}}^{*} \Gamma_{\hat{h}_{i}, \tilde{\nu}_{s_{1}}^{*}, \tilde{\nu}_{s_{2}}} \\
& +\frac{1}{2} \sum_{s_{1}=1}^{3} \sum_{s_{2}=1}^{3} B_{0}\left(p^{2}, m_{h_{s_{1}}}^{2}, m_{h_{s_{2}}}^{2}\right) \Gamma_{\hat{h}_{j}, h_{s_{1}}, h_{s_{2}}}^{*} \Gamma_{\hat{h}_{i}, h_{s_{1}}, h_{s_{2}}} \\
& -6 \sum_{s_{1}=1}^{3} m_{\bar{d}_{s_{1}}} \sum_{s_{2}=1}^{3}\left[B _ { 0 } ( p ^ { 2 } , m _ { d _ { s _ { 1 } } } ^ { 2 } , m _ { d _ { s _ { 2 } } } ^ { 2 } ) m _ { d _ { s _ { 2 } } } \left(\Gamma_{\hat{h}_{j}, \bar{d}_{s_{1}}, d_{s_{2}}}^{L *} \Gamma_{\hat{h}_{i}, \bar{d}_{s_{1}}, d_{s_{2}}}^{R}\right.\right. \\
& \left.\left.+\Gamma_{\hat{h}_{j}, \bar{d}_{s_{1}}, d_{s_{2}}}^{R *} \Gamma_{\hat{h}_{i}, \bar{d}_{s_{1}}, d_{s_{2}}}^{L}\right)\right] \\
& +3 \sum_{s_{1}=1}^{3} \sum_{s_{2}=1}^{3}\left[G _ { 0 } ( p ^ { 2 } , m _ { d _ { s _ { 1 } } } ^ { 2 } , m _ { d _ { s _ { 2 } } } ^ { 2 } ) \left(\Gamma_{\hat{h}_{j}, \bar{d}_{s_{1}}, d_{s_{2}}}^{L *} \Gamma_{\hat{h}_{i}, \bar{d}_{s_{1}}, d_{s_{2}}}^{L}\right.\right. \\
& \left.\left.+\Gamma_{\hat{h}_{j}, \bar{d}_{s_{1}}, d_{s_{2}}}^{R *} \Gamma_{\hat{h}_{i}, \bar{d}_{s_{1}}, d_{s_{2}}}^{R}\right)\right] \\
& -2 \sum_{s_{1}=1}^{3} m_{\bar{e}_{s_{1}}} \sum_{s_{2}=1}^{3}\left[B _ { 0 } ( p ^ { 2 } , m _ { e _ { s _ { 1 } } } ^ { 2 } , m _ { e _ { s _ { 2 } } } ^ { 2 } ) m _ { e _ { s _ { 2 } } } \left(\Gamma_{\hat{h}_{j}, \bar{e}_{s_{1}}, e_{s_{2}}}^{L *} \Gamma_{\hat{h}_{i}, \bar{e}_{s_{1}}, e_{s_{2}}}^{R}\right.\right. \\
& \left.\left.+\Gamma_{\hat{h}_{j}, \bar{e}_{s_{1}}, e_{s_{2}}}^{R *} \Gamma_{\hat{h}_{i}, \bar{e}_{s_{1}}, e_{s_{2}}}^{L}\right)\right] \\
& +\sum_{s_{1}=1}^{3} \sum_{s_{2}=1}^{3}\left[G _ { 0 } ( p ^ { 2 } , m _ { e _ { s _ { 1 } } } ^ { 2 } , m _ { e _ { s _ { 2 } } } ^ { 2 } ) \left(\Gamma_{\hat{h}_{j}, \bar{e}_{s_{1}}, e_{s_{2}}}^{L *} \Gamma_{\hat{h}_{i}, \bar{e}_{s_{1}}, e_{s_{2}}}^{L}\right.\right.
\end{aligned}
$$




$$
\begin{aligned}
& \left.\left.+\Gamma_{\hat{h}_{j}, \bar{e}_{s_{1}}, e_{s_{2}}}^{R *} \Gamma_{\hat{h}_{i}, \bar{e}_{s_{1}}, e_{s_{2}}}^{R}\right)\right] \\
& -6 \sum_{s_{1}=1}^{3} m_{\bar{u}_{s_{1}}} \sum_{s_{2}=1}^{3}\left[B _ { 0 } ( p ^ { 2 } , m _ { u _ { s _ { 1 } } } ^ { 2 } , m _ { u _ { s _ { 2 } } } ^ { 2 } ) m _ { u _ { s _ { 2 } } } \left(\Gamma_{\hat{h}_{j}, \bar{u}_{s_{1}}, u_{s_{2}}}^{L *} \Gamma_{\hat{h}_{i}, \bar{u}_{s_{1}}, u_{s_{2}}}^{R}\right.\right. \\
& \left.\left.+\Gamma_{\hat{h}_{j}, \bar{u}_{s_{1}}, u_{s_{2}}}^{R *} \Gamma_{\hat{h}_{i}, \bar{u}_{s_{1}}, u_{s_{2}}}^{L}\right)\right] \\
& +3 \sum_{s_{1}=1}^{3} \sum_{s_{2}=1}^{3}\left[G _ { 0 } ( p ^ { 2 } , m _ { u _ { s _ { 1 } } } ^ { 2 } , m _ { u _ { s _ { 2 } } } ^ { 2 } ) \left(\Gamma_{\hat{h}_{j}, \bar{u}_{s_{1}}, u_{s_{2}}}^{L *} \Gamma_{\hat{h}_{i}, \bar{u}_{s_{1}}, u_{s_{2}}}^{L}\right.\right. \\
& \left.\left.+\Gamma_{\hat{h}_{j}, \bar{u}_{s_{1}}, u_{s_{2}}}^{R *} \Gamma_{\hat{h}_{i}, \bar{u}_{s_{1}}, u_{s_{2}}}^{R}\right)\right] \\
& -\sum_{s_{1}=1}^{5} m_{\tilde{\chi}_{s_{1}}^{0}} \sum_{s_{2}=1}^{5}\left[B _ { 0 } ( p ^ { 2 } , m _ { \tilde { \chi } _ { s _ { 1 } } ^ { 0 } } ^ { 2 } , m _ { \tilde { \chi } _ { s _ { 2 } } ^ { 0 } } ^ { 2 } ) m _ { \tilde { \chi } _ { s _ { 2 } } ^ { 0 } } \left(\Gamma_{\hat{h}_{j}, \tilde{\chi}_{s_{1}}^{0}, \tilde{\chi}_{s_{2}}^{0}} \Gamma_{\hat{h}_{i}, \tilde{\chi}_{s_{1}}^{0}, \tilde{\chi}_{s_{2}}^{0}}^{R}\right.\right. \\
& \left.\left.+\Gamma_{\hat{h}_{j}, \tilde{\chi}_{s_{1}}^{0}, \tilde{\chi}_{s_{2}}^{0}}^{R *} \Gamma_{\hat{h}_{i}, \tilde{\chi}_{s_{1}}^{0}, \tilde{\chi}_{s_{2}}^{0}}^{L}\right)\right] \\
& +\frac{1}{2} \sum_{s_{1}=1}^{5} \sum_{s_{2}=1}^{5}\left[G _ { 0 } ( p ^ { 2 } , m _ { \tilde { \chi } _ { s _ { 1 } } ^ { 0 } } ^ { 2 } , m _ { \tilde { \chi } _ { s _ { 2 } } ^ { 0 } } ^ { 2 } ) \left(\Gamma_{\hat{h}_{j}, \tilde{\chi}_{s_{1}}^{0}, \tilde{\chi}_{s_{2}}^{0}}^{L} \Gamma_{\hat{h}_{i}, \tilde{\chi}_{s_{1}}^{0}, \tilde{\chi}_{s_{2}}^{0}}^{L}\right.\right. \\
& \left.\left.+\Gamma_{\hat{h}_{j}, \tilde{\chi}_{s_{1}}^{0}, \tilde{\chi}_{s_{2}}^{0}}^{\Gamma_{\hat{h}_{i}}, \tilde{\chi}_{s_{1}}^{0}, \tilde{\chi}_{s_{2}}^{0}}\right)\right] \\
& -3 \sum_{s_{1}=1}^{6} A_{0}\left(m_{\tilde{d}_{s_{1}}}^{2}\right) \Gamma_{\hat{h}_{i}, \hat{h}_{j}, \tilde{d}_{s_{1}}^{*}, \tilde{d}_{s_{1}}}-\sum_{s_{1}=1}^{6} A_{0}\left(m_{\tilde{e}_{s_{1}}}^{2}\right) \Gamma_{\hat{h}_{i}, \hat{h}_{j}, \tilde{e}_{s_{1}}^{*}, \tilde{e}_{s_{1}}} \\
& -3 \sum_{s_{1}=1}^{6} A_{0}\left(m_{\tilde{u}_{s_{1}}}^{2}\right) \Gamma_{\hat{h}_{i}, \hat{h}_{j}, \tilde{u}_{s_{1}}^{*}, \tilde{u}_{s_{1}}} \\
& +3 \sum_{s_{1}=1}^{6} \sum_{s_{2}=1}^{6} B_{0}\left(p^{2}, m_{\tilde{d}_{s_{1}}}^{2}, m_{\tilde{d}_{s_{2}}}^{2}\right) \Gamma_{\hat{h}_{j}, \tilde{d}_{s_{1}}^{*}, \tilde{d}_{s_{2}}}^{*} \Gamma_{\hat{h}_{i}, \tilde{d}_{s_{1}}^{*}, \tilde{d}_{s_{2}}} \\
& +\sum_{s_{1}=1}^{6} \sum_{s_{2}=1}^{6} B_{0}\left(p^{2}, m_{\tilde{e}_{s_{1}}}^{2}, m_{\tilde{e}_{s_{2}}}^{2}\right) \Gamma_{\hat{h}_{j}, \tilde{e}_{s_{1}}^{*}, \tilde{e}_{s_{2}}}^{*} \Gamma_{\hat{h}_{i}, \tilde{e}_{s_{1}}^{*}, \tilde{e}_{s_{2}}} \\
& +3 \sum_{s_{1}=1}^{6} \sum_{s_{2}=1}^{6} B_{0}\left(p^{2}, m_{\tilde{u}_{s_{1}}}^{2}, m_{\tilde{u}_{s_{2}}}^{2}\right) \Gamma_{\hat{h}_{j}, \tilde{u}_{s_{1}}^{*}, \tilde{u}_{s_{2}}}^{*} \Gamma_{\hat{h}_{i}, \tilde{u}_{s_{1}}^{*}, \tilde{u}_{s_{2}}} \\
& +2 \sum_{s_{2}=1}^{2} \Gamma_{\hat{h}_{j}, W^{+}, H_{s_{2}}^{-}}^{*} \Gamma_{\hat{h}_{i}, W^{+}, H_{s_{2}}^{-}} F_{0}\left(p^{2}, m_{H_{s_{2}}^{+}}^{2}, m_{W}^{2}\right) \\
& +\sum_{s_{2}=1}^{3} \Gamma_{\hat{h}_{j}, Z, A_{s_{2}}^{0}}^{*} \Gamma_{\hat{h}_{i}, Z, A_{s_{2}}^{0}} F_{0}\left(p^{2}, m_{A_{s_{2}}^{0}}^{2}, m_{Z}^{2}\right)
\end{aligned}
$$

\section{E.3 Self-energy of CP-odd Higgs-bosons}

$$
\Pi_{A_{i}^{0}, A_{j}^{0}}\left(p^{2}\right)=2 A_{0}\left(m_{Z}^{2}\right) \Gamma_{\hat{A}_{h, i}, \hat{A}_{h, j}, Z, Z}+4 A_{0}\left(m_{W}^{2}\right) \Gamma_{\hat{A}_{h, i}, \hat{A}_{h, j}, W^{+}, W^{-}}
$$




$$
\begin{aligned}
& -\sum_{s_{1}=1}^{2} A_{0}\left(m_{H_{s_{1}}^{+}}^{2}\right) \Gamma_{\hat{A}_{h, i}, \hat{A}_{h, j}, H_{s_{1}}^{+}, H_{s_{1}}^{-}} \\
& +\sum_{s_{1}=1}^{2} \sum_{s_{2}=1}^{2} B_{0}\left(p^{2}, m_{H_{s_{1}}^{+}}^{2}, m_{H_{s_{2}}^{+}}^{2}\right) \Gamma_{\hat{A}_{h, j}, H_{s_{1}}^{+}, H_{s_{2}}^{-}}^{*} \Gamma_{\hat{A}_{h, i}, H_{s_{1}}^{+}, H_{s_{2}}^{-}} \\
& -2 \sum_{s_{1}=1}^{2} m_{\tilde{\chi}_{s_{1}}^{+}} \sum_{s_{2}=1}^{2}\left[B _ { 0 } ( p ^ { 2 } , m _ { \tilde { \chi } _ { s _ { 1 } } ^ { + } } ^ { 2 } , m _ { \tilde { \chi } _ { s _ { 2 } } ^ { + } } ^ { 2 } ) m _ { \tilde { \chi } _ { s _ { 2 } } ^ { - } } \left(\Gamma_{\hat{A}_{h, j}, \tilde{\chi}_{s_{1}}^{+}, \tilde{\chi}_{s_{2}}^{-}}^{\Gamma_{\hat{A}_{h, i},}^{R} \tilde{\chi}_{s_{1}}^{+}, \tilde{\chi}_{s_{2}}^{-}}\right.\right. \\
& \left.\left.+\Gamma_{\hat{A}_{h, j}, \tilde{\chi}_{s_{1}}^{+}, \tilde{\chi}_{s_{2}}^{-}}^{R *} \Gamma_{\hat{A}_{h, i}, \tilde{\chi}_{s_{1}}^{+}, \tilde{\chi}_{s_{2}}^{-}}^{L}\right)\right] \\
& +\sum_{s_{1}=1}^{2} \sum_{s_{2}=1}^{2}\left[G _ { 0 } ( p ^ { 2 } , m _ { \tilde { \chi } _ { s _ { 1 } } ^ { + } } ^ { 2 } , m _ { \tilde { \chi } _ { s _ { 2 } } ^ { + } } ^ { 2 } ) \left(\Gamma_{\hat{A}_{h, j}, \tilde{\chi}_{s_{1}}^{+}, \tilde{\chi}_{s_{2}}^{-}}^{L *} \Gamma_{\hat{A}_{h, i}, \tilde{\chi}_{s_{1}}^{+}, \tilde{\chi}_{s_{2}}^{-}}^{-}\right.\right. \\
& \left.\left.+\Gamma_{\hat{A}_{h, j}, \tilde{\chi}_{s_{1}}^{+}, \tilde{\chi}_{s_{2}}^{-}}^{R *} \Gamma_{\hat{A}_{h, i}, \tilde{\chi}_{s_{1}}^{+}, \tilde{\chi}_{s_{2}}^{-}}^{R}\right)\right] \\
& -\frac{1}{2} \sum_{s_{1}=1}^{3} A_{0}\left(m_{A_{s_{1}}^{0}}^{2}\right) \Gamma_{\hat{A}_{h, i}, \hat{A}_{h, j}, A_{s_{1}}^{0}, A_{s_{1}}^{0}}-\sum_{s_{1}=1}^{3} A_{0}\left(m_{\tilde{\nu}_{s_{1}}}^{2}\right) \Gamma_{\hat{A}_{h, i}, \hat{A}_{h, j}, \tilde{\nu}_{s_{1}}^{*}, \tilde{\nu}_{s_{1}}} \\
& -\frac{1}{2} \sum_{s_{1}=1}^{3} A_{0}\left(m_{h_{s_{1}}}^{2}\right) \Gamma_{\hat{A}_{h, i}, \hat{A}_{h, j}, h_{s_{1}}, h_{s_{1}}} \\
& +\frac{1}{2} \sum_{s_{1}=1}^{3} \sum_{s_{2}=1}^{3} B_{0}\left(p^{2}, m_{A_{s_{1}}^{0}}^{2}, m_{A_{s_{2}}^{0}}^{2}\right) \Gamma_{\hat{A}_{h, j}, A_{s_{1}}^{0}, A_{s_{2}}^{0}}^{*} \Gamma_{\hat{A}_{h, i}, A_{s_{1}}^{0}, A_{s_{2}}^{0}} \\
& +\sum_{s_{1}=1}^{3} \sum_{s_{2}=1}^{3} B_{0}\left(p^{2}, m_{A_{s_{1}}^{0}}^{2}, m_{h_{s_{2}}}^{2}\right) \Gamma_{\hat{A}_{h, j}, A_{s_{1}}^{0}, h_{s_{2}}}^{*} \Gamma_{\hat{A}_{h, i}, A_{s_{1}}^{0}, h_{s_{2}}} \\
& +\frac{1}{2} \sum_{s_{1}=1}^{3} \sum_{s_{2}=1}^{3} B_{0}\left(p^{2}, m_{h_{s_{1}}}^{2}, m_{h_{s_{2}}}^{2}\right) \Gamma_{\hat{A}_{h, j}, h_{s_{1}}, h_{s_{2}}}^{*} \Gamma_{\hat{A}_{h, i}, h_{s_{1}}, h_{s_{2}}} \\
& -6 \sum_{s_{1}=1}^{3} m_{\bar{d}_{s_{1}}} \sum_{s_{2}=1}^{3}\left[B _ { 0 } ( p ^ { 2 } , m _ { d _ { s _ { 1 } } } ^ { 2 } , m _ { d _ { s _ { 2 } } } ^ { 2 } ) m _ { d _ { s _ { 2 } } } \left(\Gamma_{\hat{A}_{h, j}, \bar{d}_{s_{1}}, d_{s_{2}}}^{L *} \Gamma_{\hat{A}_{h, i}, \bar{d}_{s_{1}}, d_{s_{2}}}^{R}\right.\right. \\
& \left.\left.+\Gamma_{\hat{A}_{h, j}, \bar{d}_{s_{1}}, d_{s_{2}}}^{R *} \Gamma_{\hat{A}_{h, i}, \bar{d}_{s_{1}}, d_{s_{2}}}^{L}\right)\right] \\
& +3 \sum_{s_{1}=1}^{3} \sum_{s_{2}=1}^{3}\left[G _ { 0 } ( p ^ { 2 } , m _ { d _ { s _ { 1 } } } ^ { 2 } , m _ { d _ { s _ { 2 } } } ^ { 2 } ) \left(\Gamma_{\hat{A}_{h, j}, \bar{d}_{s_{1}}, d_{s_{2}}}^{L *} \Gamma_{\hat{A}_{h, i}, \bar{d}_{s_{1}}, d_{s_{2}}}^{L}\right.\right. \\
& \left.\left.+\Gamma_{\hat{A}_{h, j}, \bar{d}_{s_{1}}, d_{s_{2}}}^{R *} \Gamma_{\hat{A}_{h, i}, \bar{d}_{s_{1}}, d_{s_{2}}}^{R}\right)\right] \\
& -2 \sum_{s_{1}=1}^{3} m_{\bar{e}_{s_{1}}} \sum_{s_{2}=1}^{3}\left[B _ { 0 } ( p ^ { 2 } , m _ { e _ { s _ { 1 } } } ^ { 2 } , m _ { e _ { s _ { 2 } } } ^ { 2 } ) m _ { e _ { s _ { 2 } } } \left(\Gamma_{\hat{A}_{h, j}, \bar{e}_{s_{1}}, e_{s_{2}}}^{L *} \Gamma_{\hat{A}_{h, i}, \bar{e}_{s_{1}}, e_{s_{2}}}^{R}\right.\right. \\
& \left.\left.+\Gamma_{\hat{A}_{h, j}, \bar{e}_{s_{1}}, e_{s_{2}}}^{R *} \Gamma_{\hat{A}_{h, i}, \bar{e}_{s_{1}}, e_{s_{2}}}^{L}\right)\right] \\
& +\sum_{s_{1}=1}^{3} \sum_{s_{2}=1}^{3}\left[G _ { 0 } ( p ^ { 2 } , m _ { e _ { s _ { 1 } } } ^ { 2 } , m _ { e _ { s _ { 2 } } } ^ { 2 } ) \left(\Gamma_{\hat{A}_{h, j}, \bar{e}_{s_{1}}, e_{s_{2}}}^{L *} \Gamma_{\hat{A}_{h, i}, \bar{e}_{s_{1}}, e_{s_{2}}}^{L}\right.\right.
\end{aligned}
$$




$$
\begin{aligned}
& \left.\left.+\Gamma_{\hat{A}_{h, j}, \bar{e}_{s_{1}}, e_{s_{2}}}^{R *} \Gamma_{\hat{A}_{h, i}, \bar{e}_{s_{1}}, e_{s_{2}}}^{R}\right)\right] \\
& -6 \sum_{s_{1}=1}^{3} m_{\bar{u}_{s_{1}}} \sum_{s_{2}=1}^{3}\left[B _ { 0 } ( p ^ { 2 } , m _ { u _ { s _ { 1 } } } ^ { 2 } , m _ { u _ { s _ { 2 } } } ^ { 2 } ) m _ { u _ { s _ { 2 } } } \left(\Gamma_{\hat{A}_{h, j}, \bar{u}_{s_{1}}, u_{s_{2}}}^{L *} \Gamma_{\hat{A}_{h, i}, \bar{u}_{s_{1}}, u_{s_{2}}}^{R}\right.\right. \\
& \left.\left.+\Gamma_{\hat{A}_{h, j}, \bar{u}_{s_{1}}, u_{s_{2}}}^{R *} \Gamma_{\hat{A}_{h, i}, \bar{u}_{s_{1}}, u_{s_{2}}}^{L}\right)\right] \\
& +3 \sum_{s_{1}=1}^{3} \sum_{s_{2}=1}^{3}\left[G _ { 0 } ( p ^ { 2 } , m _ { u _ { s _ { 1 } } } ^ { 2 } , m _ { u _ { s _ { 2 } } } ^ { 2 } ) \left(\Gamma_{\hat{A}_{h, j}, \bar{u}_{s_{1}}, u_{s_{2}}}^{L *} \Gamma_{\hat{A}_{h, i}, \bar{u}_{s_{1}}, u_{s_{2}}}^{L}\right.\right. \\
& \left.\left.+\Gamma_{\hat{A}_{h, j}, \bar{u}_{s_{1}}, u_{s_{2}}}^{R *} \Gamma_{\hat{A}_{h, i}, \bar{u}_{s_{1}}, u_{s_{2}}}^{R}\right)\right] \\
& -\sum_{s_{1}=1}^{5} m_{\tilde{\chi}_{s_{1}}^{0}} \sum_{s_{2}=1}^{5}\left[B _ { 0 } ( p ^ { 2 } , m _ { \tilde { \chi } _ { s _ { 1 } } ^ { 0 } } ^ { 2 } , m _ { \tilde { \chi } _ { s _ { 2 } } ^ { 0 } } ^ { 2 } ) m _ { \tilde { \chi } _ { s _ { 2 } } ^ { 0 } } \left(\Gamma_{\hat{A}_{h, j}, \tilde{\chi}_{s_{1}}^{0}, \tilde{\chi}_{s_{2}}^{0}}^{L *} \Gamma_{\hat{A}_{h, i}, \tilde{\chi}_{s_{1}}^{0}, \tilde{\chi}_{s_{2}}^{0}}^{R}\right.\right. \\
& \left.\left.+\Gamma_{\hat{A}_{h, j}, \tilde{\chi}_{s_{1}}^{0}, \tilde{\chi}_{s_{2}}^{0}}^{R *} \Gamma_{\hat{A}_{h, i}, \tilde{\chi}_{s_{1}}^{0}, \tilde{\chi}_{s_{2}}^{0}}^{L}\right)\right] \\
& +\frac{1}{2} \sum_{s_{1}=1}^{5} \sum_{s_{2}=1}^{5}\left[G _ { 0 } ( p ^ { 2 } , m _ { \tilde { \chi } _ { s _ { 1 } } ^ { 0 } } ^ { 2 } , m _ { \tilde { \chi } _ { s _ { 2 } } ^ { 0 } } ^ { 2 } ) \left(\Gamma_{\hat{A}_{h, j}, \tilde{\chi}_{s_{1}}^{0}, \tilde{\chi}_{s_{2}}^{0}}^{L *} \Gamma_{\hat{A}_{h, i}, \tilde{\chi}_{s_{1}}^{0}, \tilde{\chi}_{s_{2}}^{0}}^{L}\right.\right. \\
& \left.\left.+\Gamma_{\hat{A}_{h, j}, \tilde{\chi}_{s_{1}}^{0}, \tilde{\chi}_{s_{2}}^{0}}^{R *} \Gamma_{\hat{A}_{h, i}, \tilde{\chi}_{s_{1}}^{0}, \tilde{\chi}_{s_{2}}^{0}}^{R}\right)\right] \\
& -3 \sum_{s_{1}=1}^{6} A_{0}\left(m_{\tilde{d}_{s_{1}}}^{2}\right) \Gamma_{\hat{A}_{h, i}, \hat{A}_{h, j}, \tilde{d}_{s_{1}}^{*}, \tilde{d}_{s_{1}}}-\sum_{s_{1}=1}^{6} A_{0}\left(m_{\tilde{e}_{s_{1}}}^{2}\right) \Gamma_{\hat{A}_{h, i}, \hat{A}_{h, j}, \tilde{e}_{s_{1}}^{*}, \tilde{e}_{s_{1}}} \\
& -3 \sum_{s_{1}=1}^{6} A_{0}\left(m_{\tilde{u}_{s_{1}}}^{2}\right) \Gamma_{\hat{A}_{h, i}, \hat{A}_{h, j}, \tilde{u}_{s_{1}}^{*}, \tilde{u}_{s_{1}}} \\
& +3 \sum_{s_{1}=1}^{6} \sum_{s_{2}=1}^{6} B_{0}\left(p^{2}, m_{\tilde{d}_{s_{1}}}^{2}, m_{\tilde{d}_{s_{2}}}^{2}\right) \Gamma_{\hat{A}_{h, j}, \tilde{d}_{s_{1}}^{*}, \tilde{d}_{s_{2}}}^{*} \Gamma_{\hat{A}_{h, i}, \tilde{d}_{s_{1}}^{*}, \tilde{d}_{s_{2}}} \\
& +\sum_{s_{1}=1}^{6} \sum_{s_{2}=1}^{6} B_{0}\left(p^{2}, m_{\tilde{e}_{s_{1}}}^{2}, m_{\tilde{e}_{s_{2}}}^{2}\right) \Gamma_{\hat{A}_{h, j}, \tilde{e}_{s_{1}}^{*}, \tilde{e}_{s_{2}}}^{*} \Gamma_{\hat{A}_{h, i}, \tilde{e}_{s_{1}}^{*}, \tilde{e}_{s_{2}}} \\
& +3 \sum_{s_{1}=1}^{6} \sum_{s_{2}=1}^{6} B_{0}\left(p^{2}, m_{\tilde{u}_{s_{1}}}^{2}, m_{\tilde{u}_{s_{2}}}^{2}\right) \Gamma_{\hat{A}_{h, j}, \tilde{u}_{s_{1}}^{*}, \tilde{u}_{s_{2}}}^{*} \Gamma_{\hat{A}_{h, i}, \tilde{u}_{s_{1}}^{*}, \tilde{u}_{s_{2}}} \\
& +2 \sum_{s_{2}=1}^{2} \Gamma_{\hat{A}_{h, j}, W^{+}, H_{s_{2}}^{-}}^{*} \Gamma_{\hat{A}_{h, i}, W^{+}, H_{s_{2}}^{-}} F_{0}\left(p^{2}, m_{H_{s_{2}}^{+}}^{2}, m_{W}^{2}\right) \\
& +\sum_{s_{2}=1}^{3} \Gamma_{\hat{A}_{h, j}, Z, h_{s_{2}}}^{*} \Gamma_{\hat{A}_{h, i}, Z, h_{s_{2}}} F_{0}\left(p^{2}, m_{h_{s_{2}}}^{2}, m_{Z}^{2}\right)
\end{aligned}
$$

\section{E.4 Self-energy of the charged Higgs-boson}

$$
\begin{aligned}
\Pi_{H_{i}^{-}, H_{j}^{-}}\left(p^{2}\right)= & \frac{7}{2} B_{0}\left(p^{2}, m_{Z}^{2}, m_{W}^{2}\right) \Gamma_{\hat{H}_{j}^{+}, W^{-}, Z^{2}}^{*} \Gamma_{\hat{H}_{i}^{+}, W^{-}, Z}+2 A_{0}\left(m_{Z}^{2}\right) \Gamma_{\hat{H}_{i}^{+}, \hat{H}_{j}^{-}, Z, Z} \\
& +4 A_{0}\left(m_{W}^{2}\right) \Gamma_{\hat{H}_{i}^{+}, \hat{H}_{j}^{-}, W^{+}, W^{-}}-\sum_{s_{1}=1}^{2} A_{0}\left(m_{H_{s_{1}}^{+}}^{2}\right) \Gamma_{\hat{H}_{i}^{+}, \hat{H}_{j}^{-}, H_{s_{1}}^{+}, H_{s_{1}}^{-}}
\end{aligned}
$$




$$
\begin{aligned}
& -2 \sum_{s_{1}=1}^{2} m_{\tilde{\chi}_{s_{1}}^{-}} \sum_{s_{2}=1}^{5}\left[B _ { 0 } ( p ^ { 2 } , m _ { \tilde { \chi } _ { s _ { 1 } } ^ { + } } ^ { 2 } , m _ { \tilde { \chi } _ { s _ { 2 } } ^ { 0 } } ^ { 2 } ) m _ { \tilde { \chi } _ { s _ { 2 } } ^ { 0 } } \left(\Gamma_{\hat{H}_{j}^{+}, \tilde{\chi}_{s_{1}}^{-}, \tilde{\chi}_{s_{2}}^{0}}^{L *} \Gamma_{\hat{H}_{i}^{+}, \tilde{\chi}_{s_{1}}^{-}, \tilde{\chi}_{s_{2}}^{0}}^{R}\right.\right. \\
& \left.\left.+\Gamma_{\hat{H}_{j}^{+}, \tilde{\chi}_{s_{1}}^{-}, \tilde{\chi}_{s_{2}}^{0}}^{R *} \Gamma_{\hat{H}_{i}^{+}, \tilde{\chi}_{s_{1}}^{-}, \tilde{\chi}_{s_{2}}^{0}}^{L}\right)\right] \\
& +\sum_{s_{1}=1}^{2} \sum_{s_{2}=1}^{5}\left[G _ { 0 } ( p ^ { 2 } , m _ { \tilde { \chi } _ { s _ { 1 } } ^ { + } } ^ { 2 } , m _ { \tilde { \chi } _ { s _ { 2 } } ^ { 0 } } ^ { 2 } ) \left(\Gamma_{\hat{H}_{j}^{+}, \tilde{\chi}_{s_{1}}^{-}, \tilde{\chi}_{s_{2}}^{0}}^{L *} \Gamma_{\hat{H}_{i}^{+}, \tilde{\chi}_{s_{1}}^{-}, \tilde{\chi}_{s_{2}}^{0}}^{L}\right.\right. \\
& \left.\left.+\Gamma_{\hat{H}_{j}^{+}, \tilde{\chi}_{s_{1}}^{-}, \tilde{\chi}_{s_{2}}^{0}}^{R *} \Gamma_{\hat{H}_{i}^{+}, \tilde{\chi}_{s_{1}}^{-}, \tilde{\chi}_{s_{2}}^{0}}^{R}\right)\right] \\
& -\frac{1}{2} \sum_{s_{1}=1}^{3} A_{0}\left(m_{A_{s_{1}}^{0}}^{2}\right) \Gamma_{\hat{H}_{i}^{+}, \hat{H}_{j}^{-}, A_{s_{1}}^{0}, A_{s_{1}}^{0}}-\sum_{s_{1}=1}^{3} A_{0}\left(m_{\tilde{\nu}_{s_{1}}}^{2}\right) \Gamma_{\hat{H}_{i}^{+}, \hat{H}_{j}^{-}, \tilde{\nu}_{s_{1}}^{*}, \tilde{\nu}_{s_{1}}} \\
& -\frac{1}{2} \sum_{s_{1}=1}^{3} A_{0}\left(m_{h_{s_{1}}}^{2}\right) \Gamma_{\hat{H}_{i}^{+}, \hat{H}_{j}^{-}, h_{s_{1}}, h_{s_{1}}} \\
& +\sum_{s_{1}=1}^{3} \sum_{s_{2}=1}^{2} B_{0}\left(p^{2}, m_{A_{s_{1}}^{0}}^{2}, m_{H_{s_{2}}^{+}}^{2}\right) \Gamma_{\hat{H}_{j}^{+}, A_{s_{1}}^{0}, H_{s_{2}}^{-}}^{*} \Gamma_{\hat{H}_{i}^{+}, A_{s_{1}}^{0}, H_{s_{2}}^{-}} \\
& +\sum_{s_{1}=1}^{3} \sum_{s_{2}=1}^{2} B_{0}\left(p^{2}, m_{h_{s_{1}}}^{2}, m_{H_{s_{2}}^{+}}^{2}\right) \Gamma_{\hat{H}_{j}^{+}, h_{s_{1}}, H_{s_{2}}^{-}}^{*} \Gamma_{\hat{H}_{i}^{+}, h_{s_{1}}, H_{s_{2}}^{-}} \\
& -6 \sum_{s_{1}=1}^{3} m_{d_{s_{1}}} \sum_{s_{2}=1}^{3}\left[B _ { 0 } ( p ^ { 2 } , m _ { d _ { s _ { 1 } } } ^ { 2 } , m _ { u _ { s _ { 2 } } } ^ { 2 } ) m _ { \overline { u } _ { s _ { 2 } } } \left(\Gamma_{\hat{H}_{j}^{+}, d_{s_{1}}, \bar{u}_{s_{2}}}^{L *} \Gamma_{\hat{H}_{i}^{+}, d_{s_{1}}, \bar{u}_{s_{2}}}^{R}\right.\right. \\
& \left.\left.+\Gamma_{\hat{H}_{j}^{+}, d_{s_{1}}, \bar{u}_{s_{2}}}^{R *} \Gamma_{\hat{H}_{i}^{+}, d_{s_{1}}, \bar{u}_{s_{2}}}^{L}\right)\right] \\
& +3 \sum_{s_{1}=1}^{3} \sum_{s_{2}=1}^{3}\left[G _ { 0 } ( p ^ { 2 } , m _ { d _ { s _ { 1 } } } ^ { 2 } , m _ { u _ { s _ { 2 } } } ^ { 2 } ) \left(\Gamma_{\hat{H}_{j}^{+}, d_{s_{1}}, \bar{u}_{s_{2}}}^{L *} \Gamma_{\hat{H}_{i}^{+}, d_{s_{1}}, \bar{u}_{s_{2}}}^{L}\right.\right. \\
& \left.\left.+\Gamma_{\hat{H}_{j}^{+}, d_{s_{1}}, \bar{u}_{s_{2}}}^{R *} \Gamma_{\hat{H}_{i}^{+}, d_{s_{1}}, \bar{u}_{s_{2}}}^{R}\right)\right] \\
& -2 \sum_{s_{1}=1}^{3} m_{e_{s_{1}}} \sum_{s_{2}=1}^{3}\left[B _ { 0 } ( p ^ { 2 } , m _ { e _ { s _ { 1 } } } ^ { 2 } , m _ { \nu _ { s _ { 2 } } } ^ { 2 } ) m _ { \overline { \nu } _ { s _ { 2 } } } \left(\Gamma_{\hat{H}_{j}^{+}, e_{s_{1}}, \bar{\nu}_{s_{2}}}^{L *} \Gamma_{\hat{H}_{i}^{+}, e_{s_{1}}, \bar{\nu}_{s_{2}}}^{R}\right.\right. \\
& \left.\left.+\Gamma_{\hat{H}_{j}^{+}, e_{s_{1}}, \bar{\nu}_{s_{2}}}^{R *} \Gamma_{\hat{H}_{i}^{+}, e_{s_{1}}, \bar{\nu}_{s_{2}}}^{L}\right)\right] \\
& +\sum_{s_{1}=1}^{3} \sum_{s_{2}=1}^{3}\left[G _ { 0 } ( p ^ { 2 } , m _ { e _ { s _ { 1 } } } ^ { 2 } , m _ { \nu _ { s _ { 2 } } } ^ { 2 } ) \left(\Gamma_{\hat{H}_{j}^{+}, e_{s_{1}}, \bar{\nu}_{s_{2}}}^{L *} \Gamma_{\hat{H}_{i}^{+}, e_{s_{1}}, \bar{\nu}_{s_{2}}}^{L}\right.\right. \\
& \left.\left.+\Gamma_{\hat{H}_{j}^{+}, e_{s_{1}}, \bar{\nu}_{s_{2}}}^{R *} \Gamma_{\hat{H}_{i}^{+}, e_{s_{1}}, \bar{\nu}_{s_{2}}}^{R}\right)\right] \\
& -3 \sum_{s_{1}=1}^{6} A_{0}\left(m_{\tilde{d}_{s_{1}}}^{2}\right) \Gamma_{\hat{H}_{i}^{+}, \hat{H}_{j}^{-}, \tilde{d}_{s_{1}}^{*}, \tilde{d}_{s_{1}}}-\sum_{s_{1}=1}^{6} A_{0}\left(m_{\tilde{e}_{s_{1}}}^{2}\right) \Gamma_{\hat{H}_{i}^{+}, \hat{H}_{j}^{-}, \tilde{e}_{s_{1}}^{*}, \tilde{e}_{s_{1}}} \\
& -3 \sum_{s_{1}=1}^{6} A_{0}\left(m_{\tilde{u}_{s_{1}}}^{2}\right) \Gamma_{\hat{H}_{i}^{+}, \hat{H}_{j}^{-}, \tilde{u}_{s_{1}}^{*}, \tilde{u}_{s_{1}}}
\end{aligned}
$$




$$
\begin{aligned}
& +\sum_{s_{1}=1}^{6} \sum_{s_{2}=1}^{3} B_{0}\left(p^{2}, m_{\tilde{e}_{s_{1}}}^{2}, m_{\tilde{\nu}_{s_{2}}}^{2}\right) \Gamma_{\hat{H}_{j}^{+}, \tilde{e}_{s_{1}}, \tilde{\nu}_{s_{2}}^{*}}^{*} \Gamma_{\hat{H}_{i}^{+}, \tilde{e}_{s_{1}}, \tilde{\nu}_{s_{2}}^{*}}^{6} \\
& +3 \sum_{s_{1}=1}^{6} \sum_{s_{2}=1}^{6} B_{0}\left(p^{2}, m_{\tilde{d}_{s_{1}}}^{2}, m_{\tilde{u}_{s_{2}}}^{2}\right) \Gamma_{\hat{H}_{j}^{+}, \tilde{d}_{s_{1}}, \tilde{u}_{s_{2}}^{*}}^{*} \Gamma_{\hat{H}_{i}^{+}, \tilde{d}_{s_{1}}, \tilde{u}_{s_{2}}^{*}} \\
& +\sum_{s_{2}=1}^{2} \Gamma_{\hat{H}_{j}^{+}, \gamma, H_{s_{2}}^{-}}^{*} \Gamma_{\hat{H}_{i}^{+}, \gamma, H_{s_{2}}^{-}} F_{0}\left(p^{2}, m_{H_{s_{2}}^{+}}^{2}, 0\right) \\
& +\sum_{s_{2}=1}^{2} \Gamma_{\hat{H}_{j}^{+}, Z, H_{s_{2}}^{-}}^{*} \Gamma_{\hat{H}_{i}^{+}, Z, H_{s_{2}}^{-}} F_{0}\left(p^{2}, m_{H_{s_{2}}^{+}}^{2}, m_{Z}^{2}\right) \\
& +\sum_{s_{2}=1}^{3} \Gamma_{\hat{H}_{j}^{+}, W^{-}, A_{s_{2}}^{0}}^{*} \Gamma_{\hat{H}_{i}^{+}, W^{-}, A_{s_{2}}^{0}} F_{0}\left(p^{2}, m_{A_{s_{2}}^{0}}^{2}, m_{W}^{2}\right) \\
& +\sum_{s_{2}=1}^{3} \Gamma_{\hat{H}_{j}^{+}, W^{-}, h_{s_{2}}}^{*} \Gamma_{\hat{H}_{i}^{+}, W^{-}, h_{s_{2}}} F_{0}\left(p^{2}, m_{h_{s_{2}}}^{2}, m_{W}^{2}\right)
\end{aligned}
$$

\section{E.5 Self-energy of neutralinos}

$$
\begin{aligned}
& \sum_{\tilde{\chi}_{i}^{0}, \tilde{\chi}_{j}^{0}}^{S}\left(p^{2}\right)=\sum_{s_{1}=1}^{2} \sum_{s_{2}=1}^{2} B_{0}\left(p^{2}, m_{\tilde{\chi}_{s_{2}}^{+}}^{2}, m_{H_{s_{1}}^{+}}^{2}\right) \Gamma_{\hat{\chi}_{j}^{0}, H_{s_{1}}^{+}, \tilde{\chi}_{s_{2}}^{-}}^{L *} m_{\tilde{\chi}_{s_{2}}^{-}} \Gamma_{\tilde{\chi}_{i}^{0}, H_{s_{1}}^{+}, \tilde{\chi}_{s_{2}}^{-}}^{R} \\
& +\sum_{s_{1}=1}^{3} \sum_{s_{2}=1}^{3} B_{0}\left(p^{2}, 0,0\right) \Gamma_{\tilde{\chi}_{j}^{0}, \tilde{\nu}_{s_{1}}^{*}, \nu_{s_{2}}}^{L *} m_{\nu_{s_{2}}} \Gamma_{\tilde{\chi}_{i}^{0}, \tilde{\nu}_{s_{1}}^{*}, \nu_{s_{2}}}^{R} \\
& +\frac{1}{2} \sum_{s_{1}=1}^{3} \sum_{s_{2}=1}^{5} B_{0}\left(p^{2}, m_{\tilde{\chi}_{s_{2}}^{0}}^{2}, m_{A_{s_{1}}^{0}}^{2}\right) \Gamma_{\tilde{\chi}_{j}^{0}, A_{s_{1}}^{0}, \tilde{\chi}_{s_{2}}^{0}}^{L *} m_{\tilde{\chi}_{s_{2}}^{0}} \Gamma_{\tilde{\chi}_{i}^{0}, A_{s_{1}}^{0}, \tilde{\chi}_{s_{2}}^{0}}^{R} \\
& +\frac{1}{2} \sum_{s_{1}=1}^{3} \sum_{s_{2}=1}^{5} B_{0}\left(p^{2}, m_{\tilde{\chi}_{s_{2}}^{0}}^{2}, m_{h_{s_{1}}}^{2}\right) \Gamma_{\tilde{\chi}_{j}^{0}, h_{s_{1}}, \tilde{\chi}_{s_{2}}^{0}}^{L *} m_{\tilde{\chi}_{s_{2}}^{0}} \Gamma_{\hat{\chi}_{i}^{0}, h_{s_{1}}, \tilde{\chi}_{s_{2}}^{0}}^{R} \\
& +3 \sum_{s_{1}=1}^{6} \sum_{s_{2}=1}^{3} B_{0}\left(p^{2}, m_{d_{s_{2}}}^{2}, m_{\tilde{d}_{s_{1}}}^{2}\right) \Gamma_{\tilde{\chi}_{j}^{0}, \tilde{d}_{s_{1}}^{*}, d_{s_{2}}}^{L *} m_{d_{s_{2}}} \Gamma_{\tilde{\chi}_{i}^{0}, \tilde{d}_{s_{1}}^{*}, d_{s_{2}}}^{R} \\
& +\sum_{s_{1}=1}^{6} \sum_{s_{2}=1}^{3} B_{0}\left(p^{2}, m_{e_{s_{2}}}^{2}, m_{\tilde{e}_{s_{1}}}^{2}\right) \Gamma_{\hat{\chi}_{j}^{0}, \tilde{e}_{s_{1}}^{*}, e_{s_{2}}}^{L *} m_{e_{s_{2}}} \Gamma_{\hat{\chi}_{i}^{0}, \tilde{e}_{s_{1}}^{*}, e_{s_{2}}}^{R} \\
& +3 \sum_{s_{1}=1}^{6} \sum_{s_{2}=1}^{3} B_{0}\left(p^{2}, m_{u_{s_{2}}}^{2}, m_{\tilde{u}_{s_{1}}}^{2}\right) \Gamma_{\tilde{\chi}_{j}^{0}, \tilde{u}_{s_{1}}^{*}, u_{s_{2}}}^{L *} m_{u_{s_{2}}} \Gamma_{\hat{\chi}_{i}^{0}, \tilde{u}_{s_{1}}^{*}, u_{s_{2}}}^{R} \\
& -4 \sum_{s_{2}=1}^{2} B_{0}\left(p^{2}, m_{\tilde{\chi}_{s_{2}}^{+}}^{2}, m_{W}^{2}\right) \Gamma_{\hat{\chi}_{j}^{0}, W^{+}, \tilde{\chi}_{s_{2}}^{-}}^{R *} m_{\tilde{\chi}_{s_{2}}^{-}} \Gamma_{\hat{\chi}_{i}^{0}, W^{+}, \tilde{\chi}_{s_{2}}^{-}}^{L} \\
& -2 \sum_{s_{2}=1}^{5} B_{0}\left(p^{2}, m_{\tilde{\chi}_{s_{2}}^{0}}^{2}, m_{Z}^{2}\right) \Gamma_{\tilde{\chi}_{j}^{0}, Z, \tilde{\chi}_{s_{2}}^{0}}^{R *} m_{\tilde{\chi}_{s_{2}}^{0}} \Gamma_{\tilde{\chi}_{i}^{0}, Z, \tilde{\chi}_{s_{2}}^{0}}^{L} \\
& \Sigma_{\tilde{\chi}_{i}^{0}, \tilde{\chi}_{j}^{0}}^{R}\left(p^{2}\right)=\sum_{s_{1}=1}^{2} \sum_{s_{2}=1}^{2} B_{0}\left(p^{2}, m_{\tilde{\chi}_{s_{2}}^{+}}^{2}, m_{H_{s_{1}}^{+}}^{2}\right) \Gamma_{\tilde{\chi}_{j}^{0}, H_{s_{1}}^{+}, \tilde{\chi}_{s_{2}}^{-}}^{L *} m_{\tilde{\chi}_{s_{2}}^{-}} \Gamma_{\tilde{\hat{\chi}}_{i}^{0}, H_{s_{1}}^{+}, \tilde{\chi}_{s_{2}}^{-}}^{R}
\end{aligned}
$$




$$
\begin{aligned}
& +\sum_{s_{1}=1}^{3} \sum_{s_{2}=1}^{3} B_{0}\left(p^{2}, 0,0\right) \Gamma_{\tilde{\chi}_{j}^{0}, \tilde{\nu}_{s_{1}}^{*}, \nu_{s_{2}}}^{L *} m_{\nu_{s_{2}}} \Gamma_{\tilde{\chi}_{i}^{0}, \tilde{\nu}_{s_{1}}^{*}, \nu_{s_{2}}}^{R} \\
& +\frac{1}{2} \sum_{s_{1}=1}^{3} \sum_{s_{2}=1}^{5} B_{0}\left(p^{2}, m_{\tilde{\chi}_{s_{2}}^{0}}^{2}, m_{A_{s_{1}}^{0}}^{2}\right) \Gamma_{\tilde{\chi}_{j}^{0}, A_{s_{1}}^{0}, \tilde{\chi}_{s_{2}}^{0}}^{L *} m_{\tilde{\chi}_{s_{2}}^{0}} \Gamma_{\tilde{\chi}_{i}^{0}, A_{s_{1}}^{0}, \tilde{\chi}_{s_{2}}^{0}}^{R} \\
& +\frac{1}{2} \sum_{s_{1}=1}^{3} \sum_{s_{2}=1}^{5} B_{0}\left(p^{2}, m_{\tilde{\chi}_{s_{2}}^{0}}^{2}, m_{h_{s_{1}}}^{2}\right) \Gamma_{\tilde{\chi}_{j}^{0}, h_{s_{1}}, \tilde{\chi}_{s_{2}}^{0}}^{L *} m_{\tilde{\chi}_{s_{2}}^{0}} \Gamma_{\tilde{\tilde{\chi}}_{i}^{0}, h_{s_{1}}, \tilde{\chi}_{s_{2}}^{0}}^{R} \\
& +3 \sum_{s_{1}=1}^{6} \sum_{s_{2}=1}^{3} B_{0}\left(p^{2}, m_{d_{s_{2}}}^{2}, m_{\tilde{d}_{s_{1}}}^{2}\right) \Gamma_{\tilde{\chi}_{j}^{0}, \tilde{d}_{1}, d_{s_{2}}}^{L *} m_{d_{s_{2}}} \Gamma_{\hat{\chi}_{i}^{0}, \tilde{d}_{s_{1}}^{*}, d_{s_{2}}}^{R} \\
& +\sum_{s_{1}=1}^{6} \sum_{s_{2}=1}^{3} B_{0}\left(p^{2}, m_{e_{s_{2}}}^{2}, m_{\tilde{e}_{s_{1}}}^{2}\right) \Gamma_{\tilde{\tilde{\chi}}_{j}^{0}, \tilde{e}_{s_{1}}^{*}, e_{s_{2}}}^{L *} m_{e_{s_{2}}} \Gamma_{\tilde{\tilde{\chi}}_{i}^{0}, \tilde{e}_{s_{1}}^{*}, e_{s_{2}}}^{R} \\
& +3 \sum_{s_{1}=1}^{6} \sum_{s_{2}=1}^{3} B_{0}\left(p^{2}, m_{u_{s_{2}}}^{2}, m_{\tilde{u}_{s_{1}}}^{2}\right) \Gamma_{\tilde{\chi}_{j}^{0}, \tilde{u}_{s_{1}}^{*}, u_{s_{2}}}^{L *} m_{u_{s_{2}}} \Gamma_{\tilde{\chi}_{i}^{0}, \tilde{u}_{s_{1}}^{*}, u_{s_{2}}}^{R} \\
& -4 \sum_{s_{2}=1}^{2} B_{0}\left(p^{2}, m_{\tilde{\chi}_{s_{2}}^{+}}^{2}, m_{W}^{2}\right) \Gamma_{\tilde{\chi}_{j}^{0}, W^{+}, \tilde{\chi}_{s_{2}}^{-}}^{R *} m_{\tilde{\chi}_{s_{2}}^{-}} \Gamma_{\hat{\chi}_{i}^{0}, W^{+}, \tilde{\chi}_{s_{2}}^{-}}^{L} \\
& -2 \sum_{s_{2}=1}^{5} B_{0}\left(p^{2}, m_{\tilde{\chi}_{s_{2}}^{0}}^{2}, m_{Z}^{2}\right) \Gamma_{\tilde{\chi}_{j}^{0}, Z, \tilde{\chi}_{s_{2}}^{0}}^{R *} m_{\tilde{\chi}_{s_{2}}^{0}} \Gamma_{\tilde{\chi}_{i}^{0}, Z, \tilde{\chi}_{s_{2}}^{0}}^{L} \\
& \Sigma_{\tilde{\chi}_{i}^{0}, \tilde{\chi}_{j}^{0}}^{L}\left(p^{2}\right)=\sum_{s_{1}=1}^{2} \sum_{s_{2}=1}^{2} B_{0}\left(p^{2}, m_{\tilde{\chi}_{s_{2}}^{+}}^{2}, m_{H_{s_{1}}^{+}}^{2}\right) \Gamma_{\tilde{\chi}_{j}^{0}, H_{s_{1}}^{+}, \tilde{\chi}_{s_{2}}^{-}}^{L *} m_{\tilde{\chi}_{s_{2}}^{-}} \Gamma_{\tilde{\chi}_{i}^{0}, H_{s_{1}}^{+}, \tilde{\chi}_{s_{2}}^{-}}^{R} \\
& +\sum_{s_{1}=1}^{3} \sum_{s_{2}=1}^{3} B_{0}\left(p^{2}, 0,0\right) \Gamma_{\tilde{\chi}_{j}^{0}, \tilde{\nu}_{s_{1}}^{*}, \nu_{s_{2}}}^{L *} m_{\nu_{s_{2}}} \Gamma_{\tilde{\chi}_{i}^{0}, \tilde{\nu}_{s_{1}}^{*}, \nu_{s_{2}}}^{R} \\
& +\frac{1}{2} \sum_{s_{1}=1}^{3} \sum_{s_{2}=1}^{5} B_{0}\left(p^{2}, m_{\tilde{\chi}_{s_{2}}^{0}}^{2}, m_{A_{s_{1}}^{0}}^{2}\right) \Gamma_{\tilde{\chi}_{j}^{0}, A_{s_{1}}^{0}, \tilde{\chi}_{s_{2}}^{0}}^{L *} m_{\tilde{\chi}_{s_{2}}^{0}} \Gamma_{\hat{\chi}_{i}^{0}, A_{s_{1}}^{0}, \tilde{\chi}_{s_{2}}^{0}}^{R} \\
& +\frac{1}{2} \sum_{s_{1}=1}^{3} \sum_{s_{2}=1}^{5} B_{0}\left(p^{2}, m_{\tilde{\chi}_{s_{2}}^{0}}^{2}, m_{h_{s_{1}}}^{2}\right) \Gamma_{\tilde{\chi}_{j}^{0}, h_{s_{1}}, \tilde{\chi}_{s_{2}}^{0}}^{L *} m_{\tilde{\chi}_{s_{2}}^{0}} \Gamma_{\tilde{\chi}_{i}^{0}, h_{s_{1}}, \tilde{\chi}_{s_{2}}^{0}}^{R} \\
& +3 \sum_{s_{1}=1}^{6} \sum_{s_{2}=1}^{3} B_{0}\left(p^{2}, m_{d_{s_{2}}}^{2}, m_{\tilde{d}_{s_{1}}}^{2}\right) \Gamma_{\tilde{\chi}_{j}^{0}, \tilde{s}_{1}, d_{s_{2}}^{*}}^{L *} m_{d_{s_{2}}} \Gamma_{\tilde{\chi}_{i}^{0}, \tilde{d}_{s_{1}}^{*}, d_{s_{2}}}^{R} \\
& +\sum_{s_{1}=1}^{6} \sum_{s_{2}=1}^{3} B_{0}\left(p^{2}, m_{e_{s_{2}}}^{2}, m_{\tilde{e}_{s_{1}}}^{2}\right) \Gamma_{\tilde{\tilde{\chi}}_{j}^{0}}^{L *} \tilde{e}_{s_{1}}^{*}, e_{s_{2}} m_{e_{s_{2}}} \Gamma_{\tilde{\tilde{\chi}}_{i}^{0}, \tilde{e}_{s_{1}}^{*}, e_{s_{2}}}^{R} \\
& +3 \sum_{s_{1}=1}^{6} \sum_{s_{2}=1}^{3} B_{0}\left(p^{2}, m_{u_{s_{2}}}^{2}, m_{\tilde{u}_{s_{1}}}^{2}\right) \Gamma_{\tilde{\chi}_{j}^{0}, \tilde{u}_{s_{1}}^{*}, u_{s_{2}}}^{L *} m_{u_{s_{2}}} \Gamma_{\tilde{\chi}_{i}^{0}, \tilde{u}_{s_{1}}^{*}, u_{s_{2}}}^{R} \\
& -4 \sum_{s_{2}=1}^{2} B_{0}\left(p^{2}, m_{\tilde{\chi}_{s_{2}}^{+}}^{2}, m_{W}^{2}\right) \Gamma_{\hat{\chi}_{j}^{0}, W^{+}, \tilde{\chi}_{s_{2}}^{-}}^{R *} m_{\tilde{\chi}_{s_{2}}^{-}} \Gamma_{\hat{\chi}_{i}^{0}, W^{+}, \tilde{\chi}_{s_{2}}^{-}}^{L}
\end{aligned}
$$




$$
-2 \sum_{s_{2}=1}^{5} B_{0}\left(p^{2}, m_{\tilde{\chi}_{s_{2}}^{0}}^{2}, m_{Z}^{2}\right) \Gamma_{\hat{\chi}_{j}^{0}, Z, \tilde{\chi}_{s_{2}}^{0}}^{R *} m_{\tilde{\chi}_{s_{2}}^{0}} \Gamma_{\hat{\chi}_{i}^{0}, Z, \tilde{\chi}_{s_{2}}^{0}}^{L}
$$

\section{E.6 Self-energy of charginos}

$$
\begin{aligned}
& \sum_{\tilde{\chi}_{i}^{+}, \tilde{\chi}_{j}^{+}}^{S}\left(p^{2}\right)=\sum_{s_{1}=1}^{2} \sum_{s_{2}=1}^{5} B_{0}\left(p^{2}, m_{\tilde{\chi}_{s_{2}}^{0}}^{2}, m_{H_{s_{1}}^{+}}^{2}\right) \Gamma_{\tilde{\chi}_{j}^{+}, H_{s_{1}}^{-}, \tilde{\chi}_{s_{2}}^{0}}^{L *} m_{\tilde{\chi}_{s_{2}}^{0}} \Gamma_{\tilde{\chi}_{i}^{+}, H_{s_{1}}^{-}, \tilde{\chi}_{s_{2}}^{0}}^{R} \\
& +\sum_{s_{1}=1}^{3} \sum_{s_{2}=1}^{2} B_{0}\left(p^{2}, m_{\tilde{\chi}_{s_{2}}^{+}}^{2}, m_{A_{s_{1}}^{0}}^{2}\right) \Gamma_{\tilde{\chi}_{j}^{+}, A_{s_{1}}^{0}, \tilde{\chi}_{s_{2}}^{-}}^{L *} m_{\tilde{\chi}_{s_{2}}^{-}} \Gamma_{\hat{\chi}_{i}^{+}, A_{s_{1}}^{0}, \tilde{\chi}_{s_{2}}^{-}}^{R} \\
& +\sum_{s_{1}=1}^{3} \sum_{s_{2}=1}^{2} B_{0}\left(p^{2}, m_{\tilde{\chi}_{s_{2}}^{+}}^{2}, m_{h_{s_{1}}}^{2}\right) \Gamma_{\tilde{\chi}_{j}^{+}, h_{s_{1}}, \tilde{\chi}_{s_{2}}^{-}}^{L *} m_{\tilde{\chi}_{s_{2}}^{-}} \Gamma_{\tilde{\chi}_{i}^{+}, h_{s_{1}}, \tilde{\chi}_{s_{2}}^{-}}^{R} \\
& +\sum_{s_{1}=1}^{3} \sum_{s_{2}=1}^{3} B_{0}\left(p^{2}, m_{e_{s_{2}}}^{2}, m_{\tilde{\nu}_{s_{1}}}^{2}\right) \Gamma_{\tilde{\chi}_{j}^{+}, \tilde{\nu}_{s_{1}}^{*}, e_{s_{2}}}^{L *} m_{e_{s_{2}}} \Gamma_{\tilde{\chi}_{i}^{+}, \tilde{\nu}_{s_{1}}^{*}, e_{s_{2}}}^{R} \\
& +3 \sum_{s_{1}=1}^{6} \sum_{s_{2}=1}^{3} B_{0}\left(p^{2}, m_{d_{s_{2}}}^{2}, m_{\tilde{u}_{s_{1}}}^{2}\right) \Gamma_{\tilde{\chi}_{j}^{+}, \tilde{u}_{s_{1}}^{*}, d_{s_{2}}}^{L *} m_{d_{s_{2}}} \Gamma_{\tilde{\chi}_{i}^{+}, \tilde{u}_{s_{1}}^{*}, d_{s_{2}}}^{R} \\
& +3 \sum_{s_{1}=1}^{6} \sum_{s_{2}=1}^{3} B_{0}\left(p^{2}, m_{u_{s_{2}}}^{2}, m_{\tilde{d}_{s_{1}}}^{2}\right) \Gamma_{\hat{\chi}_{j}^{+}, \tilde{d}_{s_{1}}, \bar{u}_{s_{2}}}^{L *} m_{\bar{u}_{s_{2}}} \Gamma_{\hat{\chi}_{i}^{+}, \tilde{d}_{s_{1}}, \bar{u}_{s_{2}}}^{R} \\
& +\sum_{s_{1}=1}^{6} \sum_{s_{2}=1}^{3} B_{0}\left(p^{2}, m_{\nu_{s_{2}}}^{2}, m_{\tilde{e}_{s_{1}}}^{2}\right) \Gamma_{\hat{\chi}_{j}^{+}, \tilde{e}_{s_{1}}, \bar{\nu}_{s_{2}}}^{L *} m_{\bar{\nu}_{s_{2}}} \Gamma_{\hat{\chi}_{i}^{+}, \tilde{e}_{s_{1}}, \bar{\nu}_{s_{2}}}^{R} \\
& -4 \sum_{s_{2}=1}^{2} B_{0}\left(p^{2}, m_{\tilde{\chi}_{s_{2}}^{+}}^{2}, 0\right) \Gamma_{\hat{\chi}_{j}^{+}, \gamma, \tilde{\chi}_{s_{2}}^{-}}^{R *} m_{\tilde{\chi}_{s_{2}}^{-}} \Gamma_{\hat{\chi}_{i}^{+}, \gamma, \tilde{\chi}_{s_{2}}^{-}}^{L} \\
& -4 \sum_{s_{2}=1}^{2} B_{0}\left(p^{2}, m_{\tilde{\chi}_{s_{2}}^{+}}^{2}, m_{Z}^{2}\right) \Gamma_{\hat{\chi}_{j}^{+}, Z, \tilde{\chi}_{s_{2}}^{-}}^{R *} m_{\tilde{\chi}_{s_{2}}^{-}} \Gamma_{\hat{\chi}_{i}^{+}, Z, \tilde{\chi}_{s_{2}}^{-}}^{L} \\
& -4 \sum_{s_{2}=1}^{5} B_{0}\left(p^{2}, m_{\tilde{\chi}_{s_{2}}^{0}}^{2}, m_{W}^{2}\right) \Gamma_{\tilde{\chi}_{j}^{+}, W^{-}, \tilde{\chi}_{s_{2}}^{0}}^{R *} m_{\tilde{\chi}_{s_{2}}^{0}} \Gamma_{\hat{\chi}_{i}^{+}, W^{-}, \tilde{\chi}_{s_{2}}^{0}}^{L} \\
& \Sigma_{\tilde{\chi}_{i}^{+}, \tilde{\chi}_{j}^{+}}^{R}\left(p^{2}\right)=\sum_{s_{1}=1}^{2} \sum_{s_{2}=1}^{5} B_{0}\left(p^{2}, m_{\tilde{\chi}_{s_{2}}^{0}}^{2}, m_{H_{s_{1}}^{+}}^{2}\right) \Gamma_{\hat{\chi}_{j}^{+}, H_{s_{1}}^{-}, \tilde{\chi}_{s_{2}}^{0}}^{L *} m_{\tilde{\chi}_{s_{2}}^{0}} \Gamma_{\tilde{\chi}_{i}^{+}, H_{s_{1}}^{-}, \tilde{\chi}_{s_{2}}^{0}}^{R} \\
& +\sum_{s_{1}=1}^{3} \sum_{s_{2}=1}^{2} B_{0}\left(p^{2}, m_{\tilde{\chi}_{s_{2}}^{+}}^{2}, m_{A_{s_{1}}^{0}}^{2}\right) \Gamma_{\tilde{\chi}_{j}^{+}, A_{s_{1}}^{0}, \tilde{\chi}_{s_{2}}^{-}}^{L *} m_{\tilde{\chi}_{s_{2}}^{-}} \Gamma_{\tilde{\chi}_{i}^{+}, A_{s_{1}}^{0}, \tilde{\chi}_{s_{2}}^{-}}^{R} \\
& +\sum_{s_{1}=1}^{3} \sum_{s_{2}=1}^{2} B_{0}\left(p^{2}, m_{\tilde{\chi}_{s_{2}}^{+}}^{2}, m_{h_{s_{1}}}^{2}\right) \Gamma_{\hat{\chi}_{j}^{+}, h_{s_{1}}, \tilde{\chi}_{s_{2}}^{-}}^{L *} m_{\tilde{\chi}_{s_{2}}^{-}} \Gamma_{\hat{\chi}_{i}^{+}, h_{s_{1}}, \tilde{\chi}_{s_{2}}^{-}}^{R} \\
& +\sum_{s_{1}=1}^{3} \sum_{s_{2}=1}^{3} B_{0}\left(p^{2}, m_{e_{s_{2}}}^{2}, m_{\tilde{\nu}_{s_{1}}}^{2}\right) \Gamma_{\tilde{\chi}_{j}^{+}, \tilde{\nu}_{s_{1}}^{*}, e_{s_{2}}}^{L *} m_{e_{s_{2}}} \Gamma_{\tilde{\chi}_{i}^{+}, \tilde{\nu}_{s_{1}}^{*}, e_{s_{2}}}^{R} \\
& +3 \sum_{s_{1}=1}^{6} \sum_{s_{2}=1}^{3} B_{0}\left(p^{2}, m_{d_{s_{2}}}^{2}, m_{\tilde{u}_{s_{1}}}^{2}\right) \Gamma_{\tilde{\chi}_{j}^{+}, \tilde{u}_{s_{1}}^{*}, d_{s_{2}}}^{L *} m_{d_{s_{2}}} \Gamma_{\tilde{\chi}_{i}^{+}, \tilde{u}_{s_{1}}^{*}, d_{s_{2}}}^{R}
\end{aligned}
$$




$$
\begin{aligned}
& +3 \sum_{s_{1}=1}^{6} \sum_{s_{2}=1}^{3} B_{0}\left(p^{2}, m_{u_{s_{2}}}^{2}, m_{\tilde{d}_{s_{1}}}^{2}\right) \Gamma_{\tilde{\chi}_{j}^{+}, \tilde{d}_{s_{1}}, \bar{u}_{s_{2}}}^{L *} m_{\bar{u}_{s_{2}}} \Gamma_{\tilde{\chi}_{i}^{+}, \tilde{d}_{s_{1}}, \bar{u}_{s_{2}}}^{R} \\
& +\sum_{s_{1}=1}^{6} \sum_{s_{2}=1}^{3} B_{0}\left(p^{2}, m_{\nu_{s_{2}}}^{2}, m_{\tilde{e}_{s_{1}}}^{2}\right) \Gamma_{\hat{\tilde{\chi}}_{j}^{+}, \tilde{e}_{s_{1}}, \bar{\nu}_{s_{2}}}^{L *} m_{\bar{\nu}_{s_{2}}} \Gamma_{\hat{\tilde{\chi}}_{i}^{+}, \tilde{e}_{s_{1}}, \bar{\nu}_{s_{2}}}^{R} \\
& -4 \sum_{s_{2}=1}^{2} B_{0}\left(p^{2}, m_{\tilde{\chi}_{s_{2}}^{+}}^{2}, 0\right) \Gamma_{\hat{\chi}_{j}^{+}, \gamma, \tilde{\chi}_{s_{2}}}^{R *} m_{\tilde{\chi}_{s_{2}}^{-}} \Gamma_{\hat{\chi}_{i}^{+}, \gamma, \tilde{\chi}_{s_{2}}^{-}}^{L} \\
& -4 \sum_{s_{2}=1}^{2} B_{0}\left(p^{2}, m_{\tilde{\chi}_{s_{2}}^{+}}^{2}, m_{Z}^{2}\right) \Gamma_{\hat{\chi}_{j}^{+}, Z, \tilde{\chi}_{s_{2}}^{-}}^{R *} m_{\tilde{\chi}_{s_{2}}^{-}} \Gamma_{\hat{\chi}_{i}^{+}, Z, \tilde{\chi}_{s_{2}}^{-}}^{L} \\
& -4 \sum_{s_{2}=1}^{5} B_{0}\left(p^{2}, m_{\tilde{\chi}_{s_{2}}^{0}}^{2}, m_{W}^{2}\right) \Gamma_{\tilde{\hat{\chi}}_{j}^{+}, W^{-}, \tilde{\chi}_{s_{2}}^{0}}^{R *} m_{\tilde{\chi}_{s_{2}}^{0}} \Gamma_{\hat{\chi}_{i}^{+}, W^{-}, \tilde{\chi}_{s_{2}}^{0}}^{L} \\
& \Sigma_{\tilde{\chi}_{i}^{+}, \tilde{\chi}_{j}^{+}}^{L}\left(p^{2}\right)=\sum_{s_{1}=1}^{2} \sum_{s_{2}=1}^{5} B_{0}\left(p^{2}, m_{\tilde{\chi}_{s_{2}}^{0}}^{2}, m_{H_{s_{1}}^{+}}^{2}\right) \Gamma_{\hat{\chi}_{j}^{+}, H_{s_{1}}^{-}, \tilde{\chi}_{s_{2}}^{0}}^{L *} m_{\tilde{\chi}_{s_{2}}^{0}} \Gamma_{\tilde{\chi}_{i}^{+}, H_{s_{1}}^{-}, \tilde{\chi}_{s_{2}}^{0}}^{R} \\
& +\sum_{s_{1}=1}^{3} \sum_{s_{2}=1}^{2} B_{0}\left(p^{2}, m_{\tilde{\chi}_{s_{2}}^{+}}^{2}, m_{A_{s_{1}}^{0}}^{2}\right) \Gamma_{\tilde{\chi}_{j}^{+}, A_{s_{1}}^{0}, \tilde{\chi}_{s_{2}}^{-}}^{L *} m_{\tilde{\chi}_{s_{2}}^{-}} \Gamma_{\tilde{\chi}_{i}^{+}, A_{s_{1}}^{0}, \tilde{\chi}_{s_{2}}^{-}}^{R} \\
& +\sum_{s_{1}=1}^{3} \sum_{s_{2}=1}^{2} B_{0}\left(p^{2}, m_{\tilde{\chi}_{s_{2}}^{+}}^{2}, m_{h_{s_{1}}}^{2}\right) \Gamma_{\hat{\chi}_{j}^{+}, h_{s_{1}}, \tilde{\chi}_{s_{2}}^{-}}^{L *} m_{\tilde{\chi}_{s_{2}}^{-}} \Gamma_{\tilde{\chi}_{i}^{+}, h_{s_{1}}, \tilde{\chi}_{s_{2}}^{-}}^{R} \\
& +\sum_{s_{1}=1}^{3} \sum_{s_{2}=1}^{3} B_{0}\left(p^{2}, m_{e_{s_{2}}}^{2}, m_{\tilde{\nu}_{s_{1}}}^{2}\right) \Gamma_{\tilde{\chi}_{j}^{+}, \tilde{\nu}_{s_{1}}^{*}, e_{s_{2}}}^{L *} m_{e_{s_{2}}} \Gamma_{\tilde{\chi}_{i}^{+}, \tilde{\nu}_{s_{1}}^{*}, e_{s_{2}}}^{R} \\
& +3 \sum_{s_{1}=1}^{6} \sum_{s_{2}=1}^{3} B_{0}\left(p^{2}, m_{d_{s_{2}}}^{2}, m_{\tilde{u}_{s_{1}}}^{2}\right) \Gamma_{\tilde{\chi}_{j}^{+}, \tilde{u}_{s_{1}}^{*}, d_{s_{2}}}^{L *} m_{d_{s_{2}}} \Gamma_{\tilde{\chi}_{i}^{+}, \tilde{u}_{s_{1}}^{*}, d_{s_{2}}}^{R} \\
& +3 \sum_{s_{1}=1}^{6} \sum_{s_{2}=1}^{3} B_{0}\left(p^{2}, m_{u_{s_{2}}}^{2}, m_{\tilde{d}_{s_{1}}}^{2}\right) \Gamma_{\hat{\chi}_{j}^{+}, \tilde{d}_{s_{1}}, \bar{u}_{s_{2}}}^{L *} m_{\bar{u}_{s_{2}}} \Gamma_{\hat{\chi}_{i}^{+}, \tilde{d}_{s_{1}}, \bar{u}_{s_{2}}}^{R} \\
& +\sum_{s_{1}=1}^{6} \sum_{s_{2}=1}^{3} B_{0}\left(p^{2}, m_{\nu_{s_{2}}}^{2}, m_{\tilde{e}_{s_{1}}}^{2}\right) \Gamma_{\hat{\chi}_{j}^{+}, \tilde{e}_{s_{1}}, \bar{\nu}_{s_{2}}}^{L *} m_{\bar{\nu}_{s_{2}}} \Gamma_{\tilde{\chi}_{i}^{+}, \tilde{e}_{s_{1}}, \bar{\nu}_{s_{2}}}^{R} \\
& -4 \sum_{s_{2}=1}^{2} B_{0}\left(p^{2}, m_{\tilde{\chi}_{s_{2}}^{+}}^{2}, 0\right) \Gamma_{\hat{\chi}_{j}^{+}, \gamma, \tilde{\chi}_{s_{2}}}^{R *} m_{\tilde{\chi}_{s_{2}}^{-}} \Gamma_{\hat{\chi}_{i}^{+}, \gamma, \tilde{\chi}_{s_{2}}^{-}}^{L} \\
& -4 \sum_{s_{2}=1}^{2} B_{0}\left(p^{2}, m_{\tilde{\chi}_{s_{2}}^{+}}^{2}, m_{Z}^{2}\right) \Gamma_{\hat{\chi}_{j}^{+}, Z, \tilde{\chi}_{s_{2}}^{-}}^{R *} m_{\tilde{\chi}_{s_{2}}^{-}} \Gamma_{\hat{\chi}_{i}^{+}, Z, \tilde{\chi}_{s_{2}}^{-}}^{L} \\
& -4 \sum_{s_{2}=1}^{5} B_{0}\left(p^{2}, m_{\tilde{\chi}_{s_{2}}^{0}}^{2}, m_{W}^{2}\right) \Gamma_{\hat{\chi}_{j}^{+}, W^{-}, \tilde{\chi}_{s_{2}}^{0}}^{R *} m_{\tilde{\chi}_{s_{2}}^{0}} \Gamma_{\hat{\chi}_{i}^{+}, W^{-}, \tilde{\chi}_{s_{2}}^{0}}^{L}
\end{aligned}
$$

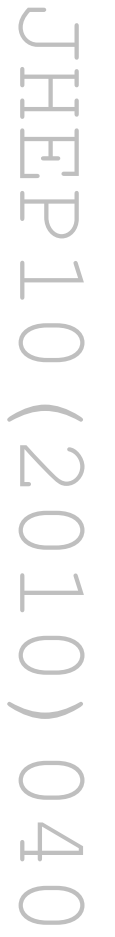

\section{E.7 Self-energy of sleptons}

$$
\Pi_{\tilde{e}_{i}, \tilde{e}_{j}}\left(p^{2}\right)=2 A_{0}\left(m_{Z}^{2}\right) \Gamma_{\hat{\tilde{e}}_{i}^{*}, \hat{e}_{j}, Z, Z}+4 A_{0}\left(m_{W}^{2}\right) \Gamma_{\hat{\tilde{e}}_{i}^{*}, \hat{e}_{j}, W^{+}, W^{-}}
$$




$$
\begin{aligned}
& -\sum_{s_{1}=1}^{2} A_{0}\left(m_{H_{s_{1}}^{+}}^{2}\right) \Gamma_{\hat{\tilde{e}}_{i}^{*}, \hat{\tilde{e}}_{j}, H_{s_{1}}^{+}, H_{s_{1}}^{-}} \\
& +\sum_{s_{1}=1}^{2} \sum_{s_{2}=1}^{3} B_{0}\left(p^{2}, m_{H_{s_{1}}^{+}}^{2}, m_{\tilde{\nu}_{s_{2}}}^{2}\right) \Gamma_{\tilde{e}_{j}^{*}, H_{s_{1}}^{-}, \tilde{\nu}_{s_{2}}}^{*} \Gamma_{\hat{\tilde{e}}_{i}^{*}, H_{s_{1}}^{-}, \tilde{\nu}_{s_{2}}} \\
& -2 \sum_{s_{1}=1}^{2} m_{\tilde{\chi}_{s_{1}}^{-}} \sum_{s_{2}=1}^{3}\left[B _ { 0 } ( p ^ { 2 } , m _ { \tilde { \chi } _ { s _ { 1 } } ^ { + } } ^ { 2 } , 0 ) m _ { \nu _ { s _ { 2 } } } \left(\Gamma_{\tilde{\hat{e}}_{j}^{*}, \tilde{\chi}_{s_{1}}^{-}, \nu_{s_{2}}}^{L *} \Gamma_{\tilde{\hat{e}}_{i}^{*}, \tilde{\chi}_{s_{1}}^{-}, \nu_{s_{2}}}^{R}\right.\right. \\
& \left.\left.+\Gamma_{\tilde{\hat{e}}_{j}^{*}, \tilde{\chi}_{s_{1}}, \nu_{s_{2}}}^{R *} \Gamma_{\hat{e}_{i}^{*}, \tilde{\chi}_{s_{1}}^{-}, \nu_{s_{2}}}^{L}\right)\right] \\
& +\sum_{s_{1}=1}^{2} \sum_{s_{2}=1}^{3}\left[G _ { 0 } ( p ^ { 2 } , m _ { \tilde { \chi } _ { s _ { 1 } } ^ { + } } ^ { 2 } , 0 ) \left(\Gamma_{\hat{e}_{j}^{*}, \tilde{\chi}_{s_{1}}^{-}, \nu_{s_{2}}}^{L *} \Gamma_{\tilde{e}_{i}^{*}, \tilde{\chi}_{s_{1}}^{-}, \nu_{s_{2}}}^{L}\right.\right. \\
& \left.\left.+\Gamma_{\hat{e}_{j}^{*}, \tilde{\chi}_{s_{1}}^{-}, \nu_{s_{2}}}^{R *} \Gamma_{\hat{e}_{i}^{*}, \tilde{\chi}_{s_{1}}^{-}, \nu_{s_{2}}}^{R}\right)\right] \\
& -\frac{1}{2} \sum_{s_{1}=1}^{3} A_{0}\left(m_{A_{s_{1}}^{0}}^{2}\right) \Gamma_{\hat{\tilde{e}}_{i}^{*}, \hat{\tilde{e}}_{j}, A_{s_{1}}^{0}, A_{s_{1}}^{0}}-\sum_{s_{1}=1}^{3} A_{0}\left(m_{\tilde{\nu}_{s_{1}}}^{2}\right) \Gamma_{\hat{\tilde{e}}_{i}^{*}, \hat{\tilde{e}}_{j}, \tilde{\nu}_{s_{1}}^{*}, \tilde{\nu}_{s_{1}}} \\
& -\frac{1}{2} \sum_{s_{1}=1}^{3} A_{0}\left(m_{h_{s_{1}}}^{2}\right) \Gamma_{\hat{\tilde{e}}_{i}^{*}, \hat{\tilde{e}}_{j}, h_{s_{1}}, h_{s_{1}}} \\
& +\sum_{s_{1}=1}^{3} \sum_{s_{2}=1}^{6} B_{0}\left(p^{2}, m_{A_{s_{1}}^{0}}^{2}, m_{\tilde{e}_{s_{2}}}^{2}\right) \Gamma_{\tilde{e}_{j}^{*}, A_{s_{1}}^{0}, \tilde{e}_{s_{2}}}^{*} \Gamma_{\hat{\tilde{e}}_{i}^{*}, A_{s_{1}}^{0}, \tilde{e}_{s_{2}}} \\
& +\sum_{s_{1}=1}^{3} \sum_{s_{2}=1}^{6} B_{0}\left(p^{2}, m_{h_{s_{1}}}^{2}, m_{\tilde{e}_{s_{2}}}^{2}\right) \Gamma_{\hat{e}_{j}^{*}, h_{s_{1}}, \tilde{e}_{s_{2}}}^{*} \Gamma_{\hat{\tilde{e}}_{i}^{*}, h_{s_{1}}, \tilde{e}_{s_{2}}} \\
& -2 \sum_{s_{1}=1}^{5} m_{\tilde{\chi}_{s_{1}}^{0}} \sum_{s_{2}=1}^{3}\left[B _ { 0 } ( p ^ { 2 } , m _ { \tilde { \chi } _ { s _ { 1 } } ^ { 0 } } ^ { 2 } , m _ { e _ { s _ { 2 } } } ^ { 2 } ) m _ { e _ { s _ { 2 } } } \left(\Gamma_{\tilde{\hat{e}}_{j}^{*}, \tilde{\chi}_{s_{1}}^{0}, e_{s_{2}}}^{L *} \Gamma_{\hat{e}_{i}^{*}, \tilde{\chi}_{s_{1}}^{0}, e_{s_{2}}}^{R}\right.\right. \\
& \left.\left.+\Gamma_{\tilde{\hat{e}}_{j}^{*}, \tilde{\chi}_{s_{1}}^{0}, e_{s_{2}}}^{R *} \Gamma_{\tilde{\hat{e}}_{i}^{*}, \tilde{\chi}_{s_{1}}^{0}, e_{s_{2}}}^{L}\right)\right] \\
& +\sum_{s_{1}=1}^{5} \sum_{s_{2}=1}^{3}\left[G _ { 0 } ( p ^ { 2 } , m _ { \tilde { \chi } _ { s _ { 1 } } ^ { 0 } } ^ { 2 } , m _ { e _ { s _ { 2 } } } ^ { 2 } ) \left(\Gamma_{\tilde{\hat{e}}_{j}^{*}, \tilde{\chi}_{s_{1}}^{0}, e_{s_{2}}}^{L *} \Gamma_{\hat{e}_{i}^{*}, \tilde{\chi}_{s_{1}}^{0}, e_{s_{2}}}^{L}\right.\right. \\
& \left.\left.+\Gamma_{\tilde{\hat{e}}_{j}^{*}, \tilde{\chi}_{s_{1}}^{0}, e_{s_{2}}}^{R *} \Gamma_{\tilde{\hat{e}}_{i}^{*}, \tilde{\chi}_{s_{1}}^{0}, e_{s_{2}}}^{R}\right)\right] \\
& -3 \sum_{s_{1}=1}^{6} A_{0}\left(m_{\tilde{d}_{s_{1}}}^{2}\right) \Gamma_{\hat{\tilde{e}}_{i}^{*}, \hat{\tilde{e}}_{j}, \tilde{d}_{s_{1}}^{*}, \tilde{d}_{s_{1}}}-\sum_{s_{1}=1}^{6} A_{0}\left(m_{\tilde{e}_{s_{1}}}^{2}\right) \Gamma_{\hat{e}_{i}^{*}, \hat{\tilde{e}}_{j}, \tilde{e}_{s_{1}}^{*}, \tilde{e}_{s_{1}}} \\
& -3 \sum_{s_{1}=1}^{6} A_{0}\left(m_{\tilde{u}_{s_{1}}}^{2}\right) \Gamma_{\hat{\tilde{e}}_{i}^{*}, \hat{\tilde{e}}_{j}, \tilde{u}_{s_{1}}^{*}, \tilde{u}_{s_{1}}}+\sum_{s_{2}=1}^{3} \Gamma_{\hat{e}_{j}^{*}, W-, \tilde{\nu}_{s_{2}}}^{*} \Gamma_{\hat{\tilde{e}}_{i}^{*}, W^{-}, \tilde{\nu}_{s_{2}}} F_{0}\left(p^{2}, m_{\tilde{\nu}_{s_{2}}}^{2}, m_{W}^{2}\right) \\
& +\sum_{s_{2}=1}^{6} \Gamma_{\tilde{e}_{j}^{*}, \gamma, \tilde{e}_{s_{2}}}^{*} \Gamma_{\hat{\tilde{e}}_{i}^{*}, \gamma, \tilde{e}_{s_{2}}} F_{0}\left(p^{2}, m_{\tilde{e}_{s_{2}}}^{2}, 0\right) \\
& +\sum_{s_{2}=1}^{6} \Gamma_{\hat{e}_{j}^{*}, Z, \tilde{e}_{s_{2}}}^{*} \Gamma_{\hat{\tilde{e}}_{i}^{*}, Z, \tilde{e}_{s_{2}}} F_{0}\left(p^{2}, m_{\tilde{e}_{s_{2}}}^{2}, m_{Z}^{2}\right)
\end{aligned}
$$




\section{E.8 Self-energy of sneutrinos}

$$
\begin{aligned}
& \Pi_{\tilde{\nu}_{i}, \tilde{\nu}_{j}}\left(p^{2}\right)=2 A_{0}\left(m_{Z}^{2}\right) \Gamma_{\hat{\tilde{\nu}}_{i}^{*}, \hat{\nu}_{j}, Z, Z}+4 A_{0}\left(m_{W}^{2}\right) \Gamma_{\hat{\tilde{\nu}}_{i}^{*}, \hat{\nu}_{j}, W^{+}, W^{-}} \\
& -2 \sum_{s_{1}=1}^{2} m_{\tilde{\chi}_{s_{1}}^{+}} \sum_{s_{2}=1}^{3}\left[B _ { 0 } ( p ^ { 2 } , m _ { \tilde { \chi } _ { s _ { 1 } } ^ { + } } ^ { 2 } , m _ { e _ { s _ { 2 } } } ^ { 2 } ) m _ { e _ { s _ { 2 } } } \left(\Gamma_{\hat{\tilde{\nu}}_{j}^{*}, \tilde{\chi}_{s_{1}}^{+}, e_{s_{2}}}^{L *} \Gamma_{\hat{\tilde{\nu}}_{i}^{*}, \tilde{\chi}_{s_{1}}^{+}, e_{s_{2}}}^{R}\right.\right. \\
& \left.\left.+\Gamma_{\hat{\tilde{\nu}}_{j}^{*}, \tilde{\chi}_{s_{1}}^{+}, e_{s_{2}}}^{R *} \Gamma_{\hat{\tilde{\nu}}_{i}^{*}, \tilde{\chi}_{s_{1}}^{+}, e_{s_{2}}}^{L}\right)\right] \\
& +\sum_{s_{1}=1}^{2} \sum_{s_{2}=1}^{3}\left[G _ { 0 } ( p ^ { 2 } , m _ { \tilde { \chi } _ { s _ { 1 } } ^ { + } } ^ { 2 } , m _ { e _ { s _ { 2 } } } ^ { 2 } ) \left(\Gamma_{\tilde{\hat{\nu}}_{j}^{*}, \tilde{\chi}_{s_{1}}^{+}, e_{s_{2}}}^{L *} \Gamma_{\tilde{\nu}_{i}^{*}, \tilde{\chi}_{s_{1}}^{+}, e_{s_{2}}}^{L}\right.\right. \\
& \left.\left.+\Gamma_{\tilde{\tilde{\nu}}_{j}^{*}, \tilde{\chi}_{s_{1}}^{+}, e_{s_{2}}}^{R *} \Gamma_{\tilde{\hat{\nu}}_{i}^{*}, \tilde{\chi}_{s_{1}}^{+}, e_{s_{2}}}^{R}\right)\right] \\
& +\sum_{s_{1}=1}^{2} \sum_{s_{2}=1}^{6} B_{0}\left(p^{2}, m_{H_{s_{1}}^{+}}^{2}, m_{\tilde{e}_{s_{2}}}^{2}\right) \Gamma_{\hat{\nu}_{j}^{*}, H_{s_{1}}^{+}, \tilde{e}_{s_{2}}}^{*} \Gamma_{\hat{\tilde{\nu}}_{i}^{*}, H_{s_{1}}^{+}, \tilde{e}_{s_{2}}} \\
& -\frac{1}{2} \sum_{s_{1}=1}^{3} A_{0}\left(m_{A_{s_{1}}^{0}}^{2}\right) \Gamma_{\hat{\tilde{\nu}}_{i}^{*}, \hat{\tilde{\nu}}_{j}, A_{s_{1}}^{0}, A_{s_{1}}^{0}}-\sum_{s_{1}=1}^{3} A_{0}\left(m_{\tilde{\nu}_{s_{1}}}^{2}\right) \Gamma_{\hat{\tilde{\nu}}_{i}^{*}, \hat{\nu}_{j}, \tilde{\nu}_{s_{1}}^{*}, \tilde{\nu}_{s_{1}}} \\
& -\frac{1}{2} \sum_{s_{1}=1}^{3} A_{0}\left(m_{h_{s_{1}}}^{2}\right) \Gamma_{\hat{\tilde{\nu}}_{i}^{*}, \hat{\tilde{\nu}}_{j}, h_{s_{1}}, h_{s_{1}}}-\sum_{s_{1}=1}^{2} A_{0}\left(m_{H_{s_{1}}^{+}}^{2}\right) \Gamma_{\hat{\tilde{\nu}}_{i}^{*}, \hat{\tilde{\nu}}_{j}, H_{s_{1}}^{+}, H_{s_{1}}^{-}} \\
& +\sum_{s_{1}=1}^{3} \sum_{s_{2}=1}^{3} B_{0}\left(p^{2}, m_{h_{s_{1}}}^{2}, m_{\tilde{\nu}_{s_{2}}}^{2}\right) \Gamma_{\hat{\bar{\nu}}_{j}^{*}, h_{s_{1}}, \tilde{\nu}_{s_{2}}}^{*} \Gamma_{\hat{\tilde{\nu}}_{i}^{*}, h_{s_{1}}, \tilde{\nu}_{s_{2}}} \\
& -2 \sum_{s_{1}=1}^{5} m_{\tilde{\chi}_{s_{1}}^{0}} \sum_{s_{2}=1}^{3}\left[B _ { 0 } ( p ^ { 2 } , m _ { \tilde { \chi } _ { s _ { 1 } } ^ { 0 } } ^ { 2 } , 0 ) m _ { \nu _ { s _ { 2 } } } \left(\Gamma_{\hat{\tilde{\nu}}_{j}^{*}, \tilde{\chi}_{s_{1}}^{0}, \nu_{s_{2}}}^{L *} \Gamma_{\hat{\nu}_{i}^{*}, \tilde{\chi}_{s_{1}}^{0}, \nu_{s_{2}}}^{R}\right.\right. \\
& \left.\left.+\Gamma_{\hat{\bar{\nu}}_{j}^{*}, \tilde{\chi}_{s_{1}}^{0}, \nu_{s_{2}}}^{R *} \Gamma_{\tilde{\hat{\nu}}_{i}^{*}, \tilde{\chi}_{s_{1}}^{0}, \nu_{s_{2}}}^{L}\right)\right] \\
& +\sum_{s_{1}=1}^{5} \sum_{s_{2}=1}^{3}\left[G _ { 0 } ( p ^ { 2 } , m _ { \tilde { \chi } _ { s _ { 1 } } ^ { 0 } } ^ { 2 } , 0 ) \left(\Gamma_{\hat{\tilde{\nu}}_{j}^{*}, \tilde{\chi}_{s_{1}}^{0}, \nu_{s_{2}}}^{L *} \Gamma_{\hat{\tilde{\nu}}_{i}^{*}, \tilde{\chi}_{s_{1}}^{0}, \nu_{s_{2}}}^{L}\right.\right. \\
& \left.\left.+\Gamma_{\tilde{\tilde{\nu}}_{j}^{*}, \tilde{\chi}_{s_{1}}^{0}, \nu_{s_{2}}}^{R *} \Gamma_{\tilde{\hat{\nu}}_{i}^{*}, \tilde{\chi}_{s_{1}}^{0}, \nu_{s_{2}}}^{R}\right)\right] \\
& -3 \sum_{s_{1}=1}^{6} A_{0}\left(m_{\tilde{d}_{s_{1}}}^{2}\right) \Gamma_{\hat{\tilde{\nu}}_{i}^{*}, \hat{\nu}_{j}, \tilde{d}_{s_{1}}^{*}, \tilde{d}_{s_{1}}}-\sum_{s_{1}=1}^{6} A_{0}\left(m_{\tilde{e}_{s_{1}}}^{2}\right) \Gamma_{\hat{\tilde{\nu}}_{i}^{*}, \hat{\nu}_{j}, \tilde{e}_{s_{1}}^{*}, \tilde{e}_{s_{1}}} \\
& -3 \sum_{s_{1}=1}^{6} A_{0}\left(m_{\tilde{u}_{s_{1}}}^{2}\right) \Gamma_{\hat{\tilde{\nu}}_{i}^{*}, \hat{\nu}_{j}, \tilde{u}_{s_{1}}^{*}, \tilde{u}_{s_{1}}}+\sum_{s_{2}=1}^{3} \Gamma_{\hat{\nu}_{j}^{*}, Z, \tilde{\nu}_{s_{2}}}^{*} \Gamma_{\hat{\tilde{\nu}}_{i}^{*}, Z, \tilde{\nu}_{s_{2}}} F_{0}\left(p^{2}, m_{\tilde{\nu}_{s_{2}}}^{2}, m_{Z}^{2}\right) \\
& +\sum_{s_{2}=1}^{6} \Gamma_{\hat{\nu}_{j}^{*}, W^{+}, \tilde{e}_{s_{2}}}^{*} \Gamma_{\hat{\nu}_{i}^{*}, W^{+}, \tilde{e}_{s_{2}}} F_{0}\left(p^{2}, m_{\tilde{e}_{s_{2}}}^{2}, m_{W}^{2}\right)
\end{aligned}
$$

Open Access. This article is distributed under the terms of the Creative Commons Attribution Noncommercial License which permits any noncommercial use, distribution, and reproduction in any medium, provided the original author(s) and source are credited. 


\section{References}

[1] J. Wess and B. Zumino, Supergauge Transformations In Four-Dimensions, Nucl. Phys. B70 (1974) 39 [SPIRES].

[2] P. Fayet and S. Ferrara, Supersymmetry, Phys. Rept. 32 (1977) 249 [SPIRES].

[3] H.P. Nilles, Supersymmetry, Supergravity and Particle Physics, Phys. Rept. 110 (1984) 1 [SPIRES].

[4] H.E. Haber and G.L. Kane, The Search for Supersymmetry: Probing Physics Beyond the Standard Model, Phys. Rept. 117 (1985) 75 [SPIRES].

[5] E. Witten, Dynamical Breaking of Supersymmetry, Nucl. Phys. B 188 (1981) 513 [SPIRES].

[6] S. Dimopoulos, S. Raby and F. Wilczek, Supersymmetry and the Scale of Unification, Phys. Rev. D 24 (1981) 1681 [SPIRES].

[7] L.E. Ibáñez and G.G. Ross, Low-Energy Predictions in Supersymmetric Grand Unified Theories, Phys. Lett. B 105 (1981) 439 [SPIRES].

[8] U. Amaldi, W. de Boer and H. Furstenau, Comparison of grand unified theories with electroweak and strong coupling constants measured at LEP, Phys. Lett. B 260 (1991) 447 [SPIRES].

[9] P. Langacker and M.-x. Luo, Implications of precision electroweak experiments for $M_{t}, \rho_{0}$, $\sin ^{2} \theta_{W}$ and grand unification, Phys. Rev. D 44 (1991) 817 [SPIRES].

[10] J.R. Ellis, S. Kelley and D.V. Nanopoulos, Probing the desert using gauge coupling unification, Phys. Lett. B 260 (1991) 131 [SPIRES].

[11] J.R. Ellis, J.S. Hagelin, D.V. Nanopoulos, K.A. Olive and M. Srednicki, Supersymmetric relics from the big bang, Nucl. Phys. B 238 (1984) 453 [SPIRES].

[12] F.D. Steffen, Dark Matter Candidates - Axions, Neutralinos, Gravitinos and Axinos, Eur. Phys. J. C 59 (2009) 557 [arXiv:0811.3347] [SPIRES].

[13] J.E. Kim and H.P. Nilles, The $\mu$ Problem and the Strong CP Problem, Phys. Lett. B 138 (1984) 150 [SPIRES].

[14] P. Fayet, Supergauge Invariant Extension of the Higgs Mechanism and a Model for the electron and Its Neutrino, Nucl. Phys. B 90 (1975) 104 [SPIRES].

[15] P. Fayet, Supersymmetry and Weak, Electromagnetic and Strong Interactions, Phys. Lett. B 64 (1976) 159 [SPIRES].

[16] P. Fayet, Spontaneously Broken Supersymmetric Theories of Weak, Electromagnetic and Strong Interactions, Phys. Lett. B 69 (1977) 489 [SPIRES].

[17] P. Fayet, Relations Between the Masses of the Superpartners of Leptons and Quarks, the Goldstino Couplings and the Neutral Currents, Phys. Lett. B 84 (1979) 416 [SPIRES].

[18] H.P. Nilles, M. Srednicki and D. Wyler, Weak Interaction Breakdown Induced by Supergravity, Phys. Lett. B 120 (1983) 346 [SPIRES].

[19] J.M. Frere, D.R.T. Jones and S. Raby, Fermion Masses and Induction of the Weak Scale by Supergravity, Nucl. Phys. B 222 (1983) 11 [SPIRES].

[20] J.P. Derendinger and C.A. Savoy, Quantum Effects and SU(2) $\times \mathrm{U}(1)$ Breaking in Supergravity Gauge Theories, Nucl. Phys. B 237 (1984) 307 [SPIRES]. 
[21] J.R. Ellis, J.F. Gunion, H.E. Haber, L. Roszkowski and F. Zwirner, Higgs Bosons in a Nonminimal Supersymmetric Model, Phys. Rev. D 39 (1989) 844 [SPIRES].

[22] M. Drees, Supersymmetric Models with Extended Higgs Sector, Int. J. Mod. Phys. A 4 (1989) 3635 [SPIRES].

[23] U. Ellwanger, M. Rausch de Traubenberg and C.A. Savoy, Particle spectrum in supersymmetric models with a gauge singlet, Phys. Lett. B 315 (1993) 331 [hep-ph/9307322] [SPIRES].

[24] U. Ellwanger, M. Rausch de Traubenberg and C.A. Savoy, Higgs phenomenology of the supersymmetric model with a gauge singlet, Z. Phys. C 67 (1995) 665 [hep-ph/9502206] [SPIRES].

[25] U. Ellwanger, M. Rausch de Traubenberg and C.A. Savoy, Phenomenology of supersymmetric models with a singlet, Nucl. Phys. B 492 (1997) 21 [hep-ph/9611251] [SPIRES].

[26] T. Elliott, S.F. King and P.L. White, Unification constraints in the next-to-minimal supersymmetric standard model, Phys. Lett. B 351 (1995) 213 [hep-ph/9406303] [SPIRES].

[27] S.F. King and P.L. White, Resolving the constrained minimal and next-to-minimal supersymmetric standard models, Phys. Rev. D 52 (1995) 4183 [hep-ph/9505326] [SPIRES].

[28] G. Bélanger, F. Boudjema, C. Hugonie, A. Pukhov and A. Semenov, Relic density of dark matter in the NMSSM, JCAP 09 (2005) 001 [hep-ph/0505142] [SPIRES].

[29] C. Hugonie, G. Bélanger and A. Pukhov, Dark Matter in the Constrained NMSSM, JCAP 11 (2007) 009 [arXiv:0707.0628] [SPIRES].

[30] U. Ellwanger and C. Hugonie, NMSPEC: A Fortran code for the sparticle and Higgs masses in the NMSSM with GUT scale boundary conditions, Comput. Phys. Commun. 177 (2007) 399 [hep-ph/0612134] [SPIRES].

[31] U. Ellwanger and C. Hugonie, NMHDECAY 2.0: An Updated program for sparticle masses, Higgs masses, couplings and decay widths in the NMSSM, Comput. Phys. Commun. 175 (2006) 290 [hep-ph/0508022] [SPIRES].

[32] U. Ellwanger, J.F. Gunion and C. Hugonie, NMHDECAY: A Fortran code for the Higgs masses, couplings and decay widths in the NMSSM, JHEP 02 (2005) 066 [hep-ph/0406215] [SPIRES].

[33] G. Degrassi and P. Slavich, On the radiative corrections to the neutral Higgs boson masses in the NMSSM, Nucl. Phys. B $\mathbf{8 2 5}$ (2010) 119 [arXiv:0907.4682] [SPIRES].

[34] D.M. Pierce, J.A. Bagger, K.T. Matchev and R.-j. Zhang, Precision corrections in the minimal supersymmetric standard model, Nucl. Phys. B 491 (1997) 3 [hep-ph/9606211] [SPIRES].

[35] U. Ellwanger, C. Hugonie and A.M. Teixeira, The Next-to-Minimal Supersymmetric Standard Model, arXiv:0910.1785 [SPIRES].

[36] F. Staub, Automatic Calculation of supersymmetric Renormalization Group Equations and Self Energies, arXiv:1002.0840 [SPIRES].

[37] F. Staub, From Superpotential to Model Files for FeynArts and CalcHep/CompHEP, Comput. Phys. Commun. 181 (2010) 1077 [arXiv:0909. 2863] [SPIRES].

[38] F. Staub, SARAH, arXiv:0806.0538 [SPIRES]. 
[39] A.H. Chamseddine, R.L. Arnowitt and P. Nath, Locally Supersymmetric Grand Unification, Phys. Rev. Lett. 49 (1982) 970 [SPIRES].

[40] A. Djouadi et al., Benchmark scenarios for the NMSSM, JHEP 07 (2008) 002 [arXiv: 0801.4321] [SPIRES].

[41] A. Djouadi, U. Ellwanger and A.M. Teixeira, Phenomenology of the constrained NMSSM, JHEP 04 (2009) 031 [arXiv:0811.2699] [SPIRES].

[42] S.P. Martin and M.T. Vaughn, Two loop renormalization group equations for soft supersymmetry breaking couplings, Phys. Rev. D 50 (1994) 2282 [Erratum ibid. D 78 (2008) 039903] [hep-ph/9311340] [SPIRES].

[43] F. Staub, B. Herrmann and W. Porod, Analytical expressions of the Next-to-minimal Supersymmetric Standard Model, www.physik.uni-wuerzburg.de/ fnstaub/NMSSM.pdf.

[44] W. Porod, SPheno, a program for calculating supersymmetric spectra, SUSY particle decays and SUSY particle production at $e^{+} e^{-}$colliders, Comput. Phys. Commun. 153 (2003) 275 [hep-ph/0301101] [SPIRES].

[45] B.C. Allanach et al., SUSY Les Houches Accord 2, Comp. Phys. Commun. 180 (2009) 8 [arXiv:0801.0045] [SPIRES].

[46] G. Bélanger, S. Kraml and A. Pukhov, Comparison of SUSY spectrum calculations and impact on the relic density constraints from WMAP, Phys. Rev. D 72 (2005) 015003 [hep-ph/0502079] [SPIRES].

[47] E. Komatsu et al., Seven-Year Wilkinson Microwave Anisotropy Probe (WMAP) Observations: Cosmological Interpretation, arXiv:1001.4538 [SPIRES].

[48] G. Bélanger, F. Boudjema, A. Pukhov and A. Semenov, MicrOMEGAs2.0: A program to calculate the relic density of dark matter in a generic model, Comput. Phys. Commun. 176 (2007) 367 [hep-ph/0607059] [SPIRES].

[49] A. Pukhov, Calchep 2.3: MSSM, structure functions, event generation, 1 and generation of matrix elements for other packages, hep-ph/0412191 [SPIRES].

[50] B. Herrmann and M. Klasen, SUSY-QCD Corrections to Dark Matter Annihilation in the Higgs Funnel, Phys. Rev. D 76 (2007) 117704 [arXiv: 0709.0043] [SPIRES];

[51] B. Herrmann, M. Klasen and K. Kovarik, Neutralino Annihilation into Massive Quarks with SUSY-QCD Corrections, Phys. Rev. D 79 (2009) 061701 [arXiv:0901.0481] [SPIRES].

[52] B. Herrmann, M. Klasen and K. Kovarik, SUSY-QCD effects on neutralino dark matter annihilation beyond scalar or gaugino mass unification, Phys. Rev. D 80 (2009) 085025 [arXiv:0907.0030] [SPIRES]. 